\title{
A $Z$-set unknotting theorem for Nöbeling spaces
}

by

\author{
Michael Levin (Be'er Sheva)
}

\begin{abstract}
We prove a $Z$-set unknotting theorem for Nöbeling spaces. The theorem is proved for a certain model of Nöbeling spaces.
\end{abstract}

1. Introduction. All spaces are assumed to be separable metrizable. A manifold means a manifold with (possibly empty) boundary, and a triangulated space means a locally finite simplicial complex which we identify with the underlying space. For a triangulated space we consider only triangulations compatible with the PL-structure of the space. All triangulated manifolds are assumed to be combinatorial.

A complete $n$-dimensional metric space $X$ is said to be an $n$-dimensional Nöbeling space if the following conditions are satisfied:

(i) $X$ is an absolute extensor in dimension $n$, that is, every map $f: A \rightarrow$ $X$ from a closed subset $A$ of a space $Y$ of dimension $\leq n$ extends over $Y$;

(ii) every map $f: Y \rightarrow X$ from a complete metric space $Y$ of dimension $\leq n$ can be arbitrarily closely approximated by a closed embedding, that is, for every open cover $\mathcal{U}$ of $X$ there is a closed embedding $g: Y \rightarrow X$ which is $\mathcal{U}$-close to $f(\mathcal{U}$-close means that for every $y \in Y$ there is an element of $\mathcal{U}$ that contains both $f(y)$ and $g(y))$.

Examples of Nöbeling spaces can be constructed as follows.

Let us say that a point of a triangulated space is rational if it has rational barycentric coordinates with respect to the triangulation of the space. By a rational map $f: M_{1} \rightarrow M_{2}$ between triangulated spaces $M_{1}$ and $M_{2}$ with the triangulations $\mathcal{T}_{1}$ and $\mathcal{T}_{2}$ respectively we mean a PL-map that sends the rational points of $M_{1}$ to the rational points of $M_{2}$. Note that if $f$ is a rational embedding and $f(x)$ is a rational point in $M_{2}$ then $x$ is rational in $M_{1}$.

2000 Mathematics Subject Classification: 55M10, 54F45.

Key words and phrases: $Z$-set unknotting theorem, Nöbeling spaces.

The author was supported by ISF grant 836/08. 
Indeed, let $\Delta_{1}$ be a simplex of $\mathcal{T}_{1}$ containing $x$. Since $f$ is a PL-embedding there are a simplex $\Delta_{1}^{\prime}$ linearly embedded into $\Delta_{1}$ and a simplex $\Delta_{2} \in \mathcal{T}_{2}$ such that $x \in \Delta_{1}^{\prime}, \operatorname{dim} \Delta_{1}^{\prime}=\operatorname{dim} \Delta_{1}$ and $f$ linearly sends $\Delta_{1}^{\prime}$ into $\Delta_{2}$. Then the rational points of $M_{1}$ contain a subset which is dense in $\Delta_{1}^{\prime}$ and therefore we can choose points $x_{0}, \ldots, x_{n} \in \Delta_{1}^{\prime}, n=\operatorname{dim} \Delta_{1}^{\prime}$, which are rational in $M_{1}$ and in general position in $\Delta_{1}^{\prime}$. Since $f$ is rational and linear on $\Delta_{1}^{\prime}$, we see that $f\left(x_{0}\right), \ldots, f\left(x_{n}\right)$ are rational in $M_{2}$ and in general position in $\Delta_{2}^{\prime}=f\left(\Delta_{1}^{\prime}\right)$. Hence, since $f(x)$ is rational in $M_{2}, f(x)$ can be represented as $f(x)=\lambda_{0} f\left(x_{0}\right)+\cdots+\lambda_{n} f\left(x_{n}\right), \lambda_{0}+\cdots+\lambda_{n}=1$, with $\lambda_{0}, \ldots, \lambda_{n}$ (not necessarily non-negative) rational numbers. Then $x=\lambda_{0} x_{0}+\cdots+\lambda_{n} x_{n}$ and hence $x$ is rational in $M_{1}$.

Two triangulations of a space are said to be rationally equivalent if the identity map is a rational map with respect to these triangulations. Let $M$ be a triangulated space. Every triangulation of $M$ which is rationally equivalent to the given triangulation of $M$ is said to be a rational triangulation, and the class of all rational triangulations is said to be the rational structure of $M$. Denote by $M(k)$ the subspace of $M$ which is the complement of the union of all the triangulated spaces of dimension $\leq k$ which are rationally embedded in $M$.

The space $M(k)$ admits the following interpretation. Let $H$ be a Hilbert space. A point in $H$ is said to be rational if it has rational coordinates and only finitely many of them are non-zero. A $k$-dimensional plane in $H$ is said to be rational if it is spanned by $k+1$ rational points. Fix a rational triangulation of $M$ and embed $M$ in $H$ by an embedding which sends the vertices to rational points and which is linear on every simplex of the triangulation. Denote by $K$ the union of all rational $k$-dimensional planes in $H$. Then $M(k)=M \backslash K$. Indeed, for every simplex $\Delta$ of $M$ and every rational $k$-dimensional plane $L, \Delta \cap L$ admits a triangulation for which it is rationally embedded in $M$ and therefore $M(k) \subset M \backslash K$. Now let $e: \Delta^{\prime} \rightarrow M$ be a rational embedding of a simplex $\Delta^{\prime}$ of dimension $\leq k$. Then there is a (not necessarily rational) triangulation $\mathcal{T}$ of $\Delta^{\prime}$ such that $e$ is linear on every simplex of $\mathcal{T}$. Since every $\Delta^{\prime \prime} \in \mathcal{T}$ with $\operatorname{dim} \Delta^{\prime \prime}=\operatorname{dim} \Delta^{\prime}$ has a dense subset of points with rational barycentric coordinates with respect to $\Delta^{\prime}$, we conclude that $e\left(\Delta^{\prime \prime}\right)$ is contained in a $k$-dimensional rational plane in $H$. Thus $e\left(\Delta^{\prime}\right) \subset K$ and hence $M(k)=M \backslash K$.

Let us state the following important fact, leaving its proof to the reader.

TheOREM 1.1. Let $M$ be a triangulated $m$-dimensional manifold, let $k \geq 0$ be an integer and let $n=m-k-1$. If $M$ is $(n-1)$-connected and $m \geq 2 n+1$ then $M(k)$ is an $n$-dimensional Nöbeling space.

A space $M(k)$ satisfying the assumptions of Theorem 1.1 will be called a Nöbeling space modeled on a triangulated manifold. 
A subset $A$ of a space $X$ is called a $Z$-set if $A$ is closed in $X$ and the identity map of $X$ can be arbitrarily closely approximated by a map $f: X \rightarrow X$ with $f(X) \cap A=\emptyset$. Note that if $X$ is an $n$-dimensional Nöbeling space modeled on a manifold $M$ and $A \subset X$ is a $Z$-set in $X$ then $X \backslash A$ is also an $n$-dimensional Nöbeling space modeled on the manifold $N=M \backslash$ the closure of $A$ in $M$ (the rational structure of $N$ is defined such that the inclusion is a rational map; see the beginning of Section 3 for details).

The main result of this paper is the following $Z$-set unknotting theorem.

Theorem 1.2. Let $X_{1}$ and $X_{2}$ be $n$-dimensional Nöbeling spaces and let $A_{1}$ and $A_{2}$ be $Z$-sets in $X_{1}$ and $X_{2}$ respectively such that $X_{1} \backslash A_{1}$ and $X_{2} \backslash A_{2}$ are homeomorphic to $n$-dimensional Nöbeling spaces modeled on triangulated manifolds. If $A_{1}$ and $A_{2}$ are homeomorphic then any homeomorphism between $A_{1}$ and $A_{2}$ can be extended to a homeomorphism between $X_{1}$ and $X_{2}$.

In fact, we will prove a slightly stronger version of Theorem 1.2 which is presented in Theorem 3.1. $Z$-set unknotting theorems similar to Theorem 1.2 were also proved using different approaches by A. Nagórko [9] and for a restricted class of $Z$-sets by S. Ageev [1], [2], [3]. The proof of Theorem 1.2 is self-contained and relies only on well-known facts of PL-topology [6], [11], Nöbeling spaces [5] and elementary properties of partitions presented at the very beginning of [4]. Some ideas of the proof of Theorem 1.2 came from [7].

The results of this paper along with some additional arguments apply to validate the characterization theorem for Nöbeling saying that any two Nöbeling spaces of the same dimension are homeomorphic (see [8]). The characterization theorem implies that the assumptions on the complements $X_{1} \backslash A_{1}$ and $X_{2} \backslash A_{2}$ in Theorem 1.2 are automatically satisfied and therefore can be dropped.

The characterization theorem for Nöbeling spaces was also proved by Nagórko [9] and Ageev [1], [2], [3]. Note that both a $Z$-set unknotting theorem and the characterization theorem were proved by Nagórko in a more general setting of Nöbeling manifolds.

I would like to thank the referee of this paper for valuable remarks and a simplification in Section 3.

\section{Preliminaries}

2.1. General. Let $M$ be a triangulated manifold. The geometric interior Int $M$ of $M$ is the set of points having a neighborhood PL-homeomorphic to an Euclidean space of $\operatorname{dimension} \operatorname{dim} M$. The set $\partial M=M \backslash \operatorname{Int} M$ is the geometric boundary of $M$. 
A triangulated space (manifold) PL-embedded in $M$ is said to be a $P L$ subspace ( $P L$-submanifold) of $M$. An open subset of a triangulated space is always considered with the induced PL-structure for which the inclusion is a PL-map. Note that an open subset of a PL-submanifold of $M$ is also a PL-submanifold of $M$.

A subset $P$ of $M$ is said to be a $P L$-subcomplex of $M$ if there is a triangulation of $M$ for which $P$ is a subcomplex. A subset $R$ of $M$ is said to be a $P L$-presented subset of $M$ if there are closed subsets $R_{1} \subset \cdots \subset R_{n}$ of $M$ such that $R_{n}=R, R_{1}$ is a PL-subcomplex of $M$ and $R_{i+1} \backslash R_{i}$ is a PL-subcomplex of $M \backslash R_{i}, i=1, \ldots, n-1$.

A collection $\mathcal{P}$ of subsets of $M$ is said to be a decomposition of $M$ if $\mathcal{P}$ is a locally finite cover of $M$ and the elements of $\mathcal{P}$ are PL-subcomplexes of $M$. By a finite intersection of a decomposition $\mathcal{P}$ we mean an intersection of finitely many elements of $\mathcal{P}$ (the elements of $\mathcal{P}$ are also considered as finite intersections). Note that since $\mathcal{P}$ is locally finite, any non-empty intersection of elements of $\mathcal{P}$ must be a finite intersection of $\mathcal{P}$. It is clear that for a PLsubmanifold $N$ of $M$, the restriction $\mathcal{P} \mid N=\{P \cap N: P \in \mathcal{P}\}$ of $\mathcal{P}$ to $N$ is a decomposition of $N$.

A decomposition $\mathcal{P}$ of $M$ is said to be a partition of $M$ if each finite intersection of $\mathcal{P}$ is a PL-manifold, the geometric interiors of any two non-equal finite intersections are disjoint and for every non-empty finite intersection $P=P_{0} \cap \cdots \cap P_{t}$ of distinct elements $P_{0}, \ldots, P_{t} \in \mathcal{P}, \operatorname{dim} P=\operatorname{dim} M-t$.

A decomposition $\mathcal{P}$ of $M$ is said to be a partition on a $P L$-submanifold $N$ of $M$ if $\mathcal{P} \mid N$ is a partition of $N$ (in this case we also say that $\mathcal{P}$ forms a partition on $N$ or $\mathcal{P}$ restricted to $N$ is a partition).

The PL-notions defined above can be translated to the corresponding rational notions by referring to the rational structure of $M$ instead of the PL-structure (see Section 1). Thus $P \subset M$ is a rational subspace if there is a triangulation of $P$ for which the inclusion is a rational map, $P$ is a rational subcomplex if there is a rational triangulation of $M$ for which $P$ is a subcomplex, $\mathcal{P}$ is a rational decomposition of $M$ if the elements of $\mathcal{P}$ are rational subcomplexes etc. Note that an open subset of $M$ is a rational subset of $M$ and it is always considered with the rational structure for which the inclusion is a rational map. Thus the rationally presented sets are well-defined as well.

A subset $P$ of $M$ is $q$-connected, $q \geq 0$, if $\pi_{i}(P)$ is trivial for every $0 \leq i \leq q$. We will say that $P$ is $l$-co-connected, $l \geq 0$, if $P$ is $(\operatorname{dim} P-l)$ connected (we assume that $P$ is $q$-connected for every $q<0$ and $P$ is $l$-coconnected for every $l>\operatorname{dim} P$ ). A partition $\mathcal{P}$ is said to be $l$-co-connected if every finite intersection of $\mathcal{P}$ is $l$-co-connected.

A map $f: X \rightarrow M$ is said to be in general position with a triangulation of $M$ if, for every simplex $\Delta$ of the triangulation, $\operatorname{dim} f^{-1}(\Delta) \leq$ 
$\operatorname{dim} X+\operatorname{dim} \Delta-\operatorname{dim} M$. Every map from $X$ to $M$ can be arbitrarily closely approximated by a general position map. Moreover, let $F$ be closed in $X$ and a map $f: X \rightarrow M$ restricted to $F$ be in general position. Then $f$ can be arbitrarily closely approximated by a general position map $g: X \rightarrow M$ such that $g$ coincides with $f$ on $F$ and $g(X \backslash F) \subset \operatorname{Int} M$. A map $f: X \rightarrow M$ is said to be in general position with a decomposition of $M$ if it is in general position with a triangulation underlying this decomposition (a triangulation of $M$ for which the elements of the decomposition are subcomplexes).

An $n$-dimensional cube $B^{n}$ is a set of the form $B^{n}=\left\{\left(x_{1}, \ldots, x_{n}\right)\right.$ : $\left.-r_{i} \leq x_{i} \leq r_{i}, r_{i}>0, i=1, \ldots, n\right\}$ in the Euclidean space $\mathbb{R}^{n}$. Thus we always assume that the origin $O$ is at the center of $B^{n}$. Considering the product $B^{n} \times B^{m}$ of two cubes we identify $B^{n}$ and $B^{m}$ with $B^{n} \times O$ and $O \times B^{m}$ respectively.

Let $\mathcal{A}$ and $\mathcal{B}$ be collections of subsets of a set $X$ and let $C \subset X$. We define $\operatorname{st}(C, \mathcal{A})=\bigcup\{A: A \in \mathcal{A}, A \cap C \neq \emptyset\}, \operatorname{st}(\mathcal{A}, \mathcal{B})=\{\operatorname{st}(A, \mathcal{B}): A \in \mathcal{A}\}$, st $\mathcal{A}=\operatorname{st}^{1} \mathcal{A}=\operatorname{st}(\mathcal{A}, \mathcal{A})$ and by induction $\left.\mathrm{st}^{i+1} \mathcal{A}=\operatorname{st}^{i t^{i}} \mathcal{A}\right)$.

2.2. Elementary properties of partitions. If $\mathcal{P}$ is a partition of a triangulated manifold $M$ then $\mathcal{P} \mid V$ is a partition of $V$ for every open $V \subset M$. Let $\mathcal{P}$ be a decomposition of a triangulated manifold $M$ and let $\mathcal{V}$ be an open cover $M$ such that $\mathcal{P} \mid V$ is a partition of $V$ for every $V \in \mathcal{V}$. Then $\mathcal{P}$ is a partition of $M$.

Assume that $M$ is a triangulated space. One can show that $M \times(0,1)$ or $M \times[0,1)$ is a PL-manifold if and only if $M$ is a PL-manifold. This implies that for a triangulated manifold $N$, the product $M \times N$ is a PLmanifold if and only if $M$ is a PL-manifold. Thus if $M$ and $N$ are triangulated manifolds and $\mathcal{P}$ is a decomposition of $M$, then the decomposition $\mathcal{P} \times N=\{P \times N: P \in \mathcal{P}\}$ is a partition of $M \times N$ if and only if $\mathcal{P}$ is a partition of $M$.

The following properties are proved in [4] for compact manifolds, but their proof also applies for the non-compact case.

Let $\mathcal{P}$ be a partition of a triangulated manifold $M$, let $\mathcal{P}=\bigcup\left\{\mathcal{P}_{i}\right.$ : $i=1,2, \ldots\}$ be a splitting of $\mathcal{P}$ into disjoint subfamilies $\mathcal{P}_{i}$ and let $Q_{i}=$ $\bigcup\left\{P: P \in \mathcal{P}_{i}\right\}$. Then $\mathcal{Q}=\left\{Q_{1}, Q_{2}, \ldots\right\}$ is a partition of $M$ (see 1.1.5 of [4]). In particular, any union of elements of a partition is a PL-manifold.

Let $\mathcal{P}$ be a partition of a triangulated manifold $M$. Then for every finite intersection $P$ of $\mathcal{P}, P \cap \partial M \subset \partial P$ (see 1.1.9 of [4]).

Let $M$ be an $m$-dimensional triangulated manifold, $\mathcal{P}$ a partition of $M$ and $\mathcal{Q}$ a decomposition of $M$ such that for every finite intersection $P$ of $\mathcal{P}, \mathcal{Q} \mid P$ is a partition of $P$. Then $\mathcal{Q}$ is a partition of $M$ (see 1.1.11 of [4]). In particular, if $M=M_{1} \cup M_{2}$ is a decomposition of $M$ into two $m$-dimensional PL-submanifolds $M_{1}$ and $M_{2}$ such that $N=M_{1} \cap M_{2}$ is 
an $(m-1)$-dimensional PL-submanifold of both $\partial M_{1}$ and $\partial M_{2}$, and $\mathcal{Q}$ is a decomposition of $M$ such that $\mathcal{Q}\left|M_{1}, \mathcal{Q}\right| M_{2}$ and $\mathcal{Q} \mid N$ are partitions, then $\mathcal{Q}$ is a partition of $M$.

2.3. A matching of partitions. Let $\mathcal{P}_{1}$ and $\mathcal{P}_{2}$ be partitions of triangulated manifolds $M_{1}$ and $M_{2}$ respectively. A one-to-one correspondence $\mu: \mathcal{P}_{1} \rightarrow \mathcal{P}_{2}$ is said to be a matching of $\mathcal{P}_{1}$ and $\mathcal{P}_{2}$ if it induces a one-to-one correspondence between non-empty intersections. This means that for every finite intersection $P=P_{0} \cap \cdots \cap P_{t}$ of distinct elements $P_{0}, \ldots, P_{t} \in \mathcal{P}_{1}$, $\mu(P)=\mu\left(P_{0}\right) \cap \cdots \cap \mu\left(P_{t}\right)=\emptyset$ if and only if $P=\emptyset$.

Assume that $\mu: \mathcal{P}_{1} \rightarrow \mathcal{P}_{2}$ is a matching of partitions $\mathcal{P}_{1}$ and $\mathcal{P}_{2}$ such that $\mathcal{P}_{2}$ is $l$-co-connected. Let $F$ be a closed subset of $X \operatorname{such}$ that $\operatorname{dim} X \leq$ $m_{i}-l+1, m_{i}=\operatorname{dim} M_{i}, i=1,2$, and let $f_{i}: F \rightarrow M_{i}$ be maps such that $f_{1}^{-1}(P)=f_{2}^{-1}(\mu(P))$ for every finite intersection $P$ of $\mathcal{P}_{1}$. Assume that a map $f_{1}^{\prime}: X \rightarrow M_{1}$ extends $f_{1}$ so that $f_{1}^{\prime}$ is in general position with $\mathcal{P}_{1}$. Then there is a map $f_{2}^{\prime}: X \rightarrow M_{2}$ such that $f_{2}^{\prime}$ extends $f_{2}$ and $\left(f_{1}^{\prime}\right)^{-1}(P)=\left(f_{2}^{\prime}\right)^{-1}(\mu(P))$ for every finite intersection $P$ of $\mathcal{P}_{1}$.

The required extension of $f_{2}$ can be constructed by induction on the codimension $t=m_{2}, m_{2}-1, m_{2}-2, \ldots, 0$ of the intersections of $\mathcal{P}_{2}$. Assume that we have already extended $f_{2}$ to $f_{2}^{\prime}$ on $X_{t}=$ the union of the preimages of all the intersections of $\mathcal{P}_{2}$ of dimension $\leq m_{2}-t$ such that $f_{2}^{\prime}$ on $X_{t}$ has the required properties and let an intersection $P$ of $\mathcal{P}_{2}$ be of dimension $m_{2}-t+1$. Then for $X_{P}=\left(f_{1}^{\prime}\right)^{-1}\left(\mu^{-1}(P)\right), \operatorname{dim} X_{P} \leq \operatorname{dim} X+\operatorname{dim} \mu^{-1}(P)-m_{1}=$ $\operatorname{dim} X+\operatorname{dim} P-m_{2}$. Since $P$ is $(\operatorname{dim} P-l)$-connected and $\operatorname{dim} P-l \geq$ $\operatorname{dim} P-m_{2}+\operatorname{dim} X-1 \geq \operatorname{dim} X_{P}-1, f_{2}^{\prime}$ restricted to $X_{P} \cap X_{t}$ can be extended over $X_{P}$ so that $f_{2}^{\prime}\left(X_{P} \backslash X_{t}\right) \subset \operatorname{Int} P$.

Thus we can extend $f_{2}$ to a map $f_{2}^{\prime}$ with the required properties. This extension will be called a transfer of the extension $f_{1}^{\prime}$ via the matching $\mu$.

Assume that $\mu: \mathcal{P}_{1} \rightarrow \mathcal{P}_{2}$ is a matching of partitions on manifolds $M_{1}$ and $M_{2}$ respectively such that $\mathcal{P}_{i}$ is $l_{i}$-co-connected and $m_{1}-l_{1}=m_{2}-l_{2}$ where $m_{i}=\operatorname{dim} M_{i}$. Let $n \leq m_{1}-l_{1}$ and let $P_{1} \subset P_{1}^{\prime}$ be subsets of $M_{1}$ such that $P_{1}, P_{1}^{\prime}$ are unions of elements of $\mathcal{P}_{1}$ and the inclusion $P_{1} \subset P_{1}^{\prime}$ induces the zero-homomorphism of the homotopy groups in dimensions $\leq n$. Then the inclusion $P_{2}=\mu\left(P_{1}\right) \subset P_{2}^{\prime}=\mu\left(P_{1}^{\prime}\right)$ also induces the zero-homomorphism of the homotopy groups in dimensions $\leq n$. Indeed, take a map $f_{2}: S^{p} \rightarrow P_{2}$, $p \leq n$, from a $p$-dimensional sphere $S^{p}$ into $P_{2}$. Since $P_{2}$ is a manifold we can homotope $f_{2}$ inside $P_{2}$ to a general position map. Thus we assume that $f_{2}$ is a general position map and transfer this map via the matching $\mu$ to $f_{1}: S^{p} \rightarrow P_{1}$. Extend $f_{1}$ to a general position map $f_{1}^{\prime}: B^{p+1} \rightarrow P_{1}^{\prime}$ of a $(p+1)$-dimensional ball $B^{p+1}$ such that $S^{p}=\partial B^{p+1}$ and once again transfer this extension to an extension $f_{2}^{\prime}: B^{p+1} \rightarrow P_{2}^{\prime}$ of $f_{2}$. Thus $f_{2}$ is null-homotopic in $P_{2}^{\prime}$. 
2.4. Improving connectivity of intersections - a summary. Assume that $m \geq 2 q+1$ and $l=m-q+2, M$ is a triangulated $m$-dimensional $(q-1)$ connected $(=(l-1)$-co-connected) manifold and $F$ is a PL-subcomplex of $M$ lying in Int $M$. Let $\mathcal{P}$ be a decomposition of $M$ which forms an $l$-coconnected partition on $U=M \backslash F$ and let $0 \leq t \leq m-l+1$. Suppose that the finite intersections of $\mathcal{P} \mid U$ of dimension $>m-t$ are $(l-1)$-co-connected. We will describe in Section 4 a procedure how to improve the connectivity of the intersections of $\mathcal{P} \mid U$ of dimension $m-t$.

Namely, we will show how to modify $M$ to an open subset $M^{\prime} \subset M$, $F$ to a PL-subcomplex $F^{\prime}$ of $M^{\prime}$ lying in Int $M^{\prime}$ and each $P \in \mathcal{P}$ to a PL-subcomplex $P^{\prime}$ of $M^{\prime}$ so that $R=M \backslash M^{\prime}$ is a PL-presented subset of $M, \operatorname{dim} R \leq q, \operatorname{dim} F^{\prime} \leq \max \{m-q-1, \operatorname{dim} F\}, \mathcal{P}^{\prime}=\left\{P^{\prime}: P \in \mathcal{P}\right\}$ is a decomposition of $M^{\prime}$ which forms an $l$-co-connected partition on $U^{\prime}=$ $M^{\prime} \backslash F^{\prime}$, the finite intersections of dimension $\geq m-t$ of $\mathcal{P}^{\prime} \mid U^{\prime}$ are $(l-1)$ co-connected and the correspondence between $\mathcal{P}$ and $\mathcal{P}^{\prime}$ defined by sending $P \in \mathcal{P}$ to its modification $P^{\prime} \in \mathcal{P}^{\prime}$ induces a matching of partitions when $\mathcal{P}$ and $\mathcal{P}^{\prime}$ are restricted to $U$ and $U^{\prime}$ respectively.

Moreover, if $\mathcal{W}$ is an open cover of $M$ such that for every $P \in \mathcal{P}$ there is $W \in \mathcal{W}$ such that $\operatorname{st}(P, \mathcal{P}) \subset W$ and the inclusion of $\operatorname{st}(P, \mathcal{P})$ into $W$ induces the zero-homomorphism of the homotopy in dimensions $\leq q-1$, then $\mathcal{P}^{\prime}$ can be constructed so that for each $P \in \mathcal{P}$ and its modification $P^{\prime} \in \mathcal{P}^{\prime}, P^{\prime} \subset \operatorname{st}\left(P, \mathrm{st}^{2} \mathcal{W}\right)$.

A detailed description of the procedure of improving connectivity of intersections is given in Subsections 4.1-4.4.

2.5. Improving the total connectivity of a partition. Let $M$ be a triangulated $(q-1)$-connected $m$-dimensional manifold with $m \geq 2 q+1$ and let $l=m-q+2$. Assume that $F$ is a PL-subcomplex of $M$ lying in Int $M$ and $\mathcal{P}$ is a decomposition of $M$ such that $\operatorname{dim} F \leq m-q$ and $\mathcal{P}$ is an $l$-co-connected partition on $U=M \backslash F$.

Apply 2.4 to improve the connectivity of the elements of $\mathcal{P} \mid U$ to the $(l-1)$-co-connectivity, again apply 2.4 to the modified decomposition to improve the connectivity of the intersections of dimension $m-1$ (of the modified decomposition restricted to the complement of the modified $F$ ) to the $(l-1)$-co-connectivity and thus proceed on the dimension of the intersections $\geq l-1$ until we modify $M$ to an open subset $M^{\prime} \subset M, \mathcal{P}$ to a decomposition $\mathcal{P}^{\prime}$ of $M^{\prime}$ and $F$ to a PL-subcomplex $F^{\prime}$ of $M^{\prime}$ lying in Int $M^{\prime}$ such that $M \backslash M^{\prime}$ is a closed PL-presented subset of $M$, $\operatorname{dim}\left(M \backslash M^{\prime}\right) \leq q$, $\operatorname{dim} F^{\prime} \leq \max \{m-q-1, \operatorname{dim} F\} \leq m-q, \mathcal{P}^{\prime}$ is an $(l-1)$-co-connected partition on $U^{\prime}=M^{\prime} \backslash F^{\prime}$ and $\mathcal{P}$ admits a natural one-to-one correspondence to $\mathcal{P}^{\prime}$ which sends each element of $\mathcal{P}$ to its modification in $\mathcal{P}^{\prime}$ and which becomes a matching of partitions when $\mathcal{P}$ and $\mathcal{P}^{\prime}$ are restricted to $U$ and $U^{\prime}$ respectively. 
2.6. Absorbing simplexes - a summary. Let $M$ be a triangulated $(q-1)$ connected $m$-dimensional manifold with $m \geq 2 q+1$ and let $l=m-q+1$. Assume that $F$ is a PL-subcomplex of $M$ lying in $\operatorname{Int} M$ such that $U=M \backslash F$ is $l$-co-connected $(=(q-1)$-connected $)$ and $\operatorname{dim} F \leq m-q$, and assume that $\mathcal{P}$ is a decomposition of $M$ such that $\mathcal{P}$ is an $l$-co-connected partition on $U$. In Section 4 we will describe a procedure of reducing the dimension of $F$ by 1 by absorbing the $(m-q)$-dimensional simplexes of $F$ into $U$.

Namely, we will show how to modify $M$ to an open subset $M^{\prime}$ of $M$, $F$ to a PL-subcomplex $F^{\prime}$ of $M^{\prime}$ lying in Int $M^{\prime}$ and each $P \in \mathcal{P}$ to a PL-subcomplex $P^{\prime}$ of $M^{\prime}$ so that $F^{\prime}=$ the $(m-q-1)$-skeleton of $F$ with respect to some triangulation of $F, R=M \backslash M^{\prime}$ is a PL-subcomplex of $M$, $\operatorname{dim} R \leq q, \mathcal{P}^{\prime}=\left\{P^{\prime}: P \in \mathcal{P}\right\}$ is a decomposition of $M^{\prime}, \mathcal{P}^{\prime}$ restricted to $U^{\prime}=M^{\prime} \backslash F^{\prime}$ is an $l$-co-connected partition and the natural one-to-one correspondence from $\mathcal{P}$ to $\mathcal{P}^{\prime}$ defined by sending $P \in \mathcal{P}$ to its modification $P^{\prime} \in \mathcal{P}^{\prime}$ becomes a matching of partitions when $\mathcal{P}$ and $\mathcal{P}^{\prime}$ are restricted to $U$ and $U^{\prime}$ respectively.

Moreover, let $\mathcal{W}$ be an open cover of $M$ having the following property: for every $P \in \mathcal{P}$ there are $W \in \mathcal{W}$ and a set $H$ such that $\operatorname{st}(P, \mathcal{P}) \subset H \subset W, H$ is a union of elements of $\mathcal{P}$ and the inclusion $\operatorname{st}(P, \mathcal{P}) \cap U \subset H \cap U$ induces the zero-homomorphism of the homotopy groups in dimensions $\leq q-1$. Then $\mathcal{P}^{\prime}$ can be constructed so that for every $P \in \mathcal{P}$ and its modification $P^{\prime} \in \mathcal{P}^{\prime}, P^{\prime} \subset \operatorname{st}\left(P, \mathrm{st}^{2} \mathcal{W}\right)$.

A detailed description of the procedure of absorbing simplexes is given in Subsection 4.5 (which is based on Subsections 4.1 and 4.3).

2.7. Improving connectivity via a matching. Let $M_{i}$ be $l_{i}$-co-connected triangulated manifolds such that $m_{i}=\operatorname{dim} M_{i} \geq 2\left(m_{i}-l_{i}\right)+3, i=1,2$, and $m_{1}-l_{1}=m_{2}-l_{2}$. Suppose that $\mathcal{P}_{1}$ and $\mathcal{P}_{2}$ are partitions of $M_{1}$ and $M_{2}$ respectively such that there is a matching between $\mathcal{P}_{1}$ and $\mathcal{P}_{2}$, and $\mathcal{P}_{1}$ is $l_{1}$-co-connected. We will show how to modify $M_{2}$ to $M_{2}^{\prime}$ and $\mathcal{P}_{2}$ to $\mathcal{P}_{2}^{\prime}$ so that $M_{2}^{\prime}$ is an open $l_{2}$-co-connected submanifold of $M_{2}, M_{2} \backslash M_{2}^{\prime}$ is a PL-presented closed subset of $M_{2}, \operatorname{dim}\left(M_{2} \backslash M_{2}^{\prime}\right) \leq l_{2}-2$ and $\mathcal{P}_{2}^{\prime}$ is an $l_{2}$-co-connected partition of $M_{2}^{\prime}$ which admits a natural matching to $\mathcal{P}_{2}$ defined by sending each element of $\mathcal{P}_{2}$ to its modification in $\mathcal{P}_{2}^{\prime}$.

By 2.5 modify $M_{2}$ to $M_{2}^{0} \subset M_{2}, \mathcal{P}_{2}$ to $\mathcal{P}_{2}^{0}$ and construct a subset $F^{0}$ of $M_{2}^{0}$ so that $M_{2} \backslash M_{2}^{0}$ is a PL-presented closed subset of $M_{2}$, $\operatorname{dim} M_{2} \backslash M_{2}^{0}$ $\leq 1, F^{0}$ is a PL-subcomplex of $M_{2}^{0}, \operatorname{dim} F^{0} \leq m_{2}-2$, and $\mathcal{P}_{2}^{0}$ is a decomposition of $M_{2}^{0}$ such that $\mathcal{P}_{2}^{0}$ restricted to $M_{2}^{0} \backslash F^{0}$ is an $m_{2}$-co-connected partition that admits a matching to $\mathcal{P}_{2}$ and therefore to $\mathcal{P}_{1}$.

Now assume that for $0 \leq t<m_{2}-l_{2}$ we have constructed $M_{2}^{2 t}, \mathcal{P}_{2}^{2 t}$ and $F^{2 t}$ such that that $M_{2} \backslash M_{2}^{2 t}$ is a PL-presented closed subset of $M_{2}$, $\operatorname{dim}\left(M_{2} \backslash M_{2}^{2 t}\right) \leq t+1, F^{2 t}$ is a PL-subcomplex of $M_{2}^{2 t}, \operatorname{dim} F^{2 t} \leq m_{2}-t-2$, 
and $\mathcal{P}_{2}^{2 t}$ is a decomposition of $M_{2}^{2 t}$ such that $\mathcal{P}_{2}^{2 t}$ restricted to $M_{2}^{2 t} \backslash F^{2 t}$ is an $\left(m_{2}-t\right)$-co-connected partition that admits a matching to $\mathcal{P}_{2}$ and therefore to $\mathcal{P}_{1}$. Proceed to $t+1$ as follows.

By 2.5 modify $M_{2}^{2 t}$ to $M_{2}^{2 t+1} \subset M_{2}^{2 t}, \mathcal{P}_{2}^{2 t}$ to $\mathcal{P}_{2}^{2 t+1}, F^{2 t}$ to $F^{2 t+1}$ so that $M_{2}^{2 t} \backslash M_{2}^{2 t+1}$ is a PL-presented closed subset of $M_{2}^{2 t}, \operatorname{dim}\left(M_{2}^{2 t} \backslash M_{2}^{2 t+1}\right) \leq t+2$, $F^{2 t+1}$ is a PL-subcomplex of $M_{2}^{2 t+1}, \operatorname{dim} F^{2 t+1} \leq m_{2}-t-2, \mathcal{P}_{2}^{2 t+1}$ is a decomposition of $M_{2}^{2 t+1}$, and $\mathcal{P}_{2}^{2 t+1}$ restricted to $M_{2}^{2 t+1} \backslash F^{2 t+1}$ is an $\left(m_{2}-t-1\right)$-co-connected partition that admits a matching to $\mathcal{P}_{2}^{2 t}$ restricted to $M_{2}^{2 t} \backslash F^{2 t}$ and therefore to $\mathcal{P}_{2}$ and $\mathcal{P}_{1}$.

Then since $\mathcal{P}_{1}$ is $l_{1}$-co-connected on the $l_{1}$-co-connected manifold $M_{1}$ we conclude by 2.3 that $M_{2}^{2 t+1} \backslash F^{2 t+1}$ is $\left(m_{2}-t-1\right)$-co-connected. Therefore, by $2.6, M_{2}^{2 t+1}, F^{2 t+1}$ and $\mathcal{P}_{2}^{2 t+1}$ can be modified to $M_{2}^{2 t+2}, F^{2 t+2}$ and $\mathcal{P}_{2}^{2 t+2}$ respectively such that $M_{2}^{2 t+2} \subset M_{2}^{2 t+1}, M_{2}^{2 t+1} \backslash M_{2}^{2 t+2}$ is a PL-subcomplex of $M_{2}^{2 t+1}, \operatorname{dim}\left(M_{2}^{2 t+1} \backslash M_{2}^{2 t+2}\right) \leq t+2, F^{2 t+2}$ is a PL-subcomplex of $M_{2}^{2 t+2}$, $\operatorname{dim} F^{2 t} \leq m_{2}-t-3, \mathcal{P}_{2}^{2 t+2}$ is a decomposition of $M_{2}^{2 t+2}$, and $\mathcal{P}_{2}^{2 t+2}$ restricted to $M_{2}^{2 t+2} \backslash F^{2 t+2}$ is an $\left(m_{2}-t-1\right)$-co-connected partition that admits a matching to $\mathcal{P}_{2}^{2 t+1}$ restricted to $M_{2}^{2 t+1} \backslash F^{2 t+1}$ and therefore to $\mathcal{P}_{2}$ and $\mathcal{P}_{1}$.

Then for $t=m_{2}-l_{2}$ we have $M_{2}^{\prime}=M_{2}^{2 t} \backslash F^{2 t}$, and $\mathcal{P}_{2}^{\prime}=\mathcal{P}_{2}^{2 t}$ restricted to $M_{2}^{\prime}$ will have the required properties (note that $m_{2}-l_{2}+1 \leq l_{2}-2$ and therefore $\left.\operatorname{dim}\left(M_{2} \backslash M_{2}^{\prime}\right) \leq l_{2}-2\right)$.

2.8. Moving to a rational position. Let $M$ be a triangulated manifold with the rational structure determined by a triangulation $\mathcal{T}$ (see Section 1). Assume that $\mathcal{T}^{\prime}$ is a (not necessarily rational) triangulation of $M$. We will show that the identity map of $M$ can be arbitrarily closely approximated by a PL-homeomorphism $f: M \rightarrow M$ such that for very $\Delta^{\prime} \in \mathcal{T}^{\prime}, f\left(\Delta^{\prime}\right)$ is a rational subcomplex of $M$.

Embed $M$ into a Hilbert space by a map which is linear on every simplex of $\mathcal{T}$ and refer to this Hilbert space when properties of linearity are used. Let $\mathcal{T}^{\prime \prime}$ be a triangulation of $M$ such that $\mathcal{T}^{\prime \prime}$ is a subdivision of both $\mathcal{T}$ and $\mathcal{T}^{\prime}$, and the simplexes of $\mathcal{T}^{\prime \prime}$ are linear. Approximate every vertex $v$ of $\mathcal{T}^{\prime \prime}$ by a rational point $p_{v}(=$ a point with rational barycentric coordinates with respect to $\mathcal{T}$ ) such that for every simplex $\Delta$ of $\mathcal{T}, p_{v} \in \Delta$ if and only if $v \in \Delta$. The approximation of the vertices of $\mathcal{T}^{\prime \prime}$ can be extended to the PL-map $f: M \rightarrow M$ sending each vertex $v$ of $\mathcal{T}^{\prime \prime}$ to $p_{v}$ so that $f$ is linear on each simplex of $\mathcal{T}^{\prime \prime}$. If $p_{v}$ is sufficiently close to $v$ for every vertex $v \in \mathcal{T}^{\prime \prime}$ then the map $f$ is a PL-homeomorphism that can be chosen to be arbitrarily close to the identity map. Clearly, $f$ sends every simplex of $\mathcal{T}^{\prime \prime}$ to a rational simplex and therefore $f$ sends every simplex of $\mathcal{T}^{\prime}$ to a rational subcomplex of $M$.

Let $M$ be an open subset of a space $Y$ and $d$ a metric on $Y$. We can assume that the homeomorphism $f: M \rightarrow M$ constructed above satisfies 
$d(y, f(y)) \leq d(y, Y \backslash M)$ for every $y \in M$. Then $f$ extends to the homeomorphism $g: Y \rightarrow Y$ such that $g(y)=y$ if $y \in Y \backslash M$ and $g(y)=f(y)$ if $y \in M$. Now assume that $R$ is a PL-presented subset of $M$ and let $R_{1} \subset R_{2} \subset \cdots \subset R_{n}$ be closed subsets of $M$ such that $R=R_{n}, R_{1}$ is a PL-subcomplex of $M$ and $R_{i+1} \backslash R_{i}$ is a PL-subcomplex of $M \backslash R_{i}$, $i=1, \ldots, n-1$. Let us show that the identity map of $Y$ can be arbitrarily closely approximated by a homeomorphism $g: Y \rightarrow Y$ such that $g(y)=y$ for every $y \in Y \backslash M, g(R)$ is a closed rationally presented subset of $M$, and $g$ restricted to $M \backslash R$ is a PL-homeomorphism to $M \backslash g(R)$ sending $\mathcal{P}$ to a rational decomposition of $M \backslash g(R)$ (note that the rational structure of $M$ induces the corresponding rational structure on open subsets of $M$ such that the inclusions are rational maps).

Approximate the identity map of $Y$ by a homeomorphism $g_{1}: Y \rightarrow Y$ such that $g_{1}$ does not move the points of $Y \backslash M, g_{1}$ restricted to $M$ is a PL-homeomorphism and $g_{1}\left(R_{1}\right)$ is a rational subcomplex of $M$. Approximate $g_{1}$ by a map $g_{2}: Y \rightarrow Y$ such that $g_{2}$ does not move the points of $(Y \backslash M) \cup g_{1}\left(R_{1}\right), g_{2}$ restricted to $M \backslash g_{1}\left(R_{1}\right)$ is a PL-homeomorphism and $g_{2}\left(g_{1}\left(R_{2} \backslash R_{1}\right)\right)$ is a rational subcomplex of $M \backslash g_{1}\left(R_{1}\right)$. Proceed by induction and construct for every $i=1, \ldots, n-1$ an approximation of the identity map of $Y$ by a homeomorphism $g_{i+1}: Y \rightarrow Y$ such that $g_{i+1}$ does not move the points of $(Y \backslash M) \cup\left(g_{i} \circ \cdots \circ g_{1}\right)\left(R_{i}\right), g_{i+1}$ restricted to $M \backslash\left(g_{i} \circ \cdots \circ g_{1}\right)\left(R_{i}\right)$ is a PL-homeomorphism and $\left(g_{i+1} \circ \cdots \circ g_{1}\right)\left(R_{i+1} \backslash R_{i}\right)$ is a rational subcomplex of $M \backslash\left(g_{i} \circ \cdots \circ g_{1}\right)\left(R_{i}\right)$. Finally, construct $g_{n}$ with the additional property that $g=g_{n} \circ \cdots \circ g_{1}$ sends $\mathcal{P}$ to a rational decomposition of $M \backslash g(R)$. Then $g$ will have the required properties.

3. Proof of the $Z$-set unknotting theorem. A map $\psi: X \rightarrow \Gamma$ between two spaces $X$ and $\Gamma$ is said to be $U V^{n-1}$ if $\psi(X)$ is dense in $\Gamma$ and, for every point $p \in \Gamma$ and every neighborhood $U$ of $p$, there is a smaller neighborhood $V$ of $p$ such that the inclusion of $\psi^{-1}(V)$ into $\psi^{-1}(U)$ induces the zero-homomorphism of the homotopy groups in dimensions $\leq n-1$. In this section we will prove the following $Z$-set unknotting theorem which is slightly stronger than Theorem 1.2.

Theorem 3.1. Let $X_{1}$ and $X_{2}$ be $n$-dimensional Nöbeling spaces and let $A_{1}$ and $A_{2}$ be homeomorphic $Z$-sets in $X_{1}$ and $X_{2}$ respectively such that $X_{1} \backslash A_{1}$ and $X_{2} \backslash A_{2}$ are homeomorphic to Nöbeling spaces modeled on triangulated manifolds. Then every homeomorphism $f_{A}: A_{1} \rightarrow A_{2}$ extends to a homeomorphism $f_{X}: X_{1} \rightarrow X_{2}$.

Moreover, for any $U V^{n-1}$-maps $\psi_{1}: X_{1} \rightarrow \Gamma$ and $\psi_{2}: X_{2} \rightarrow \Gamma$ to a space $\Gamma$ and every open cover $\mathcal{O}^{+}$of $\Gamma$ there is an open cover $\mathcal{O}^{-}$of $\Gamma$ such that $\mathcal{O}^{-}$does not depend on $A_{1}, A_{2}$ and $f_{A}$ and $\mathcal{O}^{-}$has the following 
property: if $\psi_{1}$ restricted to $A_{1}$ and $\psi_{2} \circ f_{A}$ are $\mathcal{O}^{-}$-close then $f_{X}$ can be constructed so that $\psi_{1}$ and $\psi_{2} \circ f_{X}$ are $\mathcal{O}^{+}$-close.

The proof of Theorem 3.1 is based on a few propositions; before each proposition or its proof we present notions, notations and properties used in that proposition.

Let $M$ be a triangulated space and let $P$ be either a rational subcomplex or an open subset of $M$. We always consider $P$ with the induced rational structure for which the inclusion of $P$ into $M$ is a rational map. Then there are rational triangulations of $P$ and $M$ respectively such that the simplexes of $P$ are linearly and rationally embedded in the simplexes of $M$. Now we can derive from the interpretation of $M(k)$ given in Section 1 that $P \cap M(k)=P(k)$.

Let $M$ be a triangulated manifold. It can also be derived from the interpretation of $M(k)$ given in Section 1 that $M \backslash M(k)$ is a countable union of simplexes of dimension $\leq k$ which are PL-embedded in $M$. Then for an integer $q$ such that $\operatorname{dim} M \geq q+k$ every map $f$ from a $(q-1)$-dimensional sphere $S^{q-1}$ to $M$ can be arbitrarily closely approximated by a map into $M(k)$ and therefore $f$ can be homotoped into $M(k)$. In particular, this implies that if $M(k)$ is $(q-1)$-connected and $M^{\prime}$ is an open subset of $M$ such that $M(k) \subset M^{\prime}$ then, since $M^{\prime}(k)=M(k)$, we deduce that $M^{\prime}$ is $(q-1)$-connected as well.

Let $A$ be a closed subset of a space $X$. A collection $\mathcal{C}$ of subsets $X \backslash A$ is said to properly approach $A$ if for every sequence $\left\{C_{j}\right\}$ of elements of $\mathcal{C}$ such that there is a sequence of points $x_{j} \in C_{j}$ converging to a point $a \in A$ we have $\lim _{j \rightarrow \infty} C_{j}=a$ (that is, $\lim _{j \rightarrow \infty} y_{j}=a$ for every sequence $\left\{y_{j}\right\}$ with $y_{j} \in C_{j}$ ). Note that if $\mathcal{C}$ properly approaches $A$ then the closure in $X$ of no $C \in \mathcal{C}$ intersects $A$. Also if collections $\mathcal{C}$ and $\mathcal{C}^{\prime}$ of subsets of $X \backslash A$ properly approach $A$ then $\operatorname{st}\left(\mathcal{C}, \mathcal{C}^{\prime}\right)$ properly approaches $A$ as well.

Let $X$ be a subspace of a space $Y$. Saying that $\mathcal{U}$ is an open cover of $X$ we mean that $\mathcal{U}$ is a cover of $X$ by subsets open in $X$.

Let $X$ be a dense subset of a space $Y$ and $U$ an open subset of $X$. By the extension $U_{Y}$ of $U$ to $Y$ we understand the largest open subset $U_{Y}$ of $Y$ such that $U=U_{Y} \cap X$. By the extension to $Y$ of a collection $\mathcal{U}$ of open subsets of $X$ we mean the collection of the extensions to $Y$ of the elements of $\mathcal{U}$. Note that if $A$ is a closed subset of $X$ such that $A$ is also closed in $Y$, and $\mathcal{U}$ is a collection of open subsets of $X \backslash A$ such that $\mathcal{U}$ properly approaches $A$, then for the extension $\mathcal{U}_{Y}$ of $\mathcal{U}$ to $Y$ we deduce that $\mathcal{U}_{Y}$ properly approaches $A$ as well.

Let $A_{1}$ and $A_{2}$ be homeomorphic subsets of spaces $Y_{1}$ and $Y_{2}$ respectively, let $f_{A}: A_{1} \rightarrow A_{2}$ be a homeomorphism and let $\mu: \mathcal{C}_{1} \rightarrow \mathcal{C}_{2}$ be a one-toone correspondence between collections of subsets of $Y_{1} \backslash A_{1}$ and $Y_{2} \backslash A_{2}$ 
respectively. We say that $\mu$ agrees with $f_{A}: A_{1} \rightarrow A_{2}$ if for every sequence $C_{j}, j=1,2, \ldots$, of elements of $\mathcal{C}_{1}, C_{j}$ converge to $a \in A_{1}$ in $Y_{1}($ as $j \rightarrow \infty)$ if and only $\mu\left(C_{j}\right)$ converge to $f_{A}(a)$ in $Y_{2}$. It is easy to check that if $\mathcal{C}_{1}$ and $\mathcal{C}_{2}$ properly approach $A_{1}$ and $A_{2}$ respectively, $\mu$ agrees with $f_{A}$ and a collection $\mathcal{C}$ of subsets of $Y_{1} \backslash A_{1}$ properly approaches $A_{1}$, then $\mu\left(\operatorname{st}\left(\mathcal{C}, \mathcal{C}_{1}\right)\right)$ properly approaches $A_{2}$, where $\mu\left(\operatorname{st}\left(\mathcal{C}, \mathcal{C}_{1}\right)\right)=\left\{\mu\left(\operatorname{st}\left(C, \mathcal{C}_{1}\right)\right): C \in \mathcal{C}\right\}$ and $\mu\left(\operatorname{st}\left(C, \mathcal{C}_{1}\right)\right)=\bigcup\left\{\mu\left(C^{\prime}\right): C^{\prime} \in \mathcal{C}_{1}, C^{\prime} \cap C \neq \emptyset\right\}$.

Let $X_{i}, i=1,2$, be subsets $X_{i} \subset Y_{i}$ such that $X_{i}$ is dense in $Y_{i}$ and let $\psi_{i}: X_{i} \rightarrow \Gamma$ be maps to a space $\Gamma$ and $\mathcal{O}$ an open cover of $\Gamma$. We say that $\mu, \psi_{1}$ and $\psi_{2}$ agree with respect to $\mathcal{O}$ if for every $C \in \mathcal{C}_{1}$ there is an element $\Omega \in \mathcal{O}$ such that $C$ is contained in the extension of $\psi_{1}^{-1}(\Omega)$ to $Y_{1}$ and $\mu(C)$ is contained in the extension of $\psi_{2}^{-1}(\Omega)$ to $Y_{2}$

Let $M$ be a triangulated manifold. Then $(\partial M)(k)$ is a $Z$-set in $M(k)$. Indeed, take an open cover $\mathcal{U}$ of $M(k)$ and let $\mathcal{U}_{M}$ be the extension of $\mathcal{U}$ to $M$ and $M^{\prime}$ the union of the elements of $\mathcal{U}_{M}$. The identity map of $M^{\prime}$ can be arbitrarily closely approximated by a PL-embedding $f$ of $M^{\prime}$ into Int $M^{\prime}$ such that $f\left(M^{\prime}\right)$ is a PL-subcomplex of $M^{\prime}$. Then, by 2.8 , we may assume that $f$ is rational and $\mathcal{U}_{M}$-close to the identity map of $M^{\prime}$, and hence $f$ induces a $\mathcal{U}$-close map from $M(k)$ into $(\operatorname{Int} M)(k)$.

Suppose that $A$ is a closed subset of $X$ and $X \backslash A$ is embedded in a space $M$. Let us show that there are an open subset $V$ of $M$ containing $X \backslash A$ and a space $Y$ such that $X$ and $V$ embed into $Y$ so that $Y=X \cup V$, $A=Y \backslash V$ and $A$ is closed in $Y$.

Let $H$ be a Hilbert space. Consider the space $H \times[0,1]$. Let $\mathcal{U}$ be an open cover of $H \times(0,1]$ such that $\mathcal{U}$ properly approaches $H \times\{1\}$. Take any embedding $e: X \rightarrow H \times[0,1]$ such that $e(A) \subset H \times\{1\}$ and $e(X \backslash A) \subset$ $H \times(0,1]$. By Walsh's lemma $e \mid X \backslash A$ can be $\mathcal{U}$-closely approximated by a map $g_{X \backslash A}: X \backslash A \rightarrow H \times(0,1]$ such that $g_{X \backslash A}$ extends over an open subset $X \backslash A \subset V$ of $M$ to a map $g_{V}: V \rightarrow H \times(0,1]$. Approximate $g_{V}$ by a $\mathcal{U}$-close embedding $h_{V}: V \rightarrow H \times(0,1]$ and define $h: Y=A \cup V \rightarrow H \times[0,1]$ by $h(y)=e(y)$ if $y \in A$ and $h(y)=h_{V}(y)$ if $y \in V$. Now we can transfer the topology of $h(Y)$ to $Y$ and it is easy to see that then $Y$ has the required properties.

Assume that $X_{i}$ and $A_{i}, i=1,2$, satisfy the assumptions of Theorem 3.1, let $f_{A}: A_{1} \rightarrow A_{2}$ be a homeomorphism and let $X_{i} \backslash A_{i}$ be homeomorphic to $M_{i}\left(k_{i}\right)$, where $M_{i}$ is an $l_{i}$-co-connected triangulated manifold such that $n=m_{i}-l_{i}+1, k_{i}=l_{i}-2$ and $m_{i} \geq 2\left(m_{i}-l_{i}\right)+3$ for $n=\operatorname{dim} X_{1}=\operatorname{dim} X_{2}$ and $m_{i}=\operatorname{dim} M_{i}$ (note that by Theorem 1.1, $M_{i}\left(k_{i}\right)$ is an $n$-dimensional Nöbeling space). Identify $X_{i} \backslash A_{i}$ with $M_{i}\left(k_{i}\right)$ and, by the property proved above, replace $M_{i}$ by an open subset of $M_{i}$ containing $M_{i}\left(k_{i}\right)$ and assume that $X_{i}$ and $M_{i}$ are subspaces of a space $Y_{i}$ such that $Y_{i}=X_{i} \cup M_{i}, A_{i}=$ $Y_{i} \backslash M_{i}$ and $A_{i}$ is closed in $Y_{i}$. 
Proposition 3.2. Let $X_{i}, A_{i}, f_{A}, Y_{i}, M_{i}, k_{i}, l_{i}, m_{i}, n$ be as above. Then for every $l_{1}$-co-connected rational partition $\mathcal{P}_{1}$ of an open subset $M_{1}^{\prime}$ of $M_{1}$ such that $M_{1}\left(k_{1}\right) \subset M_{1}^{\prime}, \mathcal{P}_{1}$ properly approaches $A_{1}$ and $\mathcal{P}_{1}$ contains no finite intersections of dimension $\leq l_{1}-2$, there are an open subset $M_{2}^{\prime}$ of $M_{2}$ and an $l_{2}$-co-connected rational partition $\mathcal{P}_{2}$ of $M_{2}^{\prime}$ such that $M_{2}\left(k_{2}\right) \subset M_{2}^{\prime}$, $\mathcal{P}_{2}$ properly approaches $A_{2}, \mathcal{P}_{2}$ contains no finite intersections of dimension $\leq l_{2}-2$ and $\mathcal{P}_{1}$ and $\mathcal{P}_{2}$ admit a matching $\mu: \mathcal{P}_{1} \rightarrow \mathcal{P}_{2}$ which agrees with $f_{A}$.

Moreover, for any $U V^{n-1}$-maps $\psi_{1}: X_{1} \rightarrow \Gamma$ and $\psi_{2}: X_{2} \rightarrow \Gamma$ to a space $\Gamma$ and every open cover $\mathcal{O}^{+}$of $\Gamma$ there is an open cover $\mathcal{O}^{-}$of $\Gamma$ which refines $\mathcal{O}^{+}$such that $\mathcal{O}^{-}$does not depend on $A_{1}, A_{2}$ and $f_{A}$ and $\mathcal{O}^{-}$ has the following property: if $\psi_{1} \mid A_{1}$ and $\psi_{2} \circ f_{A}$ are $\mathcal{O}^{-}$-close and $\mathcal{P}_{1}$ refines the extension of $\psi_{1}^{-1}\left(\mathcal{O}^{-}\right)$to $Y_{1}$ then $M_{2}^{\prime}, \mathcal{P}_{2}$ and $\mu$ can be constructed so that $\mu, \psi_{1}$ and $\psi_{2}$ agree with respect to $\mathcal{O}^{+}$.

Let us show how Theorem 3.1 can be derived from Proposition 3.2.

Proof of Theorem 3.1. Assume that the conclusion holds in dimensions $\leq n-1$ and let us prove it in dimensions $n$. Fix complete metrics on $X_{1}$ and $X_{2}$ and let $\varepsilon>0$. Take an open cover $\mathcal{U}$ of $X_{1} \backslash A_{1}$ of mesh $\mathcal{U}<\varepsilon$ such that $\mathcal{U}$ properly approaches $A_{1}$. Denote by $\mathcal{U}_{M}$ the extension to $M_{1}$ of $\mathcal{U}$ and let $M_{1}^{\prime}$ be the union of the elements of $\mathcal{U}_{M}$. Take a rational triangulation of $M_{1}^{\prime}$ such that for the partition $\mathcal{P}_{1}$ of $M_{1}^{\prime}$ formed by the stars of the vertices with respect to the first barycentric subdivision of the triangulation, $\mathcal{P}_{1}$ refines $\mathcal{U}_{M}$. Then $\mathcal{P}_{1}$ is a rational partition, $\mathcal{P}_{1}$ properly approaches $A_{1}$ and for every $P \in \mathcal{P}_{1}$, diam $P \cap X_{1}<\varepsilon$. Since the intersections of $\mathcal{P}_{1}$ of dimension $\leq l_{1}-2$ do not intersect $M_{1}\left(k_{1}\right)$ we can remove them from $M_{1}^{\prime}$ and the elements of $\mathcal{P}_{1}$, and assume that $\mathcal{P}_{1}$ has no non-empty intersections of dimension $\leq l_{1}-2$. Note that all the intersections of $\mathcal{P}_{1}$ remain contractible and hence $\mathcal{P}_{1}$ is $l_{1}$-co-connected.

By Proposition 3.2 there are an open submanifold $M_{2}^{\prime}$ of $M_{2}$ and an $l_{2^{-}}$ co-connected rational partition of $M_{2}^{\prime}$ such that $M_{2}\left(k_{2}\right) \subset M_{2}^{\prime}, \mathcal{P}_{2}$ properly approaches $A_{2}$ and $\mathcal{P}_{2}$ admits a matching $\mu: \mathcal{P}_{1} \rightarrow \mathcal{P}_{2}$ which agrees with $f_{A}: A_{1} \rightarrow A_{2}$.

We are going to construct homeomorphisms $f_{P}: P\left(k_{1}\right) \rightarrow \mu(P)\left(k_{2}\right)$ for all finite intersections $P$ of $\mathcal{P}_{1}$ of dimension $\leq m_{1}-1$ which will agree on the common intersections. Let $t \leq m_{1}-1$ and assume that for every finite intersection $P$ of dimension $\leq t-1$ we already constructed $f_{P}$. Take an intersection $P$ of $\mathcal{P}_{1}$ such that $\operatorname{dim} P=t$. By Theorem 1.1, $P\left(k_{1}\right)$ is a Nöbeling space of dimension $\leq n-1$. Note that for the union $P^{\prime}$ of the intersections of $\mathcal{P}_{1}$ of dimension $<t$ that are contained in $P, P^{\prime}$ lies in $\partial P$ and hence $P^{\prime}\left(k_{1}\right)$ is a $Z$-set in $P\left(k_{1}\right)$. Define the homeomorphism $f_{P^{\prime}}$ : $P^{\prime}\left(k_{1}\right) \rightarrow \mu\left(P^{\prime}\right)\left(k_{2}\right)$ by the homeomorphisms of the intersections forming $P^{\prime}$. According to our assumption Theorem 3.1 holds in dimensions $\leq n-1$. Therefore $f_{P^{\prime}}$ can be extended to a homeomorphism $f_{P}: P\left(k_{1}\right) \rightarrow \mu(P)\left(k_{2}\right)$. 
Recall that $\mathcal{P}_{1}$ and $\mathcal{P}_{2}$ properly approach $A_{1}$ and $A_{2}$ respectively and the matching $\mu$ agrees with $f_{A}$. Then a homeomorphism extending $f_{X}$ between $X_{1}$ and $X_{2}$ can be obtain by pasting homeomorphisms from $P\left(k_{1}\right)$ to $\mu(P)\left(k_{2}\right)$ for $P \in \mathcal{P}_{1}$ which extend the already defined homeomorphisms on intersections of $\mathcal{P}_{1}$ of dimension $\leq m_{1}-1$.

Fix $P \in \mathcal{P}_{1}$ and let $P^{\prime}$ be the union of the intersections of $\mathcal{P}_{1}$ of dimension $\leq m_{1}-1$ that are contained in $P$. The homeomorphism $f_{P^{\prime}}: P^{\prime}\left(k_{1}\right) \rightarrow$ $\mu\left(P^{\prime}\right)\left(k_{2}\right)$ is a homeomorphism of $Z$-subsets of $P\left(k_{1}\right)$ and $\mu(P)\left(k_{2}\right)$ respectively. Therefore we can repeat for $P$ and $\mu(P)$ the same procedure that we did for $M_{1}$ and $M_{2}$ but this time in the opposite direction from $\mu(P)$ to $P$, first "splitting" $\mu(P)$ into small pieces and then defining the corresponding splitting of $P$.

Thus going back and forth we are able after each iteration to extend $f_{A}$ to a partial homeomorphism of larger and larger parts of $X_{1}$ and $X_{2}$ and simultaneously to restrict for each point of $X_{1}$ and $X_{2}$ the set to which this point can be sent under a possible extension of $f$. Finally, passing to the limit and using the completeness of $X_{1}$ and $X_{2}$ we get the desired homeomorphism $f_{X}: X_{1} \rightarrow X_{2}$. It is clear from the construction that $f_{X}\left(P \cap X_{1}\right)=\mu(P) \cap X_{2}$ for every $P \in \mathcal{P}_{1}$.

Now let $\psi_{i}: X_{i} \rightarrow \Gamma, i=1,2$, be $U V^{n-1}$-maps to a space $\Gamma$ and $\mathcal{O}^{+}$an open cover of $\Gamma$. Assume that an open cover $\mathcal{O}^{-}$of $\Gamma$ satisfies the conclusions of Proposition 3.2 and assume that $\psi_{1} \mid A$ and $\psi_{2} \circ f_{A}$ are $\mathcal{O}^{-}$-close. Then we can take the cover $\mathcal{U}$ of $X_{1} \backslash A_{1}$ such that $\mathcal{U}$ refines $\psi_{1}^{-1}\left(\mathcal{O}^{-}\right)$and will find that $\mathcal{P}_{1}$ refines the extension of $\psi_{1}^{-1}\left(\mathcal{O}^{-}\right)$to $Y_{1}$. Now, by Proposition 3.2, we can construct $M_{2}^{\prime}, \mathcal{P}_{2}$ and $\mu$ so that $\mu, \psi_{1}$ and $\psi_{2}$ agree with respect to $\mathcal{O}^{+}$. Then for every $P \in \mathcal{P}_{1}$ there is an element of $\mathcal{O}^{+}$containing both $\psi_{1}\left(P \cap X_{1}\right)$ and $\psi_{2}\left(\mu(P) \cap X_{2}\right)$ and, since $f_{X}\left(P \cap X_{1}\right)=\mu(P) \cap X_{2}$ for every $P \in \mathcal{P}_{1}$, we see that the maps $\psi_{1}$ and $\psi_{2} \circ f_{X}$ are $\mathcal{O}^{+}$-close.

In the proof of Proposition 3.2 we will use the following propositions and facts.

A map $\phi: Y \rightarrow X$ is said to be a $Z$-embedding if $\phi$ is a closed embedding of $Y$ into $X$ and $\phi(Y)$ is a $Z$-set in $X$. Let $X$ be a Nöbeling space. It is well-known that if $Y$ is a complete space of dimension $\leq n, A$ is a closed subset of $Y$ and $f: A \rightarrow X$ is a $Z$-embedding, then every extension of $f$ over $Y$ can be arbitrarily closely approximated by an extension which is a $Z$-embedding. It is also well-known (and can be derived from the previous fact) that for every $Z$-set $A \subset X$ the identity map on $X$ can be arbitrarily closely approximated by a $Z$-embedding whose image misses $A$. Another simple property of $Z$-sets says that if a closed subset $A$ of $X$ is a countable union of $Z$-sets then $A$ is a $Z$-set as well. 
We say that for two collections $\mathcal{V}$ and $\mathcal{U}$ of subsets of a space $X, \mathcal{V}$ is an $(n-1)$-refinement of $\mathcal{U}$, written $\mathcal{V} \prec_{n-1} \mathcal{U}$, if for every $V \in \mathcal{V}$ there is $U \in \mathcal{U}$ such that $V \subset U$ and the inclusion of $V$ into $U$ induces the zero-homomorphism of the homotopy groups in dimensions $\leq n-1$.

Proposition 3.3. Let $X$ be an $n$-dimensional Nöbeling space and let $\psi: X \rightarrow \Gamma$ be a $U V^{n-1}$-map. Then for every open cover $\mathcal{U}^{+}$of $\Gamma$ there is an open cover $\mathcal{U}^{-}$of $\Gamma$ having the following property: for every complete space $Y$ of dimension $\leq n$, a closed subset $A$ of $Y$, a map $f: Y \rightarrow \Gamma$ and a $Z$-embedding $\phi_{A}: A \rightarrow X$ such that $f$ restricted to $A$ and $\psi \circ \phi_{A}$ are $\mathcal{U}^{-}$-close, we can extend $\phi_{A}$ to a $Z$-embedding $\phi: Y \rightarrow X$ such that $f$ and $\psi \circ \phi$ are $\mathcal{U}^{+}$-close.

Proof. Let open covers $\mathcal{U}^{0}, \mathcal{U}^{1}, \ldots, \mathcal{U}^{n+2}$ of $\Gamma$ be such that st $\mathcal{U}^{i}$ refines $\mathcal{U}^{i+1}$, st $\psi^{-1}\left(\mathcal{U}^{i}\right) \prec_{n-1} \psi^{-1}\left(\mathcal{U}^{i+1}\right), 0 \leq i<n+2$, and $\mathcal{U}^{n+2}=\mathcal{U}^{+}$. Note that since $\psi(X)$ is dense in $\Gamma$ we have st $\psi^{-1}\left(\mathcal{U}^{i}\right)=\psi^{-1}\left(\operatorname{st} \mathcal{U}^{i}\right)$. Set $\mathcal{U}^{-}=\mathcal{U}^{0}$ and assume that $f \mid A$ and $\psi \circ \phi_{A}$ are $\mathcal{U}^{0}$-close. Since $\operatorname{dim} Y \leq n$ there is a surjective map $p: Y \rightarrow Y^{\prime}$ such that $p^{-1}(p(A))=A, p \mid A$ is a homeomorphism between $A$ and $p(A), K=Y^{\prime} \backslash p(A)$ is a simplicial complex of dimension $\leq n$, the collection $\mathcal{T}$ of the simplexes of $K$ properly approaches $A$ and $p^{-1}(\mathcal{T})$ refines $f^{-1}\left(\mathcal{U}^{0}\right)$. Identify $A$ with $p(A)$ and consider $A$ as a subset of $Y^{\prime}$.

We will construct a continuous extension $\phi^{\prime}$ of $\phi_{A}$ over $Y^{\prime}$. Fix a metric $d$ on $Y^{\prime}$. For every 0-dimensional simplex (= vertex) $\Delta$ of $K$ choose a point $a_{\Delta} \in A$ such that $d\left(a_{\Delta}, \Delta\right) \leq 2 d(A, \Delta)$. If there is $U \in \mathcal{U}^{0}$ such that $p^{-1}(\Delta) \subset f^{-1}(U)$ and $\phi_{A}\left(a_{\Delta}\right) \in \psi^{-1}(U)$ then define $\phi^{\prime}(\Delta)=\phi_{A}\left(a_{\Delta}\right)$, otherwise choose $U \in \mathcal{U}^{0}$ such that $p^{-1}(\Delta) \subset f^{-1}(U)$ and define $\phi^{\prime}(\Delta)$ as any point in $\psi^{-1}(U)$. It is easy to see that, since $f \mid A$ and $\psi \circ \phi_{A}$ are $\mathcal{U}^{0}$-close, $\phi^{\prime}$ is continuous on $A \cup K^{0}$, where $K^{i}$ is the $i$-skeleton of $K$ (= the union of the simplexes of dimension $\leq i$ ). Assume that $\phi^{\prime}$ is already defined on $A \cup K^{i}, i \leq n-1$, and such that the images of the simplexes of $K$ of dimension $\leq i$ refine $\psi^{-1}\left(\mathcal{U}^{i}\right)$.

Let $\Delta$ be an $(i+1)$-dimensional simplex of $K$. Fix a metric on $X$ and define $d_{\Delta}=\inf \left\{\operatorname{diam} C: \phi^{\prime}(\partial \Delta) \subset C, \phi^{\prime} \mid \partial \Delta\right.$ is null-homotopic in $C$ and there is $U \in \mathcal{U}^{i+1}$ such that $\left.C \subset \psi^{-1}(U)\right\}$. Since st $\psi^{-1}\left(\mathcal{U}^{i}\right) \prec_{n-1}$ $\psi^{-1}\left(\mathcal{U}^{i+1}\right)$ we conclude that $d_{\Delta}$ is well-defined. Extend $\phi^{\prime}$ from $\partial \Delta$ to $\Delta$ so that $\operatorname{diam} \phi^{\prime}(\Delta) \leq d_{\Delta}+d(A, \Delta)$ and $\phi^{\prime}(\Delta)$ is contained in an element of $\psi^{-1}\left(\mathcal{U}^{i+1}\right)$. Thus we extend $\phi^{\prime}$ over $A \cup K^{i+1}$ and this extension is continuous. Indeed, if a sequence $\Delta_{j}$ of $(i+1)$-dimensional simplexes of $K$ converges to a point $a \in A$ then $\phi^{\prime}\left(\partial \Delta_{j}\right)$ converges to $\phi^{\prime}(a)$ and hence $d_{\Delta_{j}}$ converges to 0 because $X$ is locally $(n-1)$-connected. Then $\phi^{\prime}\left(\Delta_{j}\right)$ converges to $\phi^{\prime}(a)$ and hence $\phi^{\prime}$ is continuous on $A \cup K^{i+1}$. Clearly, the images of the $(i+1)$-dimensional simplexes of $K$ refine $\psi^{-1}\left(\mathcal{U}^{i+1}\right)$. Thus, by induction, we can extend $\phi_{A}$ to $\phi^{\prime}: Y^{\prime}=A \cup K \rightarrow X$ such that $\phi^{\prime}(\mathcal{T})$ refines $\psi^{-1}\left(\mathcal{U}^{n}\right)$. 
Since $f\left(p^{-1}(\Delta)\right)$ and $\psi\left(\phi^{\prime}(\Delta)\right)$ are $\mathcal{U}^{0}$-close for every 0-dimensional simplex $\Delta$ of $K$, and since $p^{-1}(\mathcal{T})$ refines $f^{-1}\left(\mathcal{U}^{0}\right)$ and $\phi^{\prime}(\mathcal{T})$ refines $\psi^{-1}\left(\mathcal{U}^{n}\right)$, we deduce that $f$ and $\psi \circ \phi^{\prime} \circ p$ are $\operatorname{st}\left(\mathcal{U}^{0}, \mathcal{U}^{n}\right)$-close, and hence $f$ and $\psi \circ \phi^{\prime} \circ p$ are $\mathcal{U}^{n+1}$-close. Now we can approximate $\phi^{\prime} \circ p$ by a $Z$-embedding $\phi: Y \rightarrow X$ which coincides with $\phi_{A}$ on $A$ such that $f$ and $\psi \circ \phi$ are $\mathcal{U}^{n+2}$-close $\left(=\mathcal{U}^{+}\right.$-close $)$and we are done.

Let $X$ be an $n$-dimensional Nöbeling space and $\mathcal{U}$ an open cover of $X$. It is well-known (and can be easily shown) that if two maps from a space $Y$ of dimension $\leq n-1$ into $X$ are sufficiently close then they are $\mathcal{U}$-homotopic (= there is a homotopy $H: Y \times[0,1] \rightarrow X$ which connects the maps such that for every $y \in Y, H(y \times[0,1])$ is contained in an element of $\mathcal{U})$.

Proposition 3.4. Let $X$ be an n-dimensional Nöbeling space, let $A$ be a $Z$-set in $X$ and let $\mathcal{C}$ be a cover of $X \backslash A$ that properly approaches $A$. Then there is an open cover $\mathcal{V}$ of $X \backslash A$ such that $\mathcal{V}$ properly approaches $A$ and $\mathcal{C} \prec_{n-1} \mathcal{V}$. Moreover, if $\mathcal{W}$ is an open cover of $X$ such that $\mathcal{C} \prec_{n-1} \mathcal{W}$ then $\mathcal{V}$ can be constructed so that $\mathcal{V}$ refines $\mathrm{st}^{3} \mathcal{W}$.

Proof. Fix a metric $d$ on $X$ such that $d(x, y) \leq 1$ for every $x, y \in X$. If $C \in \mathcal{C}$ is not a singleton, write $d_{C}$ for the infimum of $\operatorname{diam}(G)$ for open subsets $G$ of $X$ such that $C \subset G, G$ is contained in an element of $\mathcal{W}$ and the inclusion $C \subset G$ induces the zero-homomorphism of the homotopy groups in dimensions $\leq n-1$. If $C \in \mathcal{C}$ is a singleton, define $d_{C}=d(C, A)$. For every $C \in \mathcal{C}$ fix an open set $G_{C}$ such that $\operatorname{diam}\left(G_{C}\right) \leq 2 d_{C}, G$ is contained in an element of $\mathcal{W}$ and the inclusion $C \subset G_{C}$ induces the zero-homomorphism of the homotopy groups in dimensions $\leq n-1$.

Define $\mathcal{C}_{i}=\left\{C \in \mathcal{C}: 1 / i+1<d_{C} \leq 1 / i\right\}$. Note that the closure of the union of the elements of $\mathcal{C}_{i}$ does not intersect $A$ since otherwise there is a point of $A$ to which a sequence of elements of $\mathcal{C}_{i}$ converges and therefore $\mathcal{C}_{i}$ would contain elements $C$ with arbitrarily small $d_{C}$ because $X$ is locally $(n-1)$-connected.

Let $\mathcal{U}$ be an open cover of $X \backslash A$ such that $\mathcal{U}$ properly approaches $A$ and $\mathcal{U}$ refines $\mathcal{W}$. Then for every $i$ we can approximate the identity map of $X$ by a closed embedding $e_{i}: X \rightarrow X$ such that $e_{i}(X) \subset X \backslash A, e_{i}$ is $\mathcal{W}$-close to the identity map of $X$ and, for every $C \in \mathcal{C}_{i}, \operatorname{diam}\left(e_{i}\left(G_{C}\right)\right) \leq \operatorname{diam}\left(G_{C}\right)+$ $1 / i, e_{i}(C) \subset \operatorname{st}(C, \mathcal{U})$ and every map $f: S^{p} \rightarrow C$ from a $p$-dimensional sphere $S^{p}, p \leq n-1$, can be homotoped into $e_{i}(C)$ inside $\operatorname{st}(C, \mathcal{U})$. Write $Y_{C}=\operatorname{st}(C, \mathcal{U}) \cup e_{i}\left(G_{C}\right)$ for $C \in \mathcal{C}_{i}$. Clearly, the inclusion $C \subset Y_{C}$ induces the zero-homomorphism of the homotopy groups in dimensions $\leq n-1$.

Define $\mathcal{G}_{i}=\left\{e_{i}\left(G_{C}\right): C \in \mathcal{C}_{i}\right\}$ and $\mathcal{G}=\bigcup\left\{\mathcal{G}_{i}: i=1,2, \ldots\right\}$. Recall that $\operatorname{diam}\left(e_{i}\left(G_{C}\right)\right) \leq \operatorname{diam}\left(G_{C}\right)+1 / i \leq 3 / i$ for $C \in \mathcal{C}_{i}$. Then, since the elements of $\mathcal{G}_{i}$ are contained in the closed subset $e_{i}(X)$ which does not meet $A$, we can conclude that the collection $\mathcal{G}$ properly approaches $A$. Since each $e_{i}$ is 
$\mathcal{W}$-close to the identity map of $X$ and each $G_{C}$ is contained in an element of $\mathcal{W}$, we deduce that $\mathcal{G}$ refines st $\mathcal{W}$.

Clearly, the cover $\mathcal{Y}=\left\{Y_{C}: C \in \mathcal{C}\right\}$ of $X \backslash A$ refines $\operatorname{st}(\operatorname{st}(\mathcal{C}, \mathcal{U}), \mathcal{G})$. Then $\mathcal{Y}$ refines $\operatorname{st}^{2} \mathcal{W}$ and, since $\mathcal{C}, \mathcal{U}$ and $\mathcal{G}$ properly approach $A$, we see that $\mathcal{Y}$ properly approaches $A$ as well. Set $V_{C}=\operatorname{st}\left(Y_{C}, \mathcal{U}\right)$. Then $\mathcal{V}=\left\{V_{C}: C \in \mathcal{C}\right\}$ properly approaches $A, \mathcal{V}$ refines $\mathrm{st}^{3} \mathcal{W}$ and therefore $\mathcal{V}$ has all the required properties.

In the proof of Proposition 3.2 we will use the following notion of maps witnessing intersections. Let $\mathcal{P}$ be a partition of an $m$-dimensional manifold $M$. Let $P_{0}, \ldots, P_{t}$ be distinct elements of $\mathcal{P}$ and let $P=P_{0} \cap \cdots \cap P_{t}$ be the intersection of $\mathcal{P}$. Denote by $\Delta_{P}$ a $t$-dimensional simplex with vertices $v_{0}, \ldots, v_{t}$. A map $e_{P}: \Delta_{P} \rightarrow M_{1}^{\prime}$ is called a map witnessing the intersection $P$ if $e_{P}\left(\Delta_{P}\right) \subset \operatorname{Int}\left(P_{0} \cup \cdots \cup P_{t}\right), e_{P}\left(v_{i}\right) \in \operatorname{Int} P_{i}$ and $P_{i}$ does not intersect $e_{P}\left(\Delta_{P}^{i}\right)$, where $\Delta_{P}^{i}$ is the face of $\Delta_{P}$ spanned by the vertices $\left\{v_{0}, \ldots, v_{t}\right\} \backslash\left\{v_{i}\right\}, i=0, \ldots, t$. It is clear that any sufficiently close approximation of a map witnessing the intersection $P$ also witnesses the intersection $P$.

Note that if there is a map $e_{P}$ witnessing the intersection $P$ then $P \neq \emptyset$. Indeed, aiming at a contradiction assume that $P=\emptyset$. Enlarge each $e_{P}^{-1}\left(P_{i}\right)$ to an open subset $e_{P}^{-1}\left(P_{i}\right) \subset G_{i}$ of $\Delta_{P}$ such that $G_{i}$ does not meet $\Delta_{P}^{i}$ and $G_{0} \cap \cdots \cap G_{t}=\emptyset$. Let $f_{0}, f_{1}, \ldots, f_{t}$ be a partition of unity subordinated to the cover $\mathcal{G}=\left\{G_{0}, \ldots, G_{t}\right\}$ of $\Delta_{P}$. Define $f: \Delta_{P} \rightarrow \Delta_{P}$ by $f(x)=$ $f_{0}(x) v_{0}+\cdots+f_{t}(x) v_{t}, x \in \Delta_{P}$. Then $f\left(\Delta_{P}\right) \subset \partial \Delta_{P}$ and, for every $i$ and $x \in \Delta_{P}^{i}, f(x) \in \Delta_{P}^{i}$. Hence $f$ restricted to $\partial \Delta_{P}$ is homotopic to the identity map of $\partial \Delta_{P}$ and this contradicts the fact that $\partial \Delta_{P}$ is not a retract of $\Delta_{P}$.

Assume that $P \neq \emptyset$ and let us show how to construct a map $e_{P}: \Delta_{P} \rightarrow M$ which witnesses the intersection $P$. Fix a point $x_{P} \in \operatorname{Int} P$ and a neighborhood $W$ of $x_{P}$ in $M$ such that $W \subset \operatorname{Int}\left(P_{0} \cup \cdots \cup P_{t}\right)$. Replacing $W$ by a smaller neighborhood of $x_{P}$ we may assume that for every intersection $P^{\prime}$ of $\mathcal{P}$ containing $x_{P}, P^{\prime} \cap W$ is contractible.

Let $F_{i}=$ the star of $v_{i}$ with respect to the first barycentric subdivision of $\Delta_{P}$. Clearly, $\mathcal{F}=\left\{F_{0}, \ldots, F_{t}\right\}$ is a partition of $\Delta_{P}$. Let us say that a map $e_{P}: \Delta_{P} \rightarrow W$ preserves the intersection $F^{\prime}=F_{i_{0}} \cap \cdots \cap F_{i_{j}}$ if $F^{\prime}=e_{P}^{-1}\left(P_{i_{0}} \cap \cdots \cap P_{i_{j}}\right)$. It is easy to see that a map preserving all the intersections of $\mathcal{F}$ witnesses the intersection $P$.

A map $e_{P}$ preserving all the intersections of $\mathcal{F}$ can be constructed as follows. First send the barycenter of $\Delta_{P}$ to $x_{P}$. Assume that we already constructed $e_{P}$ on the union $C_{j}$ of the finite intersections of $\mathcal{F}$ of dimension $\leq j$ such that $e_{P}$ preserves the intersections of $\mathcal{F}$ of dimension $\leq j$. Take a $(j+1)$-dimensional intersection $F^{\prime}=F_{i_{0}} \cap \cdots \cap F_{i_{j+1}}$ of $\mathcal{F}$ and let $P^{\prime}=$ $P_{i_{0}} \cap \cdots \cap P_{i_{j+1}}$ be the corresponding intersection of $\mathcal{P}$. Then $e_{P}\left(C_{j} \cap F^{\prime}\right) \subset$ 
$\partial\left(P^{\prime} \cap W\right)$ and, since $P^{\prime} \cap W$ is contractible, we can extend $e_{P}$ over $F^{\prime}$ so that $e_{P}\left(F^{\prime} \backslash C_{j}\right) \subset \operatorname{Int} P^{\prime} \cap W$. Thus we construct the desired map $e_{P}: \Delta_{P} \rightarrow W$.

Proof of Proposition 3.2. We only need to prove the second part of the proposition because the first part follows from the second one if we assume that $\Gamma$ is a singleton. Let $\psi_{i}: X_{i} \rightarrow \Gamma$ be $U V^{n-1}$-maps, $\mathcal{O}^{+}$an open cover of $\Gamma$ and $\omega$ a positive integer which depends only on $n$ and will be determined later. Then there is a sequence $\mathcal{O}^{0}, \mathcal{O}^{1}, \ldots, \mathcal{O}^{\omega}=\mathcal{O}^{+}$of open covers of $\Gamma$ such that for the open covers $\mathcal{O}_{i}^{j}=\psi_{i}^{-1}\left(\mathcal{O}^{j}\right)$ of $X_{i}$ we have st $\mathcal{O}_{i}^{j} \prec_{n-1} \mathcal{O}_{i}^{j+1}$ for $i=1,2$ and $j=0,1, \ldots, \omega-1$.

Apply Proposition 3.3 for $X, \psi$ and $\mathcal{U}^{+}$replaced by $X_{2}, \psi_{2}$ and $\mathcal{O}^{0}$ respectively in order to get an open cover $\mathcal{O}^{-1}$ of $\Gamma$ which corresponds to $\mathcal{U}^{-}$. Once again apply Proposition 3.3 for $X, \psi$ and $\mathcal{U}^{+}$replaced by $X_{1}$, $\psi_{1}$ and $\mathcal{O}^{-1}$ respectively in order to get an open cover $\mathcal{O}^{-2}$ of $\Gamma$ which corresponds to $\mathcal{U}^{-}$. Clearly, we can assume that $\mathcal{O}^{-2}$ refines $\mathcal{O}^{-1}$ and that $\mathcal{O}^{-1}$ refines $\mathcal{O}^{0}$.

Set $\mathcal{O}^{-}=\mathcal{O}^{-2}, \mathcal{O}_{i}^{j}=\psi_{i}^{-1}\left(\mathcal{O}^{j}\right)$ for $i=1,2$ and $j=-1,-2$ and assume that $\psi_{1} \mid A$ and $\psi_{2} \circ f_{A}$ are $\mathcal{O}^{-}$-close. The proof of the proposition splits into two independent parts.

Constructing an initial partition $\mathcal{P}_{2}$. Let an open subset $M_{1}^{\prime}$ of $M_{1}$ and a rational partition $\mathcal{P}_{1}$ of $M_{1}^{\prime}$ satisfy the assumptions of the proposition. In each non-empty finite intersection $P$ of $\mathcal{P}$ fix a point $x_{P} \in \operatorname{Int} P$ such that $x_{P} \in P\left(k_{1}\right)$ and take $e_{P}: \Delta_{P} \rightarrow M_{1}^{\prime}$ witnessing the intersection $P$ with $e_{P}\left(\Delta_{P}\right)$ so close to $x_{P}$ that the collection of the images of $e_{P}\left(\Delta_{P}\right)$ for all non-empty finite intersections of $\mathcal{P}_{1}$ will form a discrete family in $M_{1}^{\prime}$. Replace each $e_{P}$ by a sufficiently close $Z$-embedding into $M_{1}\left(k_{1}\right)=X_{1} \backslash A_{1}$ preserving the other properties of $e_{P}$. Denote by $Z_{1}$ the union of $A_{1}$ with the union of the images of all the maps $e_{P}$. Then $Z_{1}$ is a $Z$-set in $X_{1}$.

Assume that $\psi_{1} \mid A_{1}$ and $\psi_{2} \circ f_{A}$ are $\mathcal{O}^{-}$-close and $\mathcal{P}_{1}$ refines the extension of $\psi_{1}^{-1}\left(\mathcal{O}^{-}\right)$to $Y_{1}$. By Proposition 3.3 extend $f_{A}: A_{1} \rightarrow A_{2}$ to a $Z$-embedding $g_{1}: X_{1} \rightarrow X_{2}$ such that $\psi_{1}$ and $\psi_{2} \circ g_{1}$ are $\mathcal{O}^{-1}$-close. Once again by Proposition 3.3 extend the map $\left.g_{1}^{-1}\right|_{\ldots}: Z_{2}=g_{1}\left(Z_{1}\right) \rightarrow Z_{1}$ to a $Z$-embedding $g_{2}: X_{2} \rightarrow X_{1}$ such that $\psi_{2}$ and $\psi_{1} \circ g_{2}$ are $\mathcal{O}^{0}$-close.

Take an open cover $\mathcal{U}_{1}$ of $X_{1} \backslash A_{1}$ having the following properties:

(1) $\mathcal{U}_{1}$ properly approaches $A_{1}$ and refines $\mathcal{O}_{1}^{0}$;

(2) for every non-empty intersection $P=P_{0} \cap \cdots \cap P_{t}$ of distinct elements $P_{0}, \ldots, P_{t}$ of $\mathcal{P}_{1}$ and the map $e_{P}: \Delta_{P}=\left[v_{0}, \ldots, v_{t}\right] \rightarrow M_{1}\left(k_{1}\right) \subset M_{2}^{\prime}$ we have $\operatorname{st}\left(e_{P}\left(\Delta_{P}\right), \mathcal{U}_{1}\right) \subset \operatorname{Int}\left(P_{0} \cup \cdots \cup P_{t}\right)$, and for every $P_{i}$ we have $\operatorname{st}\left(P_{i}, \mathcal{U}_{1}\right) \cap \operatorname{st}\left(e_{P}\left(\Delta_{P}^{i}\right), \mathcal{U}_{1}\right)=\emptyset$ and $\operatorname{st}\left(e_{P}\left(v_{i}\right), \mathcal{U}_{1}\right) \subset \operatorname{Int} P_{i}$;

(3) for any finite intersections $P$ and $P^{\prime}$ of $\mathcal{P}_{1}$ such that $P \cap P^{\prime}=\emptyset$ we have $\operatorname{st}\left(P, \mathcal{U}_{1}\right) \cap \operatorname{st}\left(P^{\prime}, \mathcal{U}_{1}\right)=\emptyset$. 
Define $\mathcal{U}_{2}=g_{2}^{-1}\left(\mathcal{U}_{1}\right)$. Since $g_{2}$ is a closed embedding extending $f_{A}^{-1}$ and $\mathcal{U}_{1}$ properly approaches $A_{1}$, we find that $\mathcal{U}_{2}$ properly approaches $A_{2}$. Since $\psi_{2}$ and $\psi_{1} \circ g_{2}$ are $\mathcal{O}^{0}$-close and $\mathcal{U}_{1}$ refines $\mathcal{O}_{1}^{0}$, we see that $\mathcal{U}_{2}$ refines $\mathcal{O}_{2}^{1}$. Let $\mathcal{U}_{2}^{M}$ be the extension of $\mathcal{U}_{2}$ to $M_{2}$ and let $M_{2}^{\prime}$ be the union of the elements of $\mathcal{U}_{2}^{M}$. Note that $M_{2}^{\prime}$ is $(n-1)$-connected $\left(=l_{i}\right.$-co-connected) since $M_{2}\left(k_{2}\right) \subset M_{2}^{\prime}$. Take a rational triangulation of $M_{2}^{\prime}$ such that, for the partition $\mathcal{B}$ of $M_{2}^{\prime}$ formed by the stars of the vertices with respect to the first barycentric subdivision of the triangulation, $\mathcal{B}$ refines $\mathcal{U}_{2}^{M}$.

Consider $g_{2}$ as a map into $Y_{1}$. Arrange the elements of $\mathcal{P}_{1}=\left\{P^{1}, P^{2}, \ldots\right\}$ into a sequence and define $\mu\left(P^{1}\right)=$ the union of the elements of $\mathcal{B}$ that intersect $g_{2}^{-1}\left(P^{1}\right), \mu\left(P^{2}\right)=$ the union of the elements of $\mathcal{B}$ that intersect $g_{2}^{-1}\left(P^{2}\right)$ but do not intersect $g_{2}^{-1}\left(P^{1}\right), \mu\left(P^{3}\right)=$ the union of the elements of $\mathcal{B}$ that intersect $g_{2}^{-1}\left(P^{3}\right)$ but do not intersect $g_{2}^{-1}\left(P^{1}\right) \cup g_{2}^{-1}\left(P^{2}\right)$ and so on. Let $\mathcal{P}_{2}=\left\{\mu\left(P^{1}\right), \mu\left(P^{2}\right), \ldots\right\}$. Since each element of $\mathcal{B}$ intersects $M_{2}\left(k_{2}\right)$ we see that $\mathcal{P}_{2}$ covers $M_{2}^{\prime}$. The property (2) guarantees that for every $P \in \mathcal{P}_{1}, g_{1}\left(e_{P}\left(\Delta_{P}\right)\right) \subset \mu(P)$ and therefore $\mu(P) \neq \emptyset$. Then, by $2.2, \mathcal{P}_{2}$ is a partition of $M_{2}^{\prime}$. The property (2) also guarantees that for every nonempty intersection $P=P_{0} \cap \cdots \cap P_{t}$ of distinct elements $P_{0}, \ldots, P_{t} \in \mathcal{P}_{1}$, the map $g_{1} \circ e_{P}$ witnesses the intersection of $\mu\left(P_{0}\right), \ldots, \mu\left(P_{t}\right)$ and therefore $\mu\left(P_{0}\right) \cap \cdots \cap \mu\left(P_{t}\right) \neq \emptyset$. The property (3) implies that we do not create additional intersections in $\mathcal{P}_{2}$ and hence $\mu: \mathcal{P}_{1} \rightarrow \mathcal{P}_{2}$ is a matching of partitions. It follows from the construction that

$$
\text { (4) } \mu(P) \subset \operatorname{st}\left(g_{2}^{-1}(P), \mathcal{B}\right) \subset \operatorname{st}\left(g_{2}^{-1}(P), \mathcal{U}_{2}^{M}\right) \text { for every } P \in \mathcal{P}_{1} \text {. }
$$

Since $\mathcal{P}_{1}$ restricted to $X_{1} \backslash A_{1}$ refines $\mathcal{O}_{1}^{0}$ and the maps $\psi_{2}$ and $\psi_{1} \circ g_{2}$ are $\mathcal{O}^{0}$-close, we find that $g_{2}^{-1}\left(\mathcal{P}_{1}\right)$ refines $\mathcal{O}_{2}^{1}$. Then, since $\mathcal{B}$ refines $\mathcal{U}_{2}^{M}$ and $\mathcal{U}_{2}$ refines $\mathcal{O}_{2}^{1}$, we deduce by (4) that $\mathcal{P}_{2}$ refines the extension of $\mathcal{O}_{2}^{2}$ to $Y_{2}$ and $\mu, \psi_{1}$ and $\psi_{2}$ agree with respect to $\mathcal{O}^{2}$.

Since $g_{2}$ is a closed embedding which coincides with $f_{A}^{-1}$ on $A_{2}$ and $g_{2}\left(X_{2}\right)$ intersects all the elements of $\mathcal{P}_{1}$, we conclude that $g_{2}^{-1}\left(\mathcal{P}_{1}\right)$ properly approaches $A_{2}$ and the correspondence between $\mathcal{P}_{1}$ and $g_{2}^{-1}\left(\mathcal{P}_{1}\right)$ defined by $P \mapsto g_{2}^{-1}(P), P \in \mathcal{P}_{1}$, agrees with $f_{A}$. Since $\mathcal{U}_{2}$ properly approaches $A_{2}$, we see that $\mathcal{U}_{2}^{M}$ properly approaches $A_{2}$ as well. Then it is easy to derive from (4) that $\mathcal{P}_{2}$ properly approaches $A_{2}$ and $\mu$ agrees with $f_{A}$. Recall that $\mathcal{P}_{2}$ refines the extension of $\mathcal{O}_{2}^{2}$ to $Y_{2}$ and $\mu, \psi_{1}$ and $\psi_{2}$ agree with respect to $\mathcal{O}^{2}$.

Improving connectivity of $\mathcal{P}_{2}$. Without loss of generality we may replace $M_{1}$ and $M_{2}$ by $M_{1}^{\prime}$ and $M_{2}^{\prime}$ respectively and assume that $M_{1}=M_{1}^{\prime}$ and $M_{2}=M_{2}^{\prime}$ for the partition $\mathcal{P}_{1}$ and the initial partition $\mathcal{P}_{2}$. Let us show that using 2.7 we can modify $\mathcal{P}_{2}$ into the required $l_{2}$-co-connected rational partition. The construction of 2.7 is a combination of 2.5 and 2.6, and 2.5 consists of 2.4 applied finitely many times. Abusing the notation we always denote by $M_{2}, F$ and $\mathcal{P}_{2}$ the manifold, the PL-subcomplex of $M_{2}$ and the 
decomposition of $M_{2}$ which are the input of 2.4 and 2.6 (= the output of the previous applications of 2.4 and 2.6), and by $M_{2}^{\prime}, F^{\prime}$ and $\mathcal{P}_{2}^{\prime}$ the output of 2.4 and 2.6 (= the modifications of $M_{2}, F$ and $\mathcal{P}_{2}$ that are obtained after applying 2.4 and 2.6), and we denote again by $\mu$ the correspondence $\mu: \mathcal{P}_{1} \rightarrow \mathcal{P}_{2}^{\prime}$ which is the composition of $\mu: \mathcal{P}_{1} \rightarrow \mathcal{P}_{2}$ with the natural one-to-one correspondence between $\mathcal{P}_{2}$ and its modification $\mathcal{P}_{2}^{\prime}$ (defined by sending each element of $\mathcal{P}_{2}$ to its modification). Thus we start with the initial partition $\mathcal{P}_{2}$ that during 2.7 turns into a decomposition forming a partition on $U=M_{2} \backslash F$ and, gradually reducing the dimension of $F, \mathcal{P}_{2}$ returns to be a partition at the end of 2.7. Recall that after applying 2.4 and 2.6 we always obtain an open subset $M_{2}^{\prime}$ of $M_{2}$ such that $R=M_{2} \backslash M_{2}^{\prime}$ is a PL-presented subset of $M_{2}$ with $\operatorname{dim} R \leq n=m_{2}-l_{2}+1 \leq k_{2}$. By 2.8 we can choose a homeomorphism of $h: A_{2} \cup M_{2} \rightarrow A_{2} \cup M_{2}$ such that $h$ is arbitrarily close to the identity map, $h(a)=a$ for $a \in A_{2}, h(R)$ is a rationally presented closed subset of $M_{2}, h\left(F^{\prime}\right)$ is a rational subcomplex of $h\left(M_{2}^{\prime}\right)$, $h\left(\mathcal{P}_{2}^{\prime}\right)$ is a rational decomposition of $h\left(M_{2}^{\prime}\right)$ and $h$ restricted to $M_{2} \backslash R$ is a PL-homeomorphism to $M_{2} \backslash h(R)$. Then replacing $M_{2}^{\prime}, F^{\prime}$ and $\mathcal{P}_{2}^{\prime}$ by $h\left(M_{2}^{\prime}\right)$, $h\left(F^{\prime}\right)$ and $h\left(\mathcal{P}_{2}^{\prime}\right)$ respectively we can always assume that $M_{2}\left(k_{2}\right) \subset M_{2}^{\prime}, F^{\prime}$ is a rational subcomplex of $M_{2}^{\prime}$ and $\mathcal{P}_{2}^{\prime}$ is a rational decomposition. Thus at the end of 2.7 we deduce that $\operatorname{dim} F^{\prime} \leq n=m_{2}-l_{2}+1 \leq k_{2}$ and hence $M_{2}\left(k_{2}\right) \subset M_{2}^{\prime} \backslash F^{\prime}$. Now we can replace the final modification $M_{2}^{\prime}$ of $M_{2}$ by $M_{2}^{\prime} \backslash F$ and get the final partition on $M_{2}^{\prime}$.

We will apply 2.4 and 2.6 so that there will exist an open cover $\mathcal{W}$ of $M_{2}$ such that $\mathcal{W}$ properly approaches $A_{2}$ and for every $P \in \mathcal{P}_{2}$ and its modification $P^{\prime} \in \mathcal{P}_{2}$ we have $P^{\prime} \subset \operatorname{st}\left(P, \mathrm{st}^{2} \mathcal{W}\right)$. This property implies that $\mathcal{P}_{2}^{\prime}$ will properly approach $A_{2}$ and $\mu: \mathcal{P}_{1} \rightarrow \mathcal{P}_{2}^{\prime}$ will agree with $f_{A}$. In addition, we will apply 2.4 and 2.6 so that there exists $j$ such that the modification $\mathcal{P}_{2}^{\prime}$ of $\mathcal{P}_{2}$ will refine the extension of $\mathcal{O}_{2}^{j}$ to $Y_{2}$ and $\mu: \mathcal{P}_{1} \rightarrow \mathcal{P}_{2}^{\prime}$, $\psi_{1}$ and $\psi_{2}$ will agree with respect to $\mathcal{O}^{j}$. Clearly, applying 2.8 as described above, we preserve the properties that $\mathcal{P}_{2}^{\prime}$ properly approaches $A_{2}$ and $\mu$ agrees with $f_{A}$, and choosing the homeomorphism $h$ sufficiently close to the identity map of $M_{2}^{\prime}$ we can increase $j$ by 1 and assume that $\mathcal{P}_{2}^{\prime}$ refines the extension of $\mathcal{O}_{2}^{j}$ to $Y_{2}$ and $\mu, \psi_{1}$ and $\psi_{2}$ agree with respect to $\mathcal{O}^{j}$.

Let us first analyze 2.4. Assume that $\mathcal{P}_{2}$ properly approaches $A_{2}, \mathcal{P}_{2}$ refines the extension of $\mathcal{O}_{2}^{j}$ to $Y_{2}$ and $\mu, \psi_{1}$ and $\psi_{2}$ agree with respect to $\mathcal{O}^{j}$. Take any open cover $\mathcal{U}$ of $M_{2}$ such that $\mathcal{U}$ refines the extension of $\mathcal{O}_{2}^{j}$ to $Y_{2}$ and $\mathcal{U}$ properly approaches $A_{2}$.

Set $\mathcal{C}_{M}=\operatorname{st}\left(\operatorname{st} \mathcal{P}_{2}, \mathcal{U}\right)$. Then $\mathcal{C}_{M}$ is an open cover of $M_{2}$ such that $\mathcal{C}_{M}$ properly approaches $A_{2}$, st $\mathcal{P}_{2}$ refines $\mathcal{C}_{M}$ and $\mathcal{C}_{M}$ refines the extension of $\mathcal{O}_{2}^{j+2}$ to $Y_{2}$. Let $\mathcal{C}=\mathcal{C}_{M} \mid M_{2}\left(k_{2}\right)$. Recall that $\mathcal{O}_{2}^{j+2} \prec_{n-1} \mathcal{O}_{2}^{j+3}$. Then by Proposition 3.4 there is an open cover $\mathcal{V}$ of $M_{2}\left(k_{2}\right)$ such that $\mathcal{V}$ properly approaches $A_{2}, \mathcal{C} \prec_{n-1} \mathcal{V}, \mathcal{V}$ refines $\operatorname{st}^{3} \mathcal{O}_{2}^{j+3}$ and hence $\mathcal{V}$ refines $\mathcal{O}_{2}^{j+6}$. Let 
$\mathcal{W}=\operatorname{st}(\mathcal{V}, \mathcal{U})$. Note that $\mathcal{W}$ is an open cover of $M_{2}, \mathcal{W}$ properly approaches $A_{2}$ and refines the extension of $\mathcal{O}_{2}^{j+7}$ to $Y_{2}$. Since every map from a sphere of dimension $\leq n-1$ to an open subset $G$ of $M_{2}$ can be homotoped inside $G$ to a map into $G \cap M_{2}\left(k_{2}\right)$, we conclude that $\mathcal{C} \prec_{n-1} \mathcal{V}$ implies $\mathcal{C}_{M} \prec_{n-1} \mathcal{W}$ and therefore st $\mathcal{P}_{2} \prec_{n-1} \mathcal{W}$. Then 2.4 can be carried out so that for every $P \in \mathcal{P}_{2}$ and its modification $P^{\prime} \in \mathcal{P}_{2}^{\prime}$ we have $P^{\prime} \subset \operatorname{st}\left(P, \mathrm{st}^{2} \mathcal{W}\right)$. The last property also implies that $\mathcal{P}_{2}^{\prime}$ refines the extension of $\mathcal{O}_{2}^{j+10}$ to $Y_{2}$ and $\mu: \mathcal{P}_{1} \rightarrow \mathcal{P}_{2}^{\prime}$, $\psi_{1}$ and $\psi_{2}$ agree with respect to $\mathcal{O}^{j+10}$.

Now we will analyze 2.6. Once again we assume that $\mathcal{P}_{2}$ properly approaches $A_{2}, \mathcal{P}_{2}$ refines the extension of $\mathcal{O}_{2}^{j}$ to $Y_{2}$ and $\mu, \psi_{1}$ and $\psi_{2}$ agree with respect to $\mathcal{O}^{j}$. Then the collection st $\mathcal{P}_{2}$ properly approaches $A_{2}$. Since for $P \in \mathcal{P}_{2}$, st $\left(P, \mathcal{P}_{2}\right)$ is a union of elements of $\mathcal{P}_{2}$, we can naturally define the set $\mu^{-1}\left(\operatorname{st}\left(P, \mathcal{P}_{2}\right)\right)$ as the union of the corresponding elements of $\mathcal{P}_{1}$. Then the collection $\mu^{-1}$ (st $\left.\mathcal{P}_{2}\right)$ properly approaches $A_{1}$ since $\mu$ agrees with $f_{A}$, and $\mu^{-1}\left(\right.$ st $\left.\mathcal{P}_{2}\right)$ refines the extension of $\mathcal{O}_{1}^{j+2}$ to $Y_{1}$ since $\mu, \psi_{1}$ and $\psi_{2}$ agree with respect to $\mathcal{O}^{j}$. Let $\mathcal{U}$ be an open cover of $M_{1}$ such that $\mathcal{U}$ refines the extension of $\mathcal{O}_{1}^{j+2}$ to $Y_{1}$ and $\mathcal{U}$ properly approaches $A_{1}$.

Set $\mathcal{C}_{M}=\operatorname{st}\left(\mu^{-1}\left(\right.\right.$ st $\left.\left.\mathcal{P}_{2}\right), \mathcal{U}\right)$. Then $\mathcal{C}_{M}$ is an open cover of $M_{1}$ such that $\mathcal{C}_{M}$ properly approaches $A_{1}, \mu^{-1}$ (st $\left.\mathcal{P}_{2}\right)$ refines $\mathcal{C}_{M}$ and $\mathcal{C}_{M}$ refines the extension of $\mathcal{O}_{1}^{j+3}$ to $Y_{1}$. Set $\mathcal{C}=\mathcal{C}_{M} \mid M_{1}\left(k_{1}\right)$. Recall that $\mathcal{O}_{1}^{j+3} \prec_{n-1} \mathcal{O}_{1}^{j+4}$. Then, by Proposition 3.4, there is an open cover $\mathcal{V}$ of $M_{1}\left(k_{1}\right)$ such that $\mathcal{V}$ properly approaches $A_{1}, \mu^{-1}\left(\right.$ st $\left.\mathcal{P}_{2}\right)$ restricted to $M_{1}\left(k_{1}\right)$ is an $(n-1)$-refinement of $\mathcal{V}$, $\mathcal{V}$ refines $\mathrm{st}^{3} \mathcal{O}_{1}^{j+4}$ and hence $\mathcal{V}$ refines $\mathcal{O}_{1}^{j+7}$.

Define $\mathcal{H}=\operatorname{st}\left(\mathcal{V}, \mu^{-1}\left(\right.\right.$ st $\left.\left.\mathcal{P}_{2}\right)\right)$. It is clear that $\mu^{-1}\left(\right.$ st $\left.\mathcal{P}_{2}\right)$ refines $\mathcal{H}$, $\mu^{-1}\left(\right.$ st $\left.\mathcal{P}_{2}\right)$ restricted to $M_{1}\left(k_{1}\right)$ is an $(n-1)$-refinement of $\mathcal{H}, \mathcal{H}$ properly approaches $A_{1}$ and $\mathcal{H}$ refines $\mathcal{O}_{1}^{j+8}$. Since $\mu^{-1}\left(\operatorname{st}\left(P, \mathcal{P}_{2}\right)\right)$ is a rational submanifold of $M_{1}$ of dimension $m_{1}$, every map from a space of dimension $\leq n-1$ into $\mu^{-1}\left(\operatorname{st}\left(P, \mathcal{P}_{2}\right)\right)$ can be homotoped inside $\mu^{-1}\left(\operatorname{st}\left(P, \mathcal{P}_{2}\right)\right)$ into $\mu^{-1}\left(\operatorname{st}\left(P, \mathcal{P}_{2}\right)\right) \cap M_{1}\left(k_{1}\right)$ and therefore $\mu^{-1}\left(\right.$ st $\left.\mathcal{P}_{2}\right)$ is an $(n-1)$-refinement of $\mathcal{H}$. Note that $\mu(\mathcal{H})$ is well-defined because each element of $\mathcal{H}$ is a union of elements of $\mathcal{P}_{1}$. Also, since $\mu, \psi_{1}$ and $\psi_{2}$ agree with respect to $\mathcal{O}^{j}$, we deduce that $\mu(\mathcal{H})$ refines the extension of $\mathcal{O}_{2}^{j+9}$ to $Y_{2}$.

Assume that $F$ is a PL-subcomplex of $M_{2}$ such that for $U=M_{2} \backslash F$ the decomposition $\mathcal{P}_{2}$ forms on $U$ an $l$-co-connected partition, $l \geq l_{2}$, for which $\mu: \mathcal{P}_{1} \rightarrow \mathcal{P}_{2}$ becomes a matching when $\mathcal{P}_{2}$ is restricted to $U$. Recall that $\mu^{-1}\left(\right.$ st $\left.\mathcal{P}_{2}\right)$ is an $(n-1)$-refinement of $\mathcal{H}$. Then, by 2.3 , st $\left(\mathcal{P}_{2}\right) \mid U$ is an $\left(m_{2}-l\right)$-refinement of $\mu(\mathcal{H}) \mid U$.

Take any open cover $\mathcal{G}$ of $M_{2}$ such that $\mathcal{G}$ properly approaches $A_{2}$ and $\mathcal{G}$ refines the extension of $\mathcal{O}_{2}^{j}$ to $Y_{2}$. Define $\mathcal{W}=\operatorname{st}(\mu(\mathcal{H}), \mathcal{G})$ and note that $\mathcal{W}$ refines the extension of $\mathcal{O}_{2}^{j+10}$ to $Y_{2}$. Then for every $P \in \mathcal{P}_{2}$ there are $H \in \mu(\mathcal{H})$ and $W \in \mathcal{W}$ with $\operatorname{st}\left(P, \mathcal{P}_{2}\right) \subset H \subset W$ and such that the inclusion 
st $\left(P, \mathcal{P}_{2}\right) \cap U \subset H \cap U$ induces the zero-homomorphism of the homotopy groups in dimensions $\leq m_{2}-l$. Hence 2.6 can be carried out so that for every $P \in \mathcal{P}_{2}$ and its modification $P^{\prime} \in \mathcal{P}_{2}^{\prime}$ we get $P^{\prime} \subset \operatorname{st}\left(P, \mathrm{st}^{2} \mathcal{W}\right)$. The last property also implies that $\mathcal{P}_{2}^{\prime}$ refines the extension of $\mathcal{O}_{2}^{j+13}$ to $Y_{2}$ and $\mu: \mathcal{P}_{1} \rightarrow \mathcal{P}_{2}^{\prime}, \psi_{1}$ and $\psi_{2}$ agree with respect to $\mathcal{O}^{j+13}$.

Now we need to determine the maximal possible value of $j$ which we assign to $\omega$ at the beginning of the proof. From the proof it is clear that this value depends only on the number of times we apply the constructions 2.4, 2.6, 2.8 and it is easy to see from 2.7 and 2.5 that this number depends only on $n$.

\section{Constructions of improving connectivity and absorbing simplexes}

4.1. A black hole modification. Let $B^{q}, B^{m-q}, B_{*}^{m-q}$ and $B^{m}=B^{q} \times$ $B^{m-q}$ be cubes with the dimensions indicated by the superscripts such that $B_{*}^{m-q}$ is contained in Int $B^{m-q}$ and the centers of $B_{*}^{m-q}$ and $B^{m-q}$ coincide (recall that we assume that the centers of the cubes are located at $O$ and we identify $B^{q}$ and $B^{m-q}$ with the subsets $B^{q} \times O$ and $O \times B^{m-q}$ of $B^{m}$ respectively; see 2.1). Define $T=B^{q} \times\left(B^{m-q} \backslash \operatorname{Int} B_{*}^{m-q}\right)$ and $S=\partial B^{m-q}$. Note that $S$ is an $(m-q-1)$-dimensional sphere in $\partial T$ and $T$ is a subset of $B^{m}$ which is PL-homeomorphic to the product $B^{q+1} \times S$, where $S$ is identified with $a \times S$ for a point $a \in \partial B^{q+1}$.

Assume that $B^{m}$ is PL-embedded in Int $M$ of a triangulated $m$-dimensional manifold $M$. Set $L=M \backslash \operatorname{Int} T$ and assume that $F_{L}$ is a PL-subcomplex of $L$ and $\mathcal{P}_{L}$ is a decomposition of $L$ such that $S \subset F_{L}$ and $\mathcal{P}_{L}$ restricted to $L \backslash F_{L}$ and to $\partial T \backslash F_{L}$ are partitions.

Fix a triangulation $\mathcal{T}$ of $M$ that underlies $S, F_{L}, L$ and the elements of $\mathcal{P}_{L}$, and denote by $\mathcal{A}$ the collection of subcomplexes of $L$ with respect to this triangulation. We are going to extend each $A \in \mathcal{A}$ to a PL-subcomplex $A \subset A^{\pi}$ of $M$ such that the following conditions are satisfied:

- $P_{L}^{\pi}=P_{L}$ for $P_{L} \in \mathcal{P}_{L}$ such that $P_{L} \cap \partial T=\emptyset$;

- $\mathcal{P}_{L}^{\pi}=\left\{P_{L}^{\pi}: P_{L} \in \mathcal{P}_{L}\right\}$ is a decomposition of $M$ which forms a partition on $M \backslash F_{L}^{\pi}$;

- $F_{L}^{\pi} \cap L=F_{L}$ and $\mathcal{P}_{L}^{\pi}$ restricted to $L \backslash F_{L}^{\pi}$ coincides with $\mathcal{P}_{L}$ restricted to $L \backslash F_{L}$;

- the correspondence between $\mathcal{P}_{L}$ and $\mathcal{P}_{L}^{\pi}$ defined by $P_{L} \mapsto P_{L}^{\pi}$ induces a matching of partitions when $\mathcal{P}_{L}$ and $\mathcal{P}_{L}^{\pi}$ are restricted to $L \backslash F_{L}$ and $M \backslash F_{L}^{\pi}$ respectively;

- for every intersection $P_{L}=P_{L}^{0} \cap \cdots \cap P_{L}^{j}$ of elements $P_{L}^{0}, \ldots, P_{L}^{j}$ of $\mathcal{P}_{L}, P_{L}^{\pi} \backslash F_{L}^{\pi}=\left(\left(P_{L}^{0}\right)^{\pi} \cap \cdots \cap\left(P_{L}^{j}\right)^{\pi}\right) \backslash F_{L}^{\pi}$ and $P_{L} \backslash F_{L}$ is a (strong) deformation retract of $P_{L}^{\pi} \backslash F_{L}^{\pi}$; 
- $\operatorname{dim}\left(F_{L}^{\pi} \cap T\right) \backslash S \leq \operatorname{dim}\left(F_{L} \cap \partial T\right) \backslash S+1$;

- $F_{L}^{\pi}=F_{L}$ if $F_{L} \cap \partial T=S$.

Recall that $T$ is homeomorphic to the product $B^{q+1} \times S$, where $S$ is identified with $a \times S$ for a point $a \in \partial B^{q+1}$. Note that $B^{q+1} \backslash a$ is homeomorphic to $\left(\partial B^{q+1} \backslash a\right) \times[0,1)$ and hence $T \backslash S$ can be represented as the product $(\partial T \backslash S) \times[0,1)$, where $\partial T \backslash S$ corresponds to $(\partial T \backslash S) \times 0$. One of the ways to construct $A^{\pi}$ is to find a retraction $\pi: T \backslash S \rightarrow \partial T \backslash S$ which is topologically equivalent to the projection of $(\partial T \backslash S) \times[0,1)$ to $(\partial T \backslash S) \times 0$ such that, for $A^{\pi}$ defined by $A^{\pi}=A$ if $A \cap \partial T=\emptyset$ and $A^{\pi}=A \cup S \cup \pi^{-1}(A \cap \partial T)$ otherwise, $A^{\pi}$ is a PL-subcomplex of $M$ for every $A \in \mathcal{A}$. Such a retraction $\pi$ can be constructed, but the author could not find an elementary and direct argument for this. Therefore, for the sake of elementariness (and at the expense of a geometric transparency), we adopt a slightly different approach for constructing $A^{\pi}$ which is presented in 5.1. Note that all the properties of $\mathcal{P}_{L}^{\pi}$ and $F_{L}^{\pi}$ that will be used subsequently are described above. Thus for understanding the rest of the paper the reader may assume that the construction is carried out on the base of an appropriate retraction $\pi$. Also note that $S$ is added to $A^{\pi}$ for $A$ intersecting $\partial T$ in order to make $A^{\pi}$ a closed subset of $M$ and $\mathcal{P}_{L}^{\pi}$ a cover of $M$.

We will call the decomposition $\mathcal{P}_{L}^{\pi}$ a black hole modification of $\mathcal{P}_{L}$ with the sphere $S$ as the black hole of the modification.

4.2. Improving connectivity of an intersection. Assume that $m \geq 2 q+1$ and $l=m-q+2, M$ is a triangulated $m$-dimensional $(q-1)$-connected $(=(l-1)$-co-connected) manifold and $F$ is a PL-subcomplex of $M$ lying in Int $M$. Let $\mathcal{P}$ be a decomposition of $M$ which forms an $l$-co-connected partition on $U=M \backslash F$ and let $P=P_{0} \cap P_{1} \cap \cdots \cap P_{t}, 0 \leq t \leq m-l+1$, be an intersection of distinct elements $P_{0}, \ldots, P_{t}$ of $\mathcal{P}$ such that $P \cap U \neq \emptyset$. Then $P \cap U$ is an $(m-t)$-dimensional and $(q-t-2)$-connected (=l-co-connected) manifold.

Assume that the intersections of $\mathcal{P} \mid U$ of dimension $>m-t$ are $(l-1)$-coconnected. Let us show how to improve the connectivity of $P \cap U$ preserving the level of connectivity of the rest of the intersections.

Let $f: S_{P} \rightarrow \operatorname{Int}(P \cap U)$ be a PL-embedding of a triangulated $(q-t-1)$ dimensional sphere $S_{P}$ such that $f$ is not null-homotopic in $P \cap U$.

Consider a $t$-dimensional simplex $\Delta$ with vertices $V(\Delta)=\left\{v_{0}, \ldots, v_{t}\right\}$. Let $\Delta^{\prime}$ be a (sub)simplex of $\Delta$ lying in $\partial \Delta$ and let $V\left(\Delta^{\prime}\right) \subset V(\Delta)$ be the set of vertices of $\Delta^{\prime}$. Define $P\left(\Delta^{\prime}\right)=\bigcap\left\{P_{i}: v_{i} \in V(\Delta) \backslash V\left(\Delta^{\prime}\right)\right\}$. Note that $P(\emptyset)=P$ and $P\left(\Delta^{\prime}\right) \subset P\left(\Delta^{\prime \prime}\right)$ if $\Delta^{\prime} \subset \Delta^{\prime \prime}$.

For every simplex $\Delta^{\prime}$ of $\partial \Delta$ we will define by induction on $\operatorname{dim} \Delta^{\prime}$ a PL-embedding $f_{\Delta^{\prime}}: S_{P} * \Delta^{\prime} \rightarrow P\left(\Delta^{\prime}\right) \cap U$ such that $f_{\Delta^{\prime \prime}}$ extends $f_{\Delta^{\prime}}$ if $\Delta^{\prime} \subset \Delta^{\prime \prime}$. Setting $f_{\emptyset}=f$ we are done for $t=0$. Let $t>0$. Take a simplex 
$\Delta^{\prime}$ of dimension $i+1 \leq t-1$ and assume that $f_{\Delta^{\prime}}$ is defined for all $\Delta^{\prime}$ of dimension $\leq i$. Then the maps $f_{\Delta^{\prime \prime}}$ for $\Delta^{\prime \prime} \subset \partial \Delta^{\prime}$ define the corresponding PL-embedding $f_{\partial \Delta^{\prime}}: S_{P} * \partial \Delta^{\prime} \rightarrow \partial\left(P\left(\Delta^{\prime}\right) \cap U\right)$. Note that $S_{P} * \partial \Delta^{\prime}$ is a $(q-t+i)$-dimensional sphere and $P\left(\Delta^{\prime}\right) \cap U$ is $(m-t+i+2)$-dimensional and $(q-t+i+1)$-connected $(=(l-1)$-co-connected $)$ and $\operatorname{dim}\left(P\left(\Delta^{\prime}\right) \cap U\right)=$ $m-t+i+2 \geq 2 q+1-t+i+2 \geq 2(q-t+i+1)+1+(t-i-2) \geq 2(q-t+i+1)+1$ since $m \geq 2 q+1$ and $t-i-2 \geq 0$. Then $f_{\partial \Delta^{\prime}}$ can be extended to a PLembedding $f_{\Delta^{\prime}}: S_{P} * \Delta^{\prime} \rightarrow P\left(\Delta^{\prime}\right) \cap U$ such that $f_{\Delta^{\prime}}$ sends $\operatorname{Int}\left(S_{P} * \Delta^{\prime}\right)$ to $\operatorname{Int}\left(P\left(\Delta^{\prime}\right) \cap U\right)$.

The maps $f_{\Delta^{\prime}}$ define the PL-embedding $f_{\partial \Delta}$ of $S_{P} * \partial \Delta$ to $\operatorname{Int}\left(\left(P_{0} \cup \cdots \cup P_{t}\right)\right.$ $\cap U)$. Identify the $(q-1)$-dimensional sphere $S^{q-1}=S_{P} * \partial \Delta$ with its image under $f_{\partial \Delta}$ and, by 5.2 below, extend the embedding of $S^{q-1}$ to a PL-embedding of a $q$-dimensional cube $B^{q}$ such that $S^{q-1}=\partial B^{q}$ and the embedding of $B^{q}$ extends to a PL-embedding of an $m$-dimensional cube $B^{m}=B^{q} \times B^{m-q} \subset$ Int $M$ having the following properties: $P^{\prime} \cap B^{m}=$ $\left(B^{q} \cap P^{\prime}\right) \times B^{m-q}$ for every $P^{\prime} \in \mathcal{P} ; F \cap B^{m}=\left(B^{q} \cap F\right) \times B^{m-q} ; \mathcal{P}$ restricted to $N \cap U$ and to $\partial B^{m} \cap U$ are partitions, where $N=M \backslash \operatorname{Int} B^{m}$ and for every finite intersection $P^{\prime}$ of $\mathcal{P}$ such that $P^{\prime} \cap U \neq \emptyset$ we have $P^{\prime} \cap N \cap U \neq \emptyset$ and $P^{\prime} \cap N \cap U$ is a deformation retract of $\left(P^{\prime} \cap U\right) \backslash \operatorname{Int} B^{q}$. Note that for every finite intersection $P^{\prime}$ of $\mathcal{P}$ that intersects $U,\left(\operatorname{dim}\left(P^{\prime} \cap U\right)-(l-1)+1\right)$ $+q+1=\operatorname{dim}\left(P^{\prime} \cap U\right)-m+2 q+1 \leq \operatorname{dim}\left(P^{\prime} \cap U\right)$ and therefore the inclusion $\left(P^{\prime} \cap U\right) \backslash$ Int $B^{q} \subset P^{\prime} \cap U$ induces an isomorphism of the homotopy groups in dimensions $\leq \operatorname{dim}\left(P^{\prime} \cap U\right)-(l-1)(=$ in co-dimensions $\geq l-1)$. Also note that since $F \cap \partial B^{q}=\emptyset$, we have $F \cap \partial B^{m}=\left(F \cap \operatorname{Int} B^{q}\right) \times \partial B^{m-q}$ and therefore $\operatorname{dim} F \cap \partial B^{m} \leq \operatorname{dim} F-1$.

Let $B_{*}^{m-q}$ be a cube lying in the geometric interior of $B^{m-q}$ such that the centers of $B_{*}^{m-q}$ and $B^{m-q}$ coincide and $\partial B^{q} \times B_{*}^{m-q} \subset \operatorname{Int}\left(\left(P_{0} \cup \cdots \cup P_{t}\right) \cap U\right)$.

Use the notations of 4.1. Define $F_{L}=(N \cap F) \cup S$, where $S=\partial B^{m-q}$. Consider $B^{q}$ as the join $O * \partial B^{q}$, where $O$ is the center of $B^{q}$, and define a decomposition $\mathcal{P}_{L}$ of $L$ as the collection of the sets $\mathcal{P}_{L}=\left\{P_{L}^{\prime}: P^{\prime} \in \mathcal{P}\right\}$, where $P_{L}^{\prime}=P^{\prime} \cap N$ if $P^{\prime} \in \mathcal{P}$ and $P^{\prime} \neq P_{0}, \ldots, P_{t}$ and $P_{L}^{\prime}=\left(P^{\prime} \cap N\right) \cup$ $\left(\left(O *\left(P^{\prime} \cap \partial B^{q}\right)\right) \times B_{*}^{m-q}\right)$ for $P^{\prime}=P_{i}, 0 \leq i \leq t$. Note that $\mathcal{P}_{L}$ restricted to $N$ coincides with $\mathcal{P}$ restricted to $N$. It is easy to see that $\mathcal{P}_{L}$ restricted to $B^{q} \times B_{*}^{m-q}$, to $\partial B^{q} \times\left(B^{m-q} \backslash \operatorname{Int} B_{*}^{m-q}\right)$, to $\partial B^{q} \times B_{*}^{m-q}$, to $B^{q} \times \partial B_{*}^{m-q}$ and to $\partial B^{q} \times \partial B_{*}^{m-q}$ are partitions. Then, by $2.2, \mathcal{P}_{L}$ is a partition on $L \backslash(N \cap F)$ and $\partial T \backslash(N \cap F)$ and hence $\mathcal{P}_{L}$ is a partition on $L \backslash F_{L}$ and $\partial T \backslash F_{L}$. For a finite intersection $P^{\prime}=P_{0}^{\prime} \cap \cdots \cap P_{j}^{\prime}$ of $P_{0}^{\prime}, \ldots, P_{j}^{\prime} \in \mathcal{P}$ such that $P^{\prime} \cap U \neq \emptyset$ define $P_{L}^{\prime}=\left(P_{0}^{\prime}\right)_{L} \cap \cdots \cap\left(P_{j}^{\prime}\right)_{L}$.

Now apply the black hole modification to $\mathcal{P}_{L}$ and $F_{L}$ with $S$ as the black hole of the modification. Note that since $S$ lies in $\partial N$, by 2.2 , for every finite intersection $P^{\prime}$ of $\mathcal{P}, P^{\prime} \cap U \cap N \cap S \subset \partial\left(P^{\prime} \cap U \cap N\right)$ and hence the inclusion $\left(P^{\prime} \cap U \cap N\right) \backslash S \subset P^{\prime} \cap U \cap N$ induces an isomorphism of the 
homotopy groups in all dimensions. Recall that $f\left(S_{P}\right) \subset P \cap U \cap N$. By 4.1 and the construction of $\mathcal{P}_{L}$ one can easily verify that for every finite intersection $P^{\prime}$ of $\mathcal{P}$ such that $P^{\prime} \cap U \neq \emptyset$ and $P \neq P^{\prime},\left(P^{\prime} \cap U \cap N\right) \backslash S$ is a deformation retract of $\left(P_{L}^{\prime}\right)^{\pi} \backslash F_{L}^{\pi}$ and, for the intersection $P$, the inclusion of $(P \cap U \cap N) \backslash S$ into $P_{L}^{\pi} \backslash F_{L}^{\pi}$ induces an isomorphism of the homotopy groups in dimensions $<q-t-1$ and an epimorphism in dimension $q-t-1$ such that the element of the homotopy group represented by the map $f$ is in the kernel of this epimorphism.

Thus for every intersection $P^{\prime}$ of $\mathcal{P}$ such that $P^{\prime} \cap U \neq \emptyset$ the connectivity of $\left(P_{L}^{\prime}\right)^{\pi} \backslash F_{L}^{\pi}$ fits the connectivity of $P^{\prime} \backslash F$ for $P^{\prime} \neq P$ and we contributed toward improving the connectivity of $P \backslash F$ in its modification $P_{L}^{\pi} \backslash F_{L}^{\pi}$.

Define $F^{\pi}=F_{L}^{\pi},\left(P^{\prime}\right)^{\pi}=\left(P_{L}^{\prime}\right)^{\pi}$ for a finite intersection $P^{\prime}$ of $\mathcal{P}$ and let $\mathcal{P}^{\pi}=\left\{\left(P^{\prime}\right)^{\pi}: P^{\prime} \in \mathcal{P}\right\}=\mathcal{P}_{L}^{\pi}$. It is easy to see that the one-to-one correspondence between $\mathcal{P}$ and $\mathcal{P}_{L}$ defined by $P^{\prime} \mapsto\left(P^{\prime}\right)_{L}, P^{\prime} \in \mathcal{P}$, becomes a matching of partitions when $\mathcal{P}$ and $\mathcal{P}_{L}$ are restricted to $M \backslash F$ and $L \backslash F_{L}$ respectively. Then by 4.1 the one-to-one correspondence between $\mathcal{P}$ and $\mathcal{P}^{\pi}$ defined by $P^{\prime} \rightarrow\left(P^{\prime}\right)^{\pi}, P^{\prime} \in \mathcal{P}$, becomes a matching of partitions when $\mathcal{P}$ and $\mathcal{P}^{\pi}$ are restricted to $M \backslash F$ and $M \backslash F^{\pi}$ respectively. Note that $F^{\pi} \subset \operatorname{Int} M$ and because $\operatorname{dim} F \cap \partial B^{m} \leq \operatorname{dim} F-1$ we have $\operatorname{dim} F^{\pi} \leq$ $\max \{\operatorname{dim} S, \operatorname{dim} F\}=\max \{m-q-1, \operatorname{dim} F\}$.

Finally, note that even if $F=\emptyset$ then $F^{\pi}=S$ and we improve the connectivity of $P$ at the expense of an "irregular" behavior of $\mathcal{P}^{\pi}$ on the black hole $S$, in particular, we create additional intersections of $\mathcal{P}^{\pi}$ on $S$ and the elements of $\mathcal{P}^{\pi}$ are no longer manifolds in $M$.

4.3. A discretization of images of maps. Assume that $M$ is a triangulated manifold such that $\operatorname{dim} M \geq 2 r+1$ and $\mathcal{U}$ is an open cover of $M$.

Let $\mathcal{F}$ be countable collection of maps $f: S^{r} \rightarrow M$ from a triangulated $r$-dimensional sphere $S^{r}$. Then there is a PL-subcomplex $R$ of $M$ of dimension $\leq r$ lying in Int $M$ such that every map $f$ in $\mathcal{F}$ admits a $\mathcal{U}$-close approximation $f^{\prime}: S^{r} \rightarrow \operatorname{Int} M \backslash R$ such that $f^{\prime}$ is a PL-embedding and the images of the maps in $\mathcal{F}^{\prime}=\left\{f^{\prime}: f \in \mathcal{F}\right\}$ form a discrete family in $M \backslash R$. Moreover, given a decomposition $\mathcal{P}$ of $M$, the set $R$ can be chosen so that $R$ is nowhere dense on the finite intersections of $\mathcal{P}$, that is, the intersection of $R$ with every finite intersection of $\mathcal{P}$ is nowhere dense in that intersection.

Indeed, arrange $\mathcal{F}$ into a sequence $\mathcal{F}=\left\{f_{1}, f_{2}, \ldots\right\}$ and take a sufficiently small triangulation $\mathcal{T}$ of $M$ such that $\mathcal{T}$ underlies $\mathcal{P}$. Approximate each $f_{i}: S^{r} \rightarrow M$ by a map $f_{i}^{\prime \prime}: S^{r} \rightarrow M$ such that $f_{i}^{\prime \prime}\left(S^{r}\right)$ is contained in $R^{\prime}=$ the $r$-skeleton of $\mathcal{T}$. Fix a metric $d$ on $M$ and approximate each $f_{i}^{\prime \prime}$ by a PL-embedding $f_{i}^{\prime \prime \prime}: S^{r} \rightarrow M$ such that $f_{i}^{\prime \prime \prime}\left(S^{r}\right) \cap R^{\prime}=\emptyset, d\left(f_{i}^{\prime \prime \prime}(x), R^{\prime}\right) \leq 1 / i$ for every $x \in S^{r}$ and $f_{i}^{\prime \prime \prime}\left(S^{r}\right) \cap f_{j}^{\prime \prime \prime}\left(S^{r}\right)=\emptyset$ for $i \neq j$. Then the sets $f_{i}^{\prime \prime \prime}\left(S^{r}\right)$, $i=1,2, \ldots$, form a discrete family in $M \backslash R^{\prime}$. Now take a PL-embedding 
$g: M \rightarrow M$ such that $g(M)$ is a PL-subcomplex of $M, g(M) \subset$ Int $M$, the restriction of $g$ to $R^{\prime}$ is in general position with $\mathcal{T}$, and $g$ is close to the identity map of $M$. It is clear that the construction can be carried out so that $f_{i}^{\prime}=g \circ f_{i}^{\prime \prime \prime}$ is $\mathcal{U}$-close to $f_{i}$. Then the maps $f_{i}^{\prime}$ and the set $R=g\left(R^{\prime}\right)$ will have the required properties.

In a similar way one can show that if $\mathcal{F}$ is a countable collection of maps $f: B^{r} \rightarrow M$ from a triangulated $r$-dimensional ball $B^{r}$ such that, for every $f$ in $\mathcal{F}, f$ restricted to $\partial B^{r}$ is a PL-embedding and the images of the maps in $\left\{\left.f\right|_{\partial B^{r}}: f \in \mathcal{F}\right\}$ form a discrete family in $M$, then there is a PL-subcomplex $R$ of $M$ of dimension $\leq r$ lying in $\operatorname{Int} M$ such that every map $f$ in $\mathcal{F}$ admits a $\mathcal{U}$-close approximation $f^{\prime}: B^{r} \rightarrow M \backslash R$ such that $f^{\prime}$ is a PL-embedding, $\left.f^{\prime}\right|_{\partial B^{r}}=\left.f\right|_{\partial B^{r}}, f^{\prime}\left(\operatorname{Int} B^{r}\right) \subset \operatorname{Int} M$ and the images of the maps in $\mathcal{F}^{\prime}=\left\{f^{\prime}: f \in \mathcal{F}\right\}$ form a discrete family in $M \backslash R$. Moreover, for a given decomposition $\mathcal{P}$ of $M$, the set $R$ can be chosen so that $R$ is nowhere dense on the finite intersections of $\mathcal{P}$.

Note that if $M$ is $(r-1)$-connected and $R$ is a closed PL-presented subset of $M$ of dimension $\leq r$, then $M^{\prime}=M \backslash R$ is $(r-1)$-connected. In addition, if $\mathcal{P}$ is a decomposition of $M$ which is an $l$-co-connected partition on an open subset $U$ of $M$ with $l \geq r+2$ and $R$ is nowhere dense on the finite intersections of $\mathcal{P}$, then $\mathcal{P}^{\prime}=\{P \backslash R: P \in \mathcal{P}\}$ is a decomposition of $M^{\prime}$ which is an l-co-connected partition on $U^{\prime}=U \backslash R$ and the one-to-one correspondence between $\mathcal{P}$ and $\mathcal{P}^{\prime}$ defined by $P \mapsto P \backslash R, P \in \mathcal{P}$, induces a matching of partitions when $\mathcal{P}$ and $\mathcal{P}^{\prime}$ are restricted to $U$ and $U^{\prime}$ respectively (the property that $R$ is nowhere dense on the finite intersections of $\mathcal{P}$ is needed to guarantee that for every finite intersection $P$ of $\mathcal{P},(P \cap U) \backslash R \neq \emptyset$ provided $P \cap U \neq \emptyset)$.

4.4. Improving connectivity of intersections simultaneously. Adopt the notations and the assumptions of 4.2. We are going to show how to improve simultaneously the connectivity of all the intersections of $\mathcal{P} \mid U$ of dimension $m-t$ to $(l-1)$-co-connectivity.

For an intersection $P$ of $\mathcal{P}$ with $\operatorname{dim} P \cap U=m-t$ choose a countable collection $\mathcal{F}_{P}$ of PL-embeddings $f: S_{P} \rightarrow \operatorname{Int}(P \cap U)$ from a $(q-t-1)$ dimensional sphere $S_{P}$ that generate the $(q-t-1)$-dimensional homotopy group of $P \cap U$. Since $F$ is a PL-subcomplex of $M$ we may assume that the images of the maps in $\mathcal{F}_{P}$ lie outside a neighborhood of $F$. Then by 4.3 one can find a PL-subcomplex $R_{P}$ of $M$ such that $R_{P} \subset \operatorname{Int}(P \cap U)$ and $\operatorname{dim} R_{P} \leq q-t-1$ and assume that the images of the embeddings in $\mathcal{F}_{P}$ are contained in $\operatorname{Int}\left((P \cap U) \backslash R_{P}\right)$ and form a discrete family in $M \backslash R_{P}$.

Recall that in 4.2 the elements of $\mathcal{P}$ that form the intersection $P$ are enumerated according to the vertices of a sample simplex $\Delta=\left\{v_{0}, \ldots, v_{t}\right\}$ (we fix such an enumeration arbitrarily and independently for every intersection 
$P$ of $\mathcal{P}$ with $\operatorname{dim} P \cap U=m-t$ ). Following 4.2 and using 4.3 we can define for every $\Delta^{\prime} \subset \Delta$ a set $R_{P}^{\Delta^{\prime}}$ and collections of maps $\mathcal{F}_{P}^{\Delta^{\prime}}=\left\{f_{\Delta^{\prime}}: f \in \mathcal{F}_{P}\right\}$ and $\mathcal{F}_{P}^{\partial \Delta^{\prime}}=\left\{f_{\partial \Delta^{\prime}}: f \in \mathcal{F}_{P}\right\}$ such that $R_{P}^{\Delta^{\prime \prime}} \subset R_{P}^{\Delta^{\prime}}$ if $\Delta^{\prime \prime} \subset \Delta^{\prime}, R_{P}^{\Delta^{\prime}}$ is a closed subset of $M$ lying in $P\left(\Delta^{\prime}\right) \cap U$, the images of the maps of $\mathcal{F}_{P}^{\Delta^{\prime}}$ are contained in $G_{P}^{\Delta^{\prime}}=\left(P\left(\Delta^{\prime}\right) \cap U\right) \backslash R_{P}^{\Delta^{\prime}}$ and form a discrete family in $M \backslash R_{P}^{\Delta^{\prime}}$, and $R_{P}^{\Delta^{\prime}} \backslash R_{P}^{\partial \Delta^{\prime}}$ is a PL-subcomplex of $M \backslash R_{P}^{\partial \Delta^{\prime}}$ lying in $\left(P\left(\Delta^{\prime}\right) \cap U\right) \backslash R_{P}^{\partial \Delta^{\prime}}$ such that $\operatorname{dim} R_{P}^{\Delta^{\prime}} \backslash R_{P}^{\partial \Delta^{\prime}} \leq q-t+\operatorname{dim} \Delta^{\prime}$ where $R_{P}^{\partial \Delta^{\prime}}=\bigcup\left\{R_{P}^{\Delta^{\prime \prime}}: \Delta^{\prime \prime} \subset \partial \Delta^{\prime}\right\}$. Here we assume that $\Delta^{\prime}=\emptyset$ is a simplex of $\Delta$ and $\mathcal{F}_{P}^{\emptyset}=\mathcal{F}_{P}$ and $R_{P}^{\emptyset}=R_{P}$, and we also assume that $P(\Delta)=M$.

Denote by $\mathcal{F}^{\partial \Delta}$ and $R^{\partial \Delta}$ the union of $\mathcal{F}_{P}^{\partial \Delta}$ and $R_{P}^{\partial \Delta}$ respectively over all the intersections $P$ of $\mathcal{P}$ with $\operatorname{dim} P \cap U=m-t$. In order to carry out the construction described above so that $R^{\partial \Delta}$ will be a closed PL-presented subset of $M$ and the images of the maps in $\mathcal{F}^{\partial \Delta}$ will form a discrete family in $M \backslash R^{\partial \Delta}$, we need to take into account that the same intersection $P^{\prime}$ of $\mathcal{P}$ with $\operatorname{dim} P^{\prime} \cap U>m-t$ can be involved in many (even countably many) intersections of dimension $m-t$. This can be done as follows. For a finite intersection $P^{\prime}$ of $\mathcal{P}$ with $\operatorname{dim} P^{\prime} \cap U>m-t$ denote by $R_{\partial P^{\prime}}, R_{P^{\prime}}$, $\mathcal{F}_{\partial P^{\prime}}$ and $\mathcal{F}_{P^{\prime}}$ the union of $R_{P}^{\partial \Delta^{\prime}}, R_{P}^{\Delta^{\prime}}, \mathcal{F}_{P}^{\partial \Delta^{\prime}}$ and $\mathcal{F}_{P}^{\Delta^{\prime}}$ respectively over all finite intersections $P$ of $\mathcal{P}$ such that $\operatorname{dim} P \cap U=m-t$ and all $\Delta^{\prime} \subset \partial \Delta$ such that $P\left(\Delta^{\prime}\right)=P^{\prime}$, and let $G_{P^{\prime}}=\left(P^{\prime} \cap U\right) \backslash R_{P^{\prime}}$. It is obvious that if $\operatorname{dim} P^{\prime} \cap U=m-t+1$ then $R_{\partial P^{\prime}}$ is closed in $M$, the images of the maps in $\mathcal{F}_{\partial P^{\prime}}$ are contained in $\partial\left(P^{\prime} \cap U\right) \backslash R_{\partial P^{\prime}}$ and form a discrete family in $M \backslash R_{\partial P^{\prime}}$. Now assume that the last properties hold for a finite intersection $P^{\prime}$ of $\mathcal{P}$ with $\operatorname{dim} P^{\prime} \cap U>m-t$. Then applying 4.3 to all the maps of $\mathcal{F}_{\partial P^{\prime}}$ we can enlarge $R_{\partial P^{\prime}}$ to a closed subset $R_{P^{\prime}}$ of $M$ contained in $P^{\prime} \cap U$ and find extensions $\mathcal{F}_{P^{\prime}}$ of the maps in $\mathcal{F}_{\partial P^{\prime}}$ such that $\operatorname{dim} R_{P^{\prime}} \leq \operatorname{dim}\left(P^{\prime} \cap U\right)-l+1=$ $\operatorname{dim}\left(P^{\prime} \cap U\right)+q-m-1, R_{P^{\prime}} \backslash R_{\partial P^{\prime}}$ is a PL-subcomplex of $M \backslash R_{\partial P^{\prime}}$, the images of the extensions $\mathcal{F}_{P^{\prime}}$ of the maps in $\mathcal{F}_{\partial P^{\prime}}$ are contained in $G_{P^{\prime}}$ and form a discrete family in $M \backslash R_{P^{\prime}}$. Thus we can define $R_{P}^{\Delta^{\prime}}=R_{P^{\prime}}$ for every finite intersection $P$ of $\mathcal{P}$ with $\operatorname{dim} P \cap U=m-t$ and $\Delta^{\prime} \subset \partial \Delta$ such that $P\left(\Delta^{\prime}\right)=P^{\prime}$. Then proceeding by induction on $\operatorname{dim} P^{\prime} \cap U$ we construct for every intersection $P$ of $\mathcal{P}$ with $\operatorname{dim} P \cap U=m-t$ and $\Delta^{\prime} \subset \partial \Delta$ the collection of maps $\mathcal{F}_{P}^{\Delta^{\prime}}$ and the set $R_{P}^{\Delta^{\prime}}$ such that $R^{\partial \Delta}$ is a closed PL-presented subset of $M$ contained in $U$ such that $\operatorname{dim} R^{\partial \Delta} \leq q-1$ and the images of the maps in $\mathcal{F}^{\partial \Delta}$ are contained in $(M \cap U) \backslash R^{\partial \Delta}$ and form a discrete family in $M \backslash R^{\partial \Delta}$. From the construction it is clear that $R^{\partial \Delta}$ is nowhere dense on the finite intersections of $\mathcal{P}$.

By 4.3 the set $R^{\partial \Delta}$ can be enlarged to a closed PL-presented subset $R$ of $M$ and each map $f_{\partial \Delta}: S^{q-1} \rightarrow M \backslash R^{\partial \Delta}$ in $\mathcal{F}^{\partial \Delta}$ can be extended to a map $f_{\Delta}^{\prime}: B^{q} \rightarrow M \backslash R$ such that $\operatorname{dim} R \leq q, R$ is nowhere dense on the finite intersections of $\mathcal{P}$ and the images of the maps $f_{\Delta}^{\prime}$ form a discrete family 
in $M \backslash R$. Hence for every $f_{\partial \Delta} \in \mathcal{F}^{\partial \Delta}$ there is an open subset $Q\left(f_{\partial \Delta}\right)$ of $M \backslash R$ containing the image of $f_{\partial \Delta}$ such that $\mathcal{Q}=\left\{Q\left(f_{\partial \Delta}\right): f_{\partial \Delta} \in \mathcal{F}^{\partial \Delta}\right\}$ is a discrete family in $M \backslash R$ and $f_{\partial \Delta}$ is null-homotopic in $Q\left(f_{\partial \Delta}\right)$. Then, by 5.2 below, the black hole modification used in 4.2 and involving $f_{\partial \Delta}$ can be carried out inside $Q\left(f_{\partial \Delta}\right)$.

Note that since $R$ is a PL-presented set of dimension $\leq q$ and $m \geq 2 q+1$, for every finite intersection $P$ of $\mathcal{P}$ that intersects $U$ we find that the inclusion $(P \cap U) \backslash R \subset P \cap U$ induces an isomorphism of the homotopy groups in dimensions $\leq \operatorname{dim} P \cap U-(l-1)(=$ co-dimensions $\geq l-1)$.

Thus, after removing $R$ from $M, F$ and the elements of $\mathcal{P}$, the black hole modification 4.1 used in 4.2 can be applied independently for every map in $\mathcal{F}^{\partial \Delta}$ in order to modify $\mathcal{P}$ to a decomposition $\mathcal{P}^{\prime}$ of $M^{\prime}=M \backslash R$ and $F$ to a set $F^{\prime}$ such that $\mathcal{P}^{\prime}=$ the result of all the modifications $\mathcal{P}^{\pi}$ and $F^{\prime}=$ the result of all the modifications $F^{\pi}$. Then it is easy to derive from 4.2 that $F^{\prime}$ is a PL-subcomplex of $M^{\prime}$ of dimension $\leq \max \{m-q-1, \operatorname{dim} F\}$ lying in Int $M^{\prime}, \mathcal{P}^{\prime}$ is a partition on $M^{\prime} \backslash F^{\prime}$ and for every intersection $P$ of $\mathcal{P}$ with $P \cap U \neq \emptyset, P$ is modified to $P^{\prime}$ (= the result of all the modifications $P^{\pi}$ ) such that $P^{\prime} \cap\left(M^{\prime} \backslash F^{\prime}\right)$ is $l$-co-connected and $P^{\prime} \cap\left(M^{\prime} \backslash F^{\prime}\right)$ is $(l-1)$-co-connected if $\operatorname{dim} P \cap U \geq m-t$.

Note that the natural one-to-one correspondence between $\mathcal{P}$ and $\mathcal{P}^{\prime}$ defined by sending $P \in \mathcal{P}$ to its modification $P^{\prime} \in \mathcal{P}^{\prime}$ turns into a matching of partitions when $\mathcal{P}$ and $\mathcal{P}^{\prime}$ are restricted to $M \backslash F$ and $M^{\prime} \backslash F^{\prime}$.

It is clear that the images of the maps in $\mathcal{F}^{\partial \Delta}$ are contained in the elements of st $\mathcal{P}$. Assume that $\mathcal{W}$ is an open cover of $M$ such that for every $P \in \mathcal{P}$ there is $W \in \mathcal{W}$ such that $\operatorname{st}(P, \mathcal{P}) \subset W$ and the inclusion of st $(P, \mathcal{P})$ into $W$ induces the zero-homomorphism of the homotopy groups in dimensions $\leq q-1$. By 4.3 the collection $\mathcal{Q}$ can be chosen so that for every $f_{\partial \Delta} \in \mathcal{F}^{\partial \Delta}, Q\left(f_{\partial \Delta}\right) \subset \operatorname{st}\left(f_{\partial \Delta}\left(S^{q-1}\right)\right.$, st $\left.\mathcal{W}\right)$.

Then it is easy to see for every $P^{\prime} \in \mathcal{P}^{\prime}$ that $P^{\prime} \subset \operatorname{st}\left(P, \mathrm{st}^{2} \mathcal{W}\right)$, where $P^{\prime}$ is the modification of $P \in \mathcal{P}$.

4.5. Absorbing simplexes. Let $M$ be a triangulated $(q-1)$-connected $m$-dimensional manifold with $m \geq 2 q+1$ and let $l=m-q+1$. Assume that $F$ is a PL-subcomplex of $M$ lying in Int $M$ such that $U=M \backslash F$ is $l$-co-connected and $\operatorname{dim} F \leq m-q$, and assume that $\mathcal{P}$ is a decomposition of $M$ such that $\mathcal{P}$ is an $l$-co-connected partition on $U$. Fix a triangulation $\mathcal{T}$ of $M$ for which $F$ is a subcomplex and let $F^{\prime}$ be the $(m-q-1)$-skeleton of $F$. We will show how to modify $M$ to an open subset $M^{\prime} \subset M$ and $\mathcal{P}$ to a decomposition $\mathcal{P}^{\prime}$ of $M^{\prime}$ such that $M \backslash M^{\prime}$ is a PL-subcomplex of $M$ of dimension $\leq q, \mathcal{P}^{\prime}$ restricted to $U^{\prime}=M^{\prime} \backslash F^{\prime}$ is an $l$-co-connected partition and $\mathcal{P}$ admits a natural one-to-one correspondence to $\mathcal{P}^{\prime}$ which becomes a matching of partitions when $\mathcal{P}$ and $\mathcal{P}^{\prime}$ are restricted to $U$ and $U^{\prime}$ respectively. 
Assume that the triangulation $\mathcal{T}$ that we fixed in $M$ is the second barycentric subdivision of a triangulation for which $F$ and the elements of $\mathcal{P}$ are subcomplexes. Let $\mathcal{T}_{F}$ be the collection of all $(m-q)$-dimensional simplexes lying in $F$ and for $\Delta \in \mathcal{T}_{F}$ let $S_{\Delta}$ and $\Delta * S_{\Delta}$ be the link of $\Delta$ and the join of $\Delta$ with $S_{\Delta}$ respectively which are defined as follows:

$$
\begin{aligned}
S_{\Delta} & =\bigcup\left\{\Delta^{\prime} \in \mathcal{T}: \Delta^{\prime} \cap \Delta=\emptyset \text { and there is } \Delta^{\prime \prime} \in \mathcal{T} \text { with } \Delta, \Delta^{\prime} \subset \Delta^{\prime \prime}\right\}, \\
\Delta * S_{\Delta} & =\bigcup\left\{\Delta^{\prime} \in \mathcal{T}: \Delta \subset \Delta^{\prime}\right\} .
\end{aligned}
$$

Let $Q$ be the union of $\operatorname{Int}\left(\Delta * S_{\Delta}\right)$ for all $\Delta$ in $\mathcal{T}_{F}$, and $N=M \backslash(Q \cup F)$. Note that for every $\Delta \in \mathcal{T}_{F}$ the link $S_{\Delta}$ of $\Delta$ is a $(q-1)$-dimensional sphere lying in Int $M$, and for different simplexes $\Delta_{1}$ and $\Delta_{2}$ in $\mathcal{T}_{F}$ the joins $\Delta_{1} * S_{\Delta_{1}}$ and $\Delta_{2} * S_{\Delta_{2}}$ do not intersect on $U$. Also note that for every subset $H$ which is a union of a finite intersection of $\mathcal{P}$ such that $H \cap U \neq \emptyset$ and for every $\Delta \in \mathcal{T}_{F}$ we find that $(H \cap U) \backslash \operatorname{Int}\left(\Delta * S_{\Delta}\right)$ is a deformation retract of $H \cap U$ and hence $H \cap N$ is a deformation retract of $H \cap U$. Then $N$ is an $l$-co-connected manifold (since $N$ is a deformation retract of $U$ ) and $\mathcal{P} \mid N$ is an $l$-co-connected partition (since $\mathcal{P} \mid U$ is $l$-co-connected).

Fix $\Delta \in \mathcal{T}_{F}$ and let an open subset $V_{\Delta}$ of $N$ be such that $S^{q-1}=S_{\Delta} \subset V_{\Delta}$ and the inclusion of $S^{q-1}$ into $V_{\Delta}$ is null-homotopic in $V_{\Delta}$. Then by 5.3 there are an element $P_{\Delta} \in \mathcal{P}$ and a PL-embedding of a cube $B^{m}=B^{q} \times B^{m-q}$ into Int $M$ such that $B^{m} \subset\left(\Delta * S_{\Delta}\right) \cup V_{\Delta}, \Delta=O \times B^{m-q}=F \cap B^{m}$, $P \cap U \cap B^{m}=\left(P \cap U \cap B^{q}\right) \times B^{m-q}$ for every $P \in \mathcal{P}, \partial B^{q} \times B^{m-q} \subset$ $\operatorname{Int}\left(P_{\Delta} \cap U\right), \mathcal{P}$ restricted to $\left(M \backslash\right.$ Int $\left.B^{m}\right) \cap U$ and to $\partial B^{m} \cap U$ are partitions and $\left(P \backslash \operatorname{Int} B^{m}\right) \cap U$ is a deformation retract of $\left(P \backslash \operatorname{Int} B^{q}\right) \cap U$ for every finite intersection $P$ of $\mathcal{P}$.

Let us briefly describe the general idea of the construction in 5.3. The inclusion of $S^{q-1}$ can be extended to a PL-embedding of a $q$-dimensional cube $B_{\#}^{q}$ into $V_{\Delta}$ such that $S^{q-1}=\partial B_{\#}^{q}$ and the embedding of $B_{\#}^{q}$ extends to a PL-embedding of an $m$-dimensional cube $B_{\#}^{m}=B_{\#}^{q} \times B_{\#}^{m-q}$ into $V_{\Delta}$ such that $\partial B_{\#}^{q} \times B_{\#}^{m-q} \subset B_{\#}^{m} \cap \partial\left(\Delta * S_{\Delta}\right)$ and $P \cap B_{\#}^{m}=\left(P \cap B_{\#}^{q}\right) \times B_{\#}^{m-q}$ for every $P \in \mathcal{P}$. Let $P_{\Delta} \in \mathcal{P}$ be such that $\operatorname{Int}\left(P_{\Delta} \cap B_{\#}^{q}\right) \neq \emptyset$ and let a $q$-dimensional cube $\sigma_{\#}$ be PL-embedded in $\operatorname{Int}\left(P_{\Delta} \cap B_{\#}^{q}\right)$. Then the cube $B^{m}=\left(\Delta * S_{\Delta}\right) \cup\left(\left(B_{\#}^{q} \backslash \operatorname{Int} \sigma_{\#}\right) \times B_{\#}^{m-q}\right)$ can be represented as the product $B^{m}=B^{q} \times B^{m-q}$ with the required properties. See 5.3 for details.

Let $B_{*}^{m-q}$ be a cube lying in the geometric interior of $B^{m-q}$. Use the notations of 4.1. Define $F_{L}=F \backslash$ Int $B^{m}, P_{L}=P \backslash$ Int $B^{m}$ if $P \neq P_{\Delta}$ and $P \in \mathcal{P},\left(P_{\Delta}\right)_{L}=\left(P_{\Delta} \backslash \operatorname{Int} B^{m}\right) \cup\left(B^{q} \times B_{*}^{m-q}\right)$ and define $\mathcal{P}_{L}=\left\{P_{L}: P \in \mathcal{P}\right\}$. Then $S=\partial B^{m-q}=\partial \Delta \subset F_{L}$ and it is easy to see that $\mathcal{P}_{L}$ is an $l$-coconnected partition on $L \backslash F_{L}$ and $\mathcal{P}_{L}$ is a partition on $\partial T \backslash F_{L}$. Now apply the black hole modification with the black hole $S=\partial \Delta$ and write $F^{\pi}=F_{L}^{\pi}$, 
$P^{\pi}=P_{L}^{\pi}$ for $P \in \mathcal{P}$ and $\mathcal{P}^{\pi}=\left\{P^{\pi}: P \in \mathcal{P}\right\}=\mathcal{P}_{L}^{\pi}$. Note that $F_{L}^{\pi}=F_{L}$ since $F \cap \partial B^{m}=\partial \Delta$. Then $\mathcal{P}^{\pi}$ is a decomposition of $M$ which is an $l$-coconnected partition on the manifold $M \backslash F^{\pi}=(M \backslash F) \cup \operatorname{Int} \Delta$ in which we have absorbed the geometric interior of $\Delta$ in the modification of $P_{\Delta}$. Note that the one-to-one correspondence between $\mathcal{P}$ and $\mathcal{P}_{L}$ defined by $P \mapsto P_{L}$, $P \in \mathcal{P}$, becomes a matching of partitions when $\mathcal{P}$ and $\mathcal{P}_{L}$ are restricted to $M \backslash F$ and $L \backslash F_{L}$ respectively. Then by 4.1 the one-to-one correspondence between $\mathcal{P}$ and $\mathcal{P}^{\pi}$ defined by $P \rightarrow P^{\pi}, P \in \mathcal{P}$, becomes a matching of partitions when $\mathcal{P}$ and $\mathcal{P}^{\pi}$ are restricted to $M \backslash F$ and $M \backslash F^{\pi}$ respectively.

Note that each $S_{\Delta}$ can be contracted to a point in $N$ outside some neighborhood of $F$ in $M$. Then by 4.3 there is a PL-subcomplex $R$ of $M$ contained in Int $N$ and nowhere dense on the finite intersections of $\mathcal{P}$ with $\operatorname{dim} R \leq q$ such that the collection $\mathcal{V}$ of the sets $V_{\Delta}, \Delta \in \mathcal{T}_{F}$, can be chosen to be discrete in $N \backslash R$. Then replacing $M$ by $M^{\prime}=M \backslash R$ and removing $R$ from the elements of $\mathcal{P}$ we can perform all the black hole modifications independently for all $\Delta \in \mathcal{T}_{F}$ and get from all the modifications $\mathcal{P}^{\pi}$ the corresponding decomposition $\mathcal{P}^{\prime}$ of $M^{\prime}$ with the required properties. It is clear that the natural one-to-one correspondence between $\mathcal{P}$ and $\mathcal{P}^{\prime}$ defined by sending each element of $\mathcal{P}$ to its modification becomes a matching of partitions when the decompositions are restricted to $M \backslash F$ and $M^{\prime} \backslash F^{\prime}$.

It is clear that the spheres $S_{\Delta}, \Delta \in \mathcal{T}_{F}$, are contained in the elements of st $\mathcal{P}$. Let $\mathcal{W}$ be an open cover of $M$ having the following property: for every $P \in \mathcal{P}$ there are $W \in \mathcal{W}$ and a set $H$ such that $\operatorname{st}(P, \mathcal{P}) \subset H \subset W, H$ is a union of elements of $\mathcal{P}$ and the inclusion st $(P, \mathcal{P}) \cap U \subset H \cap U$ induces the zero-homomorphism of the homotopy groups in dimensions $\leq q-1$. Then the inclusion $\operatorname{st}(P, \mathcal{P}) \cap N \subset H \cap N$ also induces the zero-homomorphism of the homotopy groups in dimensions $\leq q-1$ because $H \cap N$ is a deformation retract of $H \cap U$. Thus $S_{\Delta}$ can be contracted to a point in $\operatorname{st}(\Delta, \mathcal{W}) \cap N$. Then the collection $\mathcal{V}$ can be chosen so that for every $V_{\Delta} \in \mathcal{V}, V_{\Delta} \subset \operatorname{st}(\Delta$, st $\mathcal{W})$, and this implies that for every $P^{\prime} \in \mathcal{P}^{\prime}, P^{\prime} \subset \operatorname{st}\left(P, \mathrm{st}^{2} \mathcal{W}\right)$, where $P^{\prime}$ is the modification of $P \in \mathcal{P}$.

\section{Appendix}

5.1. Extending partitions in the black hole modification. This is a direct continuation of 4.1 and we adopt the notations and the assumptions of 4.1. This subsection is devoted to constructing the extensions $A^{\pi}, A \in \mathcal{A}$, the step that was omitted in 4.1 .

Recall that $T$ can be represented as the product $T=B^{q+1} \times S$, where $S$ is identified with $a \times S$ for a point $a$ in $\partial B^{q+1}$. We consider $B^{q+1}$ and $S$ as PL-embedded in Euclidean spaces. This induces the corresponding PLembedding of $T$ in the product of Euclidean spaces and we refer to these 
Euclidean spaces when properties of linearity are used; thus we say that a simplex is linear if it is a simplex (linearly spanned by its vertices) in the corresponding Euclidean space, and a map of a linear simplex is linear if it is the linear extension of its values on the vertices.

Denote by $p_{S}: T \rightarrow S$ and $p_{B}: T \rightarrow B^{q+1}$ the projections. Let $\mathcal{T}_{T}$ be a triangulation of $T$ such that the simplexes of $\mathcal{T}_{T}$ are linear, $p_{S}$ and $p_{B}$ restricted to every simplex of $\mathcal{T}_{T}$ are linear and $\mathcal{T}_{T}$ underlies $S$ and the simplexes of $\mathcal{T}$ contained in $T$. Let $\mathcal{T}_{T}^{\prime}$ be a subdivision of $\mathcal{T}_{T}$ such that the simplexes of $\mathcal{T}_{T}^{\prime}$ are linear and, for every simplex $\Delta$ of $\mathcal{T}_{T}, p_{S}(\Delta)$ is a subcomplex of $S$ with respect to $\mathcal{T}_{T}^{\prime}$.

Denote by $\mathcal{T}_{T}^{\prime \prime}$ the (first) barycentric subdivision of $\mathcal{T}_{T}^{\prime}$ and let $\mathcal{B}_{T}$ be the partition of $T$ formed by the stars of the vertices of $\mathcal{T}_{T}^{\prime}$ with respect to $\mathcal{T}_{T}^{\prime \prime}$. Then $\mathcal{B}_{S}=\mathcal{B}_{T} \mid S$ is a partition of $S$. For a non-empty finite intersection $B$ of $\mathcal{B}_{S}$ which is the intersection of distinct elements $B_{0}, \ldots, B_{t}$ of $\mathcal{B}_{S}$ let the vertex $v_{B}$ of $\mathcal{T}_{T}^{\prime \prime}$ be the barycenter of the simplex of $\mathcal{T}_{T}^{\prime}$ spanned by the vertices contained in $B_{0} \cup \cdots \cup B_{t}$.

For a finite intersection $B$ of $\mathcal{B}_{S}$ define $K_{B}=p_{S}^{-1}(B), N_{B}=p_{S}^{-1}(\partial B)$ and $M_{B}=K_{B} \cap \partial T$. Note that $K_{B}=B^{q+1} \times B, N_{B}=B^{q+1} \times \partial B$, $M_{B}=\partial B^{q+1} \times B, \partial K_{B}=N_{B} \cup M_{B}$ and $N_{B} \cap M_{B}=\partial B^{q+1} \cap \partial B$.

Let us show that $\mathcal{P}_{L}$ restricted to $M_{B} \backslash F$ and $\mathcal{P}_{L}$ restricted to $\partial M_{B} \backslash F$ are partitions. Assume that $B \in \mathcal{B}_{S}$ (that is, $\operatorname{dim} B=\operatorname{dim} S$ ). Note that $\mathcal{P}_{L}$ restricted to Int $M_{B} \backslash F_{L}$ is a partition. Take a point $x \in \partial M_{B}$ and let $\Delta_{x}$ be the smallest simplex of $\mathcal{T}_{T}$ containing $x$. Then $v_{B} \in p_{S}\left(\Delta_{x}\right)$ and let $y \in \Delta_{x}$ be such that $p_{S}(y)=v_{B}$. Let $G$ be a neighborhood of $x$ in $\partial M_{B}$ such that $G$ is contained in the star of $y$ with respect to $\mathcal{T}_{T}$. Then $(z, t) \mapsto z(1-t)+t y$, $z \in G, 0 \leq t<1$, defines a PL-embedding of $G \times[0,1)$ into $M_{B}$ such that $G \times[0,1)$ is a neighborhood of $x$ in $M_{B}$ and for every simplex $\Delta \in \mathcal{T}_{T}$ we have $\Delta \cap(G \times[0,1))=(G \cap \Delta) \times[0,1)$. Recall that $\mathcal{T}_{T}$ underlies $\mathcal{P}_{L} \mid \partial T$ and $F_{L} \cap \partial T$, and note that $G \times(0,1)$ is an open subset of $\partial T$. Therefore $\mathcal{P}_{L}$ restricted to $(G \times(0,1)) \backslash F_{L}$ is a partition. All these facts together imply by 2.2 that $\mathcal{P}_{L}$ restricted $(G \times[0,1)) \backslash F_{L}$ and $\mathcal{P}_{L}$ restricted to $G \backslash F_{L}$ are partitions and hence $\mathcal{P}_{L}$ restricted to $M_{B} \backslash F_{L}$ and $\mathcal{P}_{L}$ restricted to $\partial M_{B} \backslash F_{L}$ are partitions.

Now assume that for a finite intersection $B^{\prime} \in \mathcal{B}_{S}$ we already showed that $\mathcal{P}_{L}$ restricted to $M_{B^{\prime}} \backslash F_{L}$ and $\mathcal{P}_{L}$ restricted to $\partial M_{B^{\prime}} \backslash F_{L}$ are partitions, and let $B$ be a finite intersection of $\mathcal{B}_{S}$ such that $B \subset \partial B^{\prime}$ and $\operatorname{dim} B+1=\operatorname{dim} B^{\prime}$. Then replacing $S$ by $\partial B^{\prime}$ and $T$ by $B^{q+1} \times \partial B^{\prime}$ we can repeat the above reasoning to show that $\mathcal{P}_{L}$ restricted to $M_{B} \backslash F_{L}$ and $\mathcal{P}_{L}$ restricted to $\partial M_{B} \backslash F_{L}$ are partitions. Thus we have shown that for every finite intersection of $\mathcal{B}_{S}, \mathcal{P}_{L}$ restricted to $M_{B} \backslash F_{L}$ and to $\partial M_{B} \backslash F_{L}$ are partitions. 
Let $B$ be a non-empty finite intersection of $\mathcal{B}_{S}$. Denote by $V$ the set of vertices $v$ of $\mathcal{T}_{T}^{\prime \prime}$ such that $v \neq v_{B}, v \in \partial T$ and $\left[v, v_{B}\right]$ is a simplex of $\mathcal{T}_{T}^{\prime \prime}$. For each $v \in V$ choose a point $v^{\prime}$ lying on the interval $\left[v, v_{B}\right]$ connecting $v$ and $v_{B}$ as follows. If $v \in S$ then set $v^{\prime}=v$ and note that in this case $v^{\prime} \in \partial B$. If $v \notin S$ then choose $v^{\prime}$ such that $v^{\prime} \neq v_{B}, v^{\prime} \neq v$ and $p_{S}\left(\left[v^{\prime}, v\right]\right) \subset \operatorname{Int} B$. For a simplex $\Delta$ of $\mathcal{T}_{T}^{\prime \prime}$ spanned by vertices $v_{B}, v_{1}, \ldots, v_{k}$, with $v_{1}, \ldots, v_{k} \in V$ denote by $\Delta^{\prime}$ the simplex spanned by the vertices $v_{B}, v_{1}^{\prime}, \ldots, v_{k}^{\prime}$ and denote by $W_{B}$ the union of all such simplexes $\Delta^{\prime}$. Then $W_{B}$ is a PL-ball which is a closed neighborhood of $v_{B}$ in $M_{B}$ such that $W_{B} \cap S=M_{B} \cap S=B$ and for every $x \in \partial W_{B}$ the interval $\left[v_{B}, x\right]$ is contained in $W_{B}$ and $\left[v_{B}, x\right] \cap \partial W_{B}=x$. Let $\pi_{B}^{W}: W_{B} \backslash v_{B} \rightarrow \partial W_{B}$ be the radial projection. Using a PL-homeomorphism represent $K_{B}$ as $W_{B} \times[0,1]$ so that $W_{B}$ is identified with $W_{B} \times 0$ and extend $\pi_{B}^{W}$ to the radial projection

$\pi_{B}: K_{B} \backslash v_{B}=\left(W_{B} \times[0,1]\right) \backslash v_{B} \rightarrow \partial K_{B} \backslash \operatorname{Int} W_{B}=\left(\partial W_{B} \times[0,1]\right) \cup\left(W_{B} \times 1\right)$

( $\pi_{B}$ is defined with respect to the linear structure of $W_{B} \times[0,1]$ induced by the linear structures of $W_{B}$ and $\left.[0,1]\right)$.

Let us show that $\mathcal{P}_{L}$ restricted $M_{B} \backslash\left(F_{L} \cup \operatorname{Int} W_{B}\right)$ is a partition. Take $x \in \partial W_{B} \backslash S$. Then there are an open neighborhood $G$ of $x$ in $\partial W_{B} \backslash S$ and $\varepsilon>0$ such that $G \times[0, \varepsilon)$ is embedded into $M_{B} \backslash\left(S \cup \operatorname{Int} W_{B}\right)$ by the map $(z, t) \mapsto z(1+t)-t v_{B}, z \in G$, so that for every simplex $\Delta$ of $\mathcal{T}_{T}^{\prime \prime}$, $\Delta \cap(G \times[0, \varepsilon))=(\Delta \cap G) \times[0, \varepsilon)$. Recall that $\mathcal{T}_{T}^{\prime \prime}$ underlies $\mathcal{P}_{L}$, and $F_{L}$ restricted to $\partial T$ and $\mathcal{P}_{L}$ restricted to $M_{B} \backslash F_{L}$ and to $\partial M_{B} \backslash F_{L}$ are partitions. Since $G \times(0, \varepsilon)$ is an open subset of $M_{B}, \mathcal{P}_{L}$ restricted to $(G \times(0, \varepsilon)) \backslash F_{L}$ is a partition and therefore, by $2.2, \mathcal{P}_{L}$ restricted to $G \backslash F_{L}$ is a partition. Then, again by $2.2, \mathcal{P}_{L}$ restricted to $(G \times[0, \varepsilon)) \backslash F_{L}$ is a partition and hence $\mathcal{P}_{L}$ restricted $M_{B} \backslash\left(F_{L} \cup \operatorname{Int} W_{B}\right)$ is a partition (recall that $\left.S \subset F_{L}\right)$.

For every $A \in \mathcal{A}$ and every finite intersection $B$ of $\mathcal{B}_{S}$ define sets $A^{B}$ and $A^{\partial B}$ as follows. For $B=\emptyset$ set $A^{\emptyset}=A$ if $A \cap T=\emptyset$ and $A^{\emptyset}=A \cup S$ if $A \cap T \neq \emptyset$, and for $\operatorname{dim} B=0$ set $A^{\partial B}=A^{\emptyset}$. Now by induction on $\operatorname{dim} B$ define $A^{B}$ and $A^{\partial B}$ so that $A^{B}=A^{\partial B} \cup \pi_{B}^{-1}\left(A^{\partial B} \cap\left(\partial K_{B} \backslash \operatorname{Int} W_{B}\right)\right)$ for $\operatorname{dim} B \geq 0$ and $A^{\partial B}=\bigcup\left\{A^{B^{\prime}}: B^{\prime} \subset \partial B\right\}$ for $\operatorname{dim} B \geq 1$. Clearly, $A^{B}$ and $A^{\partial B}$ are PL-subcomplexes of $M$. Note that if $A \cap T=\emptyset$ then $A^{B}=A^{\partial B}=A$ for every $B$.

From the construction of $W_{B}$ and $\pi_{B}$ it follows that for every simplex $\Delta \in$ $\mathcal{T}_{T}^{\prime}$ intersecting $W_{B}$ we have $\pi_{B}^{-1}\left(\Delta \cap \partial W_{B}\right) \backslash S=\left(\Delta \cap W_{B}\right) \backslash S$. Then it is easy to see that $S^{\partial B}=S^{B}=S,\left(A^{\partial B} \cap M_{B}\right) \backslash S=\left(A^{B} \cap M_{B}\right) \backslash S=\left(A \cap M_{B}\right) \backslash S$ and hence $\left(A^{B_{1}} \cap A^{B_{2}}\right) \backslash S=A^{B_{1} \cap B_{2}} \backslash S,\left(A_{1}^{B} \cap A_{2}^{B}\right) \backslash S=\left(A_{1} \cap A_{2}\right)^{B} \backslash S$ for any $A, A_{1}, A_{2} \in \mathcal{A}$ and finite intersections $B, B_{1}, B_{2}$ of $\mathcal{B}_{S}$.

Define $\mathcal{P}^{B}=\left\{P^{B}: P \in \mathcal{P}_{L}\right\}$ and $\mathcal{P}^{\partial B}=\left\{P^{\partial B}: P \in \mathcal{P}_{L}\right\}$. Recall that $S \subset F_{L}$. Let us show by induction on $\operatorname{dim} B$ that $\mathcal{P}^{B}$ restricted to $K_{B} \backslash F_{L}^{B}$ and $\mathcal{P}^{\partial B}$ restricted to $N_{B} \backslash F_{L}^{\partial B}$ are partitions. 
For $\operatorname{dim} B=0$ we have $N_{B}=\emptyset$ and therefore $\mathcal{P}^{\partial B}$ restricted to $N_{B} \backslash F_{L}^{\partial B}$ is vacuously a partition. Let $B$ be a finite intersection of $\mathcal{B}_{S}$ such that $\mathcal{P}^{\partial B}$ restricted to $N_{B} \backslash F_{L}^{\partial B}$ is a partition. Define $R_{B}=\partial K_{B} \backslash \operatorname{Int} W_{B}$. From the construction of $\pi_{B}$ it follows that $K_{B} \backslash v_{B}$ is PL-homeomorphic to $R_{B} \times[0,1)$ so that $R_{B}$ corresponds to $R_{B} \times 0, \mathcal{P}^{B}$ restricted to $K_{B} \backslash v_{B}$ corresponds to the decomposition $\left(\mathcal{P}^{\partial B} \mid R_{B}\right) \times[0,1)$ of $R_{B} \times[0,1)$ and $\left(F_{L}^{B} \cap K_{B}\right) \backslash v_{B}$ corresponds to $\left(F_{L}^{\partial B} \cap R_{B}\right) \times[0,1)$. Note that $F_{L}^{\partial B} \cap M_{B}=F_{L} \cap M_{B}$ and $\mathcal{P}^{\partial B}$ restricted to $M_{B} \backslash S$ coincides with $\mathcal{P}_{L}$ restricted $M_{B} \backslash S$, and recall that $\mathcal{P}_{L}$ restricted to $\partial M_{B} \backslash F_{L}$ and to $M_{B} \backslash\left(F_{L} \cup \operatorname{Int} W_{B}\right)$ are partitions. Then, by $2.2, \mathcal{P}^{\partial B}$ restricted to $R_{B} \backslash F_{L}^{\partial B}$ is a partition and therefore $\mathcal{P}^{B}$ restricted to $K_{B} \backslash F_{L}^{B}$ is also a partition.

Now assume $B$ is a finite intersection of $\mathcal{B}_{S}$ such that $\operatorname{dim} B>0$ and for every finite intersection $B^{\prime}$ of $\mathcal{B}_{S}$ such that $B^{\prime} \subset \partial B$ we knew that $\mathcal{P}^{B^{\prime}}$ restricted to $K_{B^{\prime}} \backslash F_{L}^{B^{\prime}}$ is a partition. Then, by $2.2, \mathcal{P}^{\partial B}$ is a partition of $N_{B} \backslash F_{L}^{\partial B}$ since $\left\{K_{B^{\prime}}: B^{\prime}\right.$ is a finite intersection of $\mathcal{B}_{S}$ such that $\left.B^{\prime} \subset \partial B\right\}$ is a partition of $N_{B}$. The induction is complete.

Thus we have shown that $\mathcal{P}^{B}$ restricted to $K_{B} \backslash F_{L}^{B}$ is a partition for every finite intersection $B$ of $\mathcal{B}_{S}$. For $A \in \mathcal{A}$ write $A^{\pi}=\bigcup\left\{A^{B}: B \in \mathcal{B}_{S}\right\}$ and define $\mathcal{P}_{L}^{\pi}=\left\{P^{\pi}: P \in \mathcal{P}_{L}\right\}$. Note that $F_{L}^{B}=K_{B} \cap F_{L}^{\pi}$ and $\mathcal{P}_{L}^{\pi}$ restricted to $K_{B}$ coincides with $\mathcal{P}^{B}$. Then, by $2.2, \mathcal{P}_{L}^{\pi}$ is a partition on $T \backslash F_{L}^{\pi}$ since $\left\{K_{B}: B\right.$ is a finite intersection of $\left.\mathcal{B}_{S}\right\}$ is a partition of $T$. It is obvious that $\mathcal{P}_{L}^{\pi}$ restricted to $L \backslash S$ coincides with $\mathcal{P}_{L}$ restricted to $L \backslash S$ and therefore $\mathcal{P}_{L}^{\pi}$ is a decomposition of $M$ which is a partition on $M \backslash F_{L}^{\pi}$.

Now let us show that for $A_{1}, A_{2} \in \mathcal{A},\left(A_{1} \backslash A_{2}\right) \backslash S$ is a strong deformation retract of $\left(A_{1}^{\pi} \backslash A_{2}^{\pi}\right) \backslash S$. To this end it is enough to show that for every finite intersection $B$ of $\mathcal{B}_{S},\left(C_{1}^{\prime} \backslash C_{2}^{\prime}\right) \backslash S$ is a strong deformation retract of $\left(C_{1} \backslash C_{2}\right) \backslash S$ where $C_{i}^{\prime}=A_{i}^{\partial B} \cap \partial K_{B}$ and $C_{i}=A_{i}^{B} \cap K_{B}$. From the construction it follows that $\left(C_{1} \backslash C_{2}\right) \backslash S$ can be topologically represented as $C^{\prime} \times[0,1)$ with $C^{\prime}=\left(C_{1}^{\prime} \backslash C_{2}^{\prime}\right) \backslash\left(S \cup \operatorname{Int} W_{B}\right)$ so that $\left(C_{1}^{\prime} \backslash C_{2}^{\prime}\right) \backslash S$ is identified with $\left(C^{\prime} \times 0\right) \cup\left(\left(C^{\prime} \cap \partial W_{B}\right) \times[0,1)\right)$. Note that $C^{\prime}$ is a space admitting a triangulation for which $C^{\prime} \cap \partial W_{B}$ is a subcomplex of $C^{\prime}$ and therefore $\left(C^{\prime} \times 0\right) \cup\left(\left(C^{\prime} \cap \partial W_{B}\right) \times[0,1)\right)$ is a strong deformation retract of $C^{\prime} \times[0,1)$.

Let $\Delta \in \mathcal{T}_{T}$ be contained in $\partial T$. Since $p_{S}$ is linear on $\Delta$ and $p_{S}(\Delta)$ is a subcomplex of $\mathcal{T}_{T}^{\prime}$ we can conclude that $\operatorname{dim} \Delta \cap M_{B} \leq \operatorname{dim} \Delta+\operatorname{dim} B-\operatorname{dim} S$ for every finite intersection $B$ of $\mathcal{B}_{S}$. Then, as $\operatorname{dim} \Delta^{B} \backslash S \leq \operatorname{dim}\left(\Delta \cap M_{B}\right) \backslash$ $S+1$ for $\operatorname{dim} B=0$ and $\operatorname{dim} \Delta^{B} \backslash S \leq \operatorname{dim} \Delta^{\partial B} \backslash S+1$ for $\operatorname{dim} B>0$, we get $\operatorname{dim} \Delta^{\pi} \backslash S \leq \operatorname{dim} \Delta \backslash S+1$. Thus $\operatorname{dim}\left(A^{\pi} \cap T\right) \backslash S \leq \operatorname{dim}(A \cap \partial T) \backslash S+1$ for every $A \in \mathcal{A}$.

The remaining properties required in 4.1 are easy to verify. 


\subsection{Digging holes for improving connectivity of an intersection}

Summary. Here we present a construction used in 4.2. Let $M$ be a triangulated $m$-dimensional manifold, $F$ a PL-subcomplex of $M$ and $\mathcal{P}$ a decomposition of $M$ which is a partition on $M \backslash F$. Assume that $M$ is $(q-1)$-connected, $m \geq 2 q+1$ and let $S^{q-1} \subset$ Int $M \backslash F$ be a PL-embedded $q$-dimensional sphere so that $\mathcal{P}$ restricted to $S^{q-1}$ is a partition and for every finite intersection $P^{\prime}$ of $\mathcal{P}$ with $P^{\prime} \cap S^{q-1} \neq \emptyset, S^{q-1} \cap P^{\prime}$ is properly embedded in $P^{\prime} \backslash F$ (that is, $S^{q-1} \cap \partial\left(P^{\prime} \backslash F\right)=\partial\left(S^{q-1} \cap P^{\prime}\right)$ ).

We will show that there is an $m$-dimensional cube $B^{m}=B^{q} \times B^{m-q} \subset$ Int $M$ such that $B^{m}$ is PL-embedded in $M$, and $B^{q}=B^{q} \times O$ and $B^{m}$ have the following properties: $S^{q-1}=\partial B^{q}, \mathcal{P}$ restricted to $\partial B^{m} \backslash F$ and to $M \backslash\left(\right.$ Int $\left.B^{m} \cup F\right)$ are partitions, $F \cap B^{m}=\left(F \cap B^{q}\right) \times B^{m-q}, P^{\prime} \cap B^{m}=$ $\left(P^{\prime} \cap B^{q}\right) \times B^{m-q}$ for every $P^{\prime} \in \mathcal{P}$ and $P^{\prime} \backslash\left(F \cup \operatorname{Int} B^{m}\right)$ is a deformation retract of $P^{\prime} \backslash\left(F \cup \operatorname{Int} B^{q}\right)$ for every finite intersection $P^{\prime}$ of $\mathcal{P}$.

Moreover, if $\Phi: B_{\Phi}^{q} \rightarrow M$ is a map from a $q$-dimensional cube $B_{\Phi}^{q}$ into $M$ such that $\Phi$ restricted to $\partial B_{\Phi}^{q}$ is a PL-homeomorphism between $\partial B_{\Phi}^{q}$ and $S^{q-1}$, then $B^{q}$ can be chosen to be arbitrarily close to $\Phi\left(B_{\Phi}^{q}\right)$ in the sense that $B^{q}$ can be chosen to be the image of an arbitrarily close approximation of $\Phi$ by a PL-embedding which coincides with $\Phi$ on $\partial B_{\Phi}^{q}$.

Clearly, replacing $B^{m-q}$ by a smaller cube we ensure that $B^{m}$ will be arbitrarily close to $B^{q}$. Hence, if $S^{q-1}$ can be contracted to a point in an open subset $S^{q-1} \subset W$ of $M$, then we can assume that $B^{m} \subset W$.

Construction. The case $q=1$ and $m=3$ can be visualized directly and is left to the reader. Assume that $m \geq 4$ (in fact, we can always assume that $m \geq 4$ restricting ourselves to Nöbeling spaces modeled on manifolds of dimension $\geq 4$ ). Let $B^{q}$ be a $q$-dimensional ball PL-embedded in Int $M$ such that $S^{q-1}=\partial B^{q}$. Using an unknotting theorem for manifolds (see Theorem 10.3 of [6]) find a PL-embedded Euclidean space $\mathbb{R}^{m}=\mathbb{R}^{q} \times$ $\mathbb{R}^{m-q} \subset$ Int $M$ such that $B^{q} \subset \mathbb{R}^{q}$, where $\mathbb{R}^{q}$ and $\mathbb{R}^{m-q}$ are identified with the subsets $\mathbb{R}^{q} \times O \subset \mathbb{R}^{m}$ and $O \times \mathbb{R}^{m-q} \subset \mathbb{R}^{m}$ respectively.

Let $\Phi$ be as above. It is easy to see that $\Phi$ can be arbitrarily closely approximated by a PL-embedding into Int $M$. Then replacing $\Phi$ by such an approximation and setting $B^{q}=\Phi\left(B_{\Phi}^{q}\right)$ we can assume without loss of generality that $\Phi\left(B_{\Phi}^{q}\right) \subset \mathbb{R}^{m}$.

For a block bundle $\xi$ over a cell complex $X$, written $\xi / X$, denote by $\sigma_{\xi}$ the cells of $X$, by $\beta_{\xi}$ the blocks of the total space $E(\xi)$ and by $\left(\sigma_{\xi}, \beta_{\xi}\right)$ the pairs such that $\beta_{\xi}$ is the block over $\sigma_{\xi}$ (see [10]). We say that $\xi$ underlies $Y \subset E(\xi)$ if $Y$ is a union of blocks of $\xi$, and $\xi$ underlies a collection $\mathcal{Y}$ of subsets of $E(\xi)$ if $\xi$ underlies each $Y \in \mathcal{Y}$.

Let us say that a block bundle $\xi$ over $S^{q-1}$ is subordinated to $\mathcal{P}$ if $E(\xi)$ is a regular neighborhood of $S^{q-1}$ in $\mathbb{R}^{m}, E(\xi)$ does not intersect $F$ and $E(\xi)$ underlies $\mathcal{P}$ restricted to $E(\xi)$. 
A block bundle $\xi$ subordinated to $\mathcal{P}$ can be constructed as follows. Fix a triangulation $\mathcal{T}$ of $M$ which is the second barycentric subdivision of a triangulation that underlies $S^{q-1}, F$ and $\mathcal{P}$. By a simplicial neighborhood of $A \subset M$ in a subcomplex $X$ of $M$ we mean the union of the simplexes of $\mathcal{T}$ intersecting $A$ and contained in $X$. We may assume that $\mathcal{T}$ is chosen so that the simplicial neighborhood $G$ of $S^{q-1}$ in $M$ is contained in $\mathbb{R}^{m}$ and $G \cap F=\emptyset$. For every finite intersection $P^{\prime}$ of $\mathcal{P}$ that meets $S^{q-1}$ denote by $G_{P^{\prime}}$ the simplicial neighborhood of $P^{\prime} \cap S^{q-1}$ in $P^{\prime}$. Note that $G_{P^{\prime}}=G \cap P^{\prime}$, $G_{P^{\prime \prime}}=G_{P^{\prime}} \cap P^{\prime \prime}$ if $P^{\prime \prime} \subset P^{\prime}, G_{P^{\prime}}$ is a regular neighborhood of $P^{\prime} \cap S^{q-1}$ in $P^{\prime} \backslash F$ and $P^{\prime} \cap S^{q-1}$ is locally flat in $P^{\prime} \backslash F$ because $\operatorname{dim} P^{\prime}-\operatorname{dim} P^{\prime} \cap S^{q-1}=$ $m-(q-1) \geq q+2$.

For every finite intersection $P^{\prime}$ of $\mathcal{P}$ that meets $S^{q-1}$ we are going to construct by induction on $\operatorname{dim} P^{\prime} \cap S^{q-1}$ a block bundle $\xi_{P^{\prime}}$ over $P^{\prime} \cap S^{q-1}$ such that $E\left(\xi_{P^{\prime}}\right)=G_{P^{\prime}}$ and $\xi_{P^{\prime}} \mid P^{\prime \prime} \cap S^{q-1}=\xi_{P^{\prime \prime}}$ if $P^{\prime \prime} \subset P^{\prime}$. Obviously, such a block bundle exists if $\operatorname{dim} P^{\prime} \cap S^{q-1}=0$. Assume that for a finite intersection $P^{\prime}$ of $\mathcal{P}$ a block bundle $\xi_{P^{\prime \prime}}$ is already constructed for every finite intersection $P^{\prime \prime}$ of $\mathcal{P}$ such that $P^{\prime \prime} \subset P^{\prime}$ and $P^{\prime \prime} \neq P^{\prime}$. Then $\xi_{P^{\prime \prime}}$ defines the corresponding block bundle $\xi_{\partial P^{\prime}}$ over $\partial\left(P^{\prime} \cap S^{q-1}\right)$ and by Theorem 4.3 of [10] this block bundle extends to a block bundle $\xi_{P^{\prime}}$ over $P^{\prime}$ such that $E\left(\xi_{P^{\prime}}\right)=G_{P^{\prime}}$.

Thus we have constructed block bundles $\xi_{P^{\prime}}$ for every finite intersection $P^{\prime}$ of $\mathcal{P}$ that meets $S^{q-1}$, and these block bundles define the corresponding block bundle $\xi$ over $S^{q-1}$. Clearly, $\xi$ is subordinated to $\mathcal{P}$ and $E(\xi)=G$.

Let $B_{1}^{m-q}$ be a cube in $\mathbb{R}^{m-q}$. Fix $\varepsilon=1 / 2$ and for each cell $\sigma_{\xi}$ of $S^{q-1}$ define the pair $\left(\sigma_{\eta}, \beta_{\eta}\right)$ with $\sigma_{\eta}=\sigma_{\xi}$ and $\beta_{\eta}=\gamma_{\xi} \times B_{1}^{m-q}$ where $\gamma_{\xi}=$ $\left\{x: x=t s, 1-\varepsilon \leq t \leq 1+\varepsilon, s \in \sigma_{\xi}\right\} \subset \mathbb{R}^{q}$. Then the pairs $\left(\sigma_{\eta}, \beta_{\eta}\right)$ form a block bundle $\eta$ over $S^{q-1}$ such that $E(\eta)$ underlies a regular neighborhood of $S^{q-1}$ in $\mathbb{R}^{m}$. Hence by Theorem 4.4 of [10] there is a PL-homeomorphism of $\mathbb{R}^{m}$ realizing an isomorphism from $\eta$ to $\xi$.

Let $e: \mathbb{R}^{m} \rightarrow M$ be the composition of such a PL-homeomorphism with the original embedding of $\mathbb{R}^{m}$ in $M$. Then replacing the original embedding of $\mathbb{R}^{m}$ into $M$ by the embedding $e$ we may assume that $\xi$ coincides with $\eta$ (the meaning of such a replacement is that we leave $\mathbb{R}^{m}$ as the same subset of $M$ but change the representation of $\mathbb{R}^{m}$ as the product of $\mathbb{R}^{q}$ and $\mathbb{R}^{m-q}$ ). Thus we deduce that $\eta$ is subordinated to $\mathcal{P}$ and this is the only property of $\eta$ with respect to $\mathcal{P}$ that will be used in what follows.

Replace the map $\Phi$ by a close PL-embedding which coincides with $\Phi$ on $\partial B_{\Phi}^{q}$ and sends a small neighborhood of $\partial B_{\Phi}^{q}$ in $B_{\Phi}^{q}$ onto a neighborhood of $\partial B^{q}$ in $B^{q}$. Then there is a neighborhood $\Omega$ of $S^{q-1}$ in $\mathbb{R}^{m}$ such that $\Phi\left(B_{\Phi}^{q}\right) \cap \Omega=B^{q} \cap \Omega$. It is easy to see from the definition of $\eta$ that there is a PL-homeomorphism $\phi_{1}: \mathbb{R}^{m} \rightarrow \mathbb{R}^{m}$ such that $\phi_{1}$ does not move the points 
of $S^{q-1}, \phi_{1}\left(B^{q}\right)=B^{q}, \phi_{1}(E(\eta)) \subset E(\eta) \cap \Omega$ and for every block $\beta_{\eta}$ we have $\phi_{1}\left(\beta_{\eta}\right) \subset \beta_{\eta}$. Then the block bundle $\phi_{1}(\eta)$ is also subordinated to $\mathcal{P}$ and we can replace the embedding $e$ by $e \circ \phi_{1}$ and assume that $E(\eta) \subset \Omega$ and $\Phi\left(B_{\Phi}^{q}\right) \cap E(\eta)=B^{q} \cap E(\eta)$.

By an unknotting theorem for manifolds (see Theorem 10.1 of [6]) there is a PL-homeomorphism $\phi_{2}: \mathbb{R}^{m} \rightarrow \mathbb{R}^{m}$ such that $\phi_{2}$ does not move the points of $E(\eta)$ and $\Phi\left(B_{\Phi}^{q}\right)=\phi_{2}\left(B^{q}\right)$. Then replacing the embedding $e$ by $e \circ \phi_{2}$ we may assume that $\Phi\left(B_{\Phi}^{q}\right)=B^{q}$.

Fix a triangulation $\mathcal{T}_{\mathbb{R}}$ of $\mathbb{R}^{m}$ such that the simplexes of $\mathcal{T}_{\mathbb{R}}$ are linear in $\mathbb{R}^{m}$ and $\mathcal{T}_{\mathbb{R}}$ underlies $\mathcal{P} \mid \mathbb{R}^{m}, F \cap \mathbb{R}^{m}$ and $B_{1}^{q} \times B_{1}^{m-q}$ where $B_{1}^{q}=(1+\varepsilon) B^{q}$. Let $p: \mathbb{R}^{m}=\mathbb{R}^{q} \times \mathbb{R}^{m-q} \rightarrow \mathbb{R}^{m-q}$ be the projection and let $a \in \operatorname{Int} B_{1}^{m-q}$ be such that $a \notin p(\Delta)$ for every $\Delta \in \mathcal{T}_{\mathbb{R}}$ with $\operatorname{dim} p(\Delta)<m-q$ and $a \notin \partial p(\Delta)$ for every $\Delta \in \mathcal{T}_{\mathbb{R}}$ with $\operatorname{dim} p(\Delta)=m-q$. Take a cube $B_{2}^{m-q}$ such that $a+B_{2}^{m-q} \subset \operatorname{Int} B_{1}^{m-q}$ and $a+B_{2}^{m-q} \subset \operatorname{Int} p(\Delta)$ for every $\Delta \in \mathcal{T}_{\mathbb{R}}$ such that $\Delta \cap\left(B_{1}^{q} \times a\right) \neq \emptyset$. Then the pairs $\left(\sigma_{\tau}, \beta_{\tau}\right)$ with $\sigma_{\tau}=\left(B_{1}^{q} \times a\right) \cap \Delta$ and $\beta_{\tau}=\left(B_{1}^{q} \times\left(a+B_{2}^{m-q}\right)\right) \cap \Delta, \Delta \in \mathcal{T}_{\mathbb{R}}$, form a block bundle $\tau$ over $B_{1}^{q} \times a$, and we assume that $B_{2}^{m-q}$ is so small that $E(\tau)=B_{1}^{q} \times\left(a+B_{2}^{m-q}\right)$ and $E\left(\tau \mid \partial B_{1}^{q}\right)=\partial B_{1}^{q} \times\left(a+B_{2}^{m-q}\right)$.

By Theorem 1.1 of [10] every block bundle over a cube is trivial. Hence $\tau$ is isomorphic to a trivial block bundle $\delta$ over $B_{1}^{q}$, that is, there is a PL-homeomorphism $h: E(\delta) \rightarrow E(\tau)$ where $E(\delta)$ is the product $E(\delta)=$ $B_{1}^{q} \times B_{3}^{m-q} \subset \mathbb{R}^{m}$ of $B_{1}^{q}$ with a cube $B_{3}^{m-q}$ in $\mathbb{R}^{m-q}$ such that $h(x)=(x, a)$ for every $x \in B_{1}^{q},\left(\sigma_{\delta}, \beta_{\delta}\right)$ is a pair of $\delta$ if and only if $\left(h\left(\sigma_{\delta}\right), h\left(\beta_{\delta}\right)\right)$ is a pair of $\tau$ and, for every pair $\left(\sigma_{\delta}, \beta_{\delta}\right)$ of $\delta, \beta_{\delta}=\sigma_{\delta} \times B_{3}^{m-q}$.

Identify $B_{1}^{q} \times B_{3}^{m-q}$ with $h\left(B_{1}^{q} \times B_{3}^{m-q}\right)$ (that is, re-embed $B_{1}^{q} \times B_{3}^{m-q}$ according to $h)$ and let $B^{m-q}$ be a cube lying in $\operatorname{Int} B_{3}^{m-q}$. Then from the construction it follows that

(*) $P^{\prime} \cap\left(B_{1}^{q} \times \operatorname{Int} B_{3}^{m-q}\right)=\left(P^{\prime} \cap B_{1}^{q}\right) \times \operatorname{Int} B_{3}^{m-q}$ for every $P^{\prime} \in \mathcal{P}$ and $F \cap\left(B_{1}^{q} \times \operatorname{Int} B_{3}^{m-q}\right)=\left(F \cap B_{1}^{q}\right) \times \operatorname{Int} B_{3}^{m-q}$.

From the construction it also follows that $\mathcal{P}$ restricted to $\operatorname{Int} B_{1}^{q} \backslash \operatorname{Int} B^{q}$, to $\partial B^{q}$ and to $B^{q} \backslash F$ are partitions. Hence by $(*)$ and $2.2, \mathcal{P}$ restricted to $\left(\operatorname{Int} B_{1}^{q} \backslash \operatorname{Int} B^{q}\right) \times \operatorname{Int} B_{3}^{m-q}$, to $\left(B^{q} \backslash F\right) \times\left(\operatorname{Int} B_{3}^{m-q} \backslash \operatorname{Int} B^{m-q}\right)$, to $\partial B^{q} \times\left(\right.$ Int $\left.B_{3}^{m-q} \backslash \operatorname{Int} B^{m-q}\right)$, to $\partial B^{q} \times \partial B^{m-q}, \partial B^{q} \times B^{m-q}$ and to $\left(B^{q} \backslash F\right) \times \partial B^{m-q}$ are partitions. Then, once again by $2.2, \mathcal{P}$ restricted to $\operatorname{Int}\left(B_{1}^{q} \times B_{3}^{m-q}\right) \backslash\left(F \cup\left(\operatorname{Int}\left(B^{q} \times B^{m-q}\right)\right)\right)$ and to $\partial\left(B^{q} \times B^{m-q}\right) \backslash F$ are partitions.

Define $B^{m}=B^{q} \times B^{m-q}$. Thus we see that

(**) $\mathcal{P}$ restricted to $M \backslash\left(F \cup \operatorname{Int} B^{m}\right)$ and to $\partial B^{m} \backslash F$ are partitions.

Let $P^{\prime}$ be a finite intersection of $\mathcal{P}$. Let us show that 
$(* * *) P^{\prime} \backslash\left(F \cup \operatorname{Int} B^{m}\right)$ is a deformation retract of $P^{\prime} \backslash\left(F \cup \operatorname{Int} B^{q}\right)$.

Note the following general fact: for a triangulated space $A_{1}$, a subcomplex $A_{2}$ of $A_{1}$ and a cube $B$, the space $\left(A_{2} \times B\right) \cup\left(A_{1} \times \partial B\right)$ is a strong deformation retract of $\left(A_{2} \times B\right) \cup\left(A_{1} \times(B \backslash O)\right)$. Then for $A_{1}=\left(P^{\prime} \cap B^{q}\right) \backslash F$ and $A_{2}=P^{\prime} \cap \partial B^{q}$ we deduce by $(*)$ that $B^{m} \cap\left(P^{\prime} \backslash\left(F \cup \operatorname{Int} B^{m}\right)\right)=\left(A_{2} \times\right.$ $\left.B^{m-q}\right) \cup\left(A_{1} \times \partial B^{m-q}\right)$ and $B^{m} \cap\left(P^{\prime} \backslash\left(F \cup \operatorname{Int} B^{q}\right)\right)=\left(A_{2} \times B^{m-q}\right) \cup\left(A_{1} \times\right.$ $\left.\left(B^{m-q} \backslash O\right)\right)$. Thus $B^{m} \cap\left(P^{\prime} \backslash\left(F \cup \operatorname{Int} B^{m}\right)\right)$ is a strong deformation retract of $B^{m} \cap\left(P^{\prime} \backslash\left(F \cup \operatorname{Int} B^{q}\right)\right)$ and hence $(* * *)$ holds.

Let us compare the embeddings of $B^{q}$ under $h$ and $e$. For this purpose we assume that $\mathbb{R}^{m} \subset M$ is identified with $\mathbb{R}^{q} \times \mathbb{R}^{m-q}$ according to the embedding $e$ and we disregard the identification induced by $h$. Then $h(x)=$ $(x, a)$ for $x \in B^{q}$. One can easily observe that there is a PL-homeomorphism $\psi: M \rightarrow M$ such that $\psi \circ h$ does not move the points of $S^{q-1}, \psi$ does not move the points outside $\operatorname{Int}(E(\eta))$ and $\psi\left(P^{\prime}\right)=P^{\prime}$ for every $P^{\prime} \in \mathcal{P}$. Moreover, choosing the point $a$ arbitrarily close to $O$ we may choose $\psi$ to be arbitrarily close to the identity map of $M$. Note that since $\psi$ does not move the points outside $\operatorname{Int}(E(\eta))$, we have $\psi(F)=F$. Now we can replace $\Phi$ by $\psi \circ h \circ \Phi$ and $h$ by $\psi \circ h$, and after identifying $B_{1}^{q} \times B_{3}^{m-q}$ with its image under the new embedding $h$, we find that $\Phi\left(B_{\Phi}^{q}\right)=B^{q}$ and the properties $(*)-(* * *)$ are preserved.

Note that $(*)$ trivially implies that $F \cap B^{m}=\left(F \cap B^{q}\right) \times B^{m-q}$ and $P^{\prime} \cap B^{m}=\left(P^{\prime} \cap B^{q}\right) \times B^{m-q}$ for every $P^{\prime} \in \mathcal{P}$.

Thus we have replaced the original map $\Phi$ by an arbitrarily close PLembedding which coincides with $\Phi$ on $\partial B_{\Phi}^{q}$ and we have found a PL-embedding of $B^{m}=B^{q} \times B^{m-q} \subset \operatorname{Int} M$ such that $B^{q}=\Phi\left(B_{\Phi}^{q}\right)$ and $B^{m}$ has the required properties.

\subsection{Digging holes for absorbing simplexes}

Summary. Here we present a construction used in 2.6. Let $M$ be a triangulated $(q-1)$-connected $m$-dimensional manifold with $m \geq 2 q+1$ and let $l=m-q+1$. Assume that $F$ is a PL-subcomplex of $M$ lying in Int $M$ such that $U=M \backslash F$ is $l$-co-connected $(=(q-1)$-connected) and $\operatorname{dim} F \leq m-q$, and assume that $\mathcal{P}$ is a decomposition of $M$ such that $\mathcal{P}$ is an $l$-co-connected partition on $U$.

Fix a triangulation $\mathcal{T}_{0}$ of $M$ which underlies $F$ and the elements of $\mathcal{P}$ and let the triangulation $\mathcal{T}$ be the second barycentric subdivision of $\mathcal{T}_{0}$. Let $\mathcal{T}_{F}$ be the collection of all $(m-q)$-dimensional simplexes of $\mathcal{T}$ lying in $F$ and let $S_{\Delta}$ and $\Delta * S_{\Delta}$ be the link of $\Delta$ and the join of $\Delta$ with $S_{\Delta}$ respectively. Recall that

$$
\begin{aligned}
S_{\Delta} & =\bigcup\left\{\Delta^{\prime} \in \mathcal{T}: \Delta^{\prime} \cap \Delta=\emptyset \text { and there is } \Delta^{\prime \prime} \in \mathcal{T} \text { with } \Delta, \Delta^{\prime} \subset \Delta^{\prime \prime}\right\}, \\
\Delta * S_{\Delta} & =\bigcup\left\{\Delta^{\prime} \in \mathcal{T}: \Delta \subset \Delta^{\prime}\right\} .
\end{aligned}
$$


Define $Q$ as the union of $\operatorname{Int}\left(\Delta * S_{\Delta}\right)$ for all $\Delta$ in $\mathcal{T}_{F}$ and $N=M \backslash(Q \cup F)$. Let $\Delta \in \mathcal{T}_{F}$ and let an open subset $V_{\Delta}$ of $N$ be such that $S^{q-1}=S_{\Delta} \subset V_{\Delta}$ and $S^{q-1}$ is contractible in $V_{\Delta}$.

We will show that there are an element $P_{\Delta} \in \mathcal{P}$ and a PL-embedding of a cube $B^{m}=B^{q} \times B^{m-q}$ into Int $M$ such that $B^{m} \subset\left(\Delta * S_{\Delta}\right) \cup V_{\Delta}$, $\Delta=O \times B^{m-q}=F \cap B^{m}, P \cap U \cap B^{m}=\left(P \cap U \cap B^{q}\right) \times B^{m-q}$ for every $P \in \mathcal{P}, \partial B^{q} \times B^{m-q} \subset \operatorname{Int}\left(P_{\Delta} \cap U\right), \mathcal{P}$ restricted to $\left(M \backslash \operatorname{Int} B^{m}\right) \cap U$ and to $\partial B^{m} \cap U$ are partitions, and $\left(P \backslash \operatorname{Int} B^{m}\right) \cap U$ is a deformation retract of $\left(P \backslash \operatorname{Int} B^{q}\right) \cap U$ for every finite intersection $P$ of $\mathcal{P}$.

Construction. Let us repeat a few simple observations noted in 4.5 which immediately follow from the fact that $\mathcal{T}$ is the second barycentric subdivision of a triangulation which underlies $F$ and the elements of $\mathcal{P}$. Namely, we note that for every $\Delta \in \mathcal{T}_{F}$ the link $S_{\Delta}$ of $\Delta$ is a $(q-1)$-dimensional sphere lying in Int $M$, and for different simplexes $\Delta_{1}$ and $\Delta_{2}$ in $\mathcal{T}_{F}$ the joins $\Delta_{1} * S_{\Delta_{1}}$ and $\Delta_{2} * S_{\Delta_{2}}$ do not intersect on $U$. We also note that for every subset $H$ which is the union of a finite intersection of $\mathcal{P}$ such that $H \cap U \neq \emptyset$, and for every $\Delta \in \mathcal{T}_{F}$, we know that $(H \cap U) \backslash \operatorname{Int}\left(\Delta * S_{\Delta}\right)$ is a deformation retract of $H \cap U$ and hence $H \cap N$ is a deformation retract of $H \cap U$. Then $N$ is an l-co-connected manifold (since $N$ is a deformation retract of $U$ ) and $\mathcal{P} \mid N$ is an $l$-co-connected partition (since $\mathcal{P} \mid U$ is l-co-connected).

Consider $M$ as embedded in a Hilbert space by an embedding which is linear on every simplex of $\mathcal{T}_{0}$.

Fix $\Delta \in \mathcal{T}_{F}$ and let $S^{q-1}=S_{\Delta}$ be the link of $\Delta$ with respect to $\mathcal{T}$, $S^{m-1}=\partial\left(\Delta * S_{\Delta}\right)$ and $b$ be the barycenter of $\Delta$.

Let us show that $\mathcal{P}$ restricted to $N$, to $S^{m-1} \backslash \partial \Delta$ and to $\left(\Delta * S_{\Delta}\right) \backslash \Delta$ are partitions. For a point $x \in S^{m-1} \backslash \partial \Delta$ choose an $\varepsilon>0$ and a neighborhood $G$ of $x$ in $S^{m-1} \backslash \partial \Delta$ such that the map $(z, t) \mapsto z+t b, z \in G, t \in(-\varepsilon, \varepsilon)$, embeds $G \times(-\varepsilon, \varepsilon)$ into $M \backslash F$ and for every simplex $\Delta^{\prime}$ of $\mathcal{T}_{0}$ that intersects $G \times(-\varepsilon, \varepsilon)$ we have $\Delta^{\prime} \cap(G \times(-\varepsilon, \varepsilon))=\left(\Delta^{\prime} \cap G\right) \times(-\varepsilon, \varepsilon)$. Then for every $P \in \mathcal{P}$ that intersects $G \times(-\varepsilon, \varepsilon)$ we have $P \cap(G \times(-\varepsilon, \varepsilon))=(P \cap G) \times(-\varepsilon, \varepsilon)$. Therefore, by $2.2, \mathcal{P}$ restricted to $G$, to $G \times(-\varepsilon, 0]$ and to $G \times[0, \varepsilon)$ are partitions and hence $\mathcal{P}$ restricted to $S^{m-1} \backslash \partial \Delta$, to $\left(\Delta * S_{\Delta}\right) \backslash \Delta$ and to $N$ are partitions as well.

Denote by $\mathcal{T}_{\Delta}$ the collection of the simplexes of $\mathcal{T}$ intersecting $S^{q-1}$ and containing $\Delta$. Let $\mathcal{T}^{\prime}$ be the second barycentric subdivision of $\mathcal{T}$. For every $\Delta^{\prime} \in \mathcal{T}_{\Delta}$ denote by $G_{\Delta^{\prime}}$ the simplicial neighborhood of $\Delta^{\prime} \cap S^{q-1}$ in $\Delta^{\prime} \cap S^{m-1}$ with respect to $\mathcal{T}^{\prime}$. In a way similar to constructing $\xi_{P^{\prime}}$ in 5.2 we construct for every $\Delta^{\prime} \in \mathcal{T}_{\Delta}$ a block bundle $\xi_{\Delta^{\prime}}$ over $\Delta^{\prime} \cap S^{q-1}$ such that $E\left(\xi_{\Delta^{\prime}}\right)=G_{\Delta^{\prime}}$ and $\xi_{\Delta^{\prime}} \mid\left(\Delta^{\prime \prime} \cap S^{m-1}\right)=\xi_{\Delta^{\prime \prime}}$ if $\Delta^{\prime \prime} \subset \Delta^{\prime}$. Then the block bundles $\xi_{\Delta^{\prime}}$ define the corresponding block bundle $\xi$ over $S^{q-1}$ with $E(\xi)$ 
being the simplicial neighborhood of $S^{q-1}$ in $S^{m-1}$ with respect to $\mathcal{T}^{\prime}$. Note that $E(\xi)$ is a regular neighborhood of $S^{q-1}$ in $\partial N$.

Extend the embedding of $S^{q-1}$ in $N$ to a PL-embedding of a $q$-dimensional cube $B_{1}^{q}$ into $N$ such that $S^{q-1}=\partial B_{1}^{q}$ and $B_{1}^{q} \cap \partial N=S^{q-1}$, and note that the embedding of $B_{1}^{q}$ is locally flat because $m \geq 2 q+1$. Extend the regular neighborhood $E(\xi)$ of $S^{q-1}$ in $\partial N$ to a regular neighborhood of $B_{1}^{q}$ in $N$, and by Theorem 4.3 of [10] represent this neighborhood as the total space $E(\nu)$ of a block bundle $\nu$ over $B_{1}^{q}$ such that $\nu \mid S^{q-1}=\xi$. Since $\nu$ is a trivial block bundle, $E(\nu)$ is PL-homeomorphic to the product $E(\nu)=B_{1}^{q} \times B_{1}^{m-q}$ of $B_{1}^{q}$ with an $(m-q)$-dimensional cube $B_{1}^{m-q}$ such that for each pair $\left(\sigma_{\nu}, \beta_{\nu}\right)$ of $\nu, \beta_{\nu}=\sigma_{\nu} \times B_{1}^{m-q}$. Fix a triangulation $\mathcal{T}_{\nu}$ of $E(\nu)$ which underlies $\mathcal{T}$ restricted to $E(\nu)$. In a way similar to constructing the block bundle $\tau$ in 5.2 choose a point $a$ in $B_{1}^{m-q}$ and a cube $B_{2}^{m-q}$ such that $a+B_{2}^{m-q} \subset \operatorname{Int} B_{1}^{m-q}$ and the pairs $\left(\sigma_{\tau}, \beta_{\tau}\right)$ defined by $\sigma_{\tau}=\Delta^{\prime} \cap\left(B_{1}^{q} \times a\right)$, $\beta_{\tau}=\Delta^{\prime} \cap\left(B_{1}^{q} \times\left(a+B_{2}^{m-q}\right)\right), \Delta^{\prime} \in \mathcal{T}_{\nu}$, form a block bundle $\tau$ over $B_{1}^{q} \times a$ with $E(\tau)=B_{1}^{q} \times\left(a+B_{2}^{m-q}\right)$ and $E\left(\tau \mid \partial\left(B_{1}^{q} \times a\right)\right)=E(\tau) \cap\left(\partial B_{1}^{q} \times\left(a+B_{2}^{m-q}\right)\right)$ $\left(=E(\tau) \cap S^{m-1}\right)$. Then the block bundle $\tau$ underlies $\mathcal{P}$ and $\mathcal{T}_{\Delta}$ restricted to $E(\tau)$.

Note that since the block bundle $\nu$ is trivial, the block bundle $\xi$ is trivial as well and $E(\xi)=\partial B_{1}^{q} \times B_{1}^{m-q}$. Then it is obvious that the projection $\partial B_{1}^{q} \times a \rightarrow \partial B_{1}^{q}$ extends to a PL-homeomorphism $h: E(\xi) \rightarrow E(\xi)$ such that $h(x)=x$ for every $x \in \partial E(\xi), h\left(\beta_{\xi}\right)=\beta_{\xi}$ for every block $\beta_{\xi}$ of $\xi$ and there is a PL-isotopy $H: E(\xi) \times[0,1] \rightarrow E(\xi) \times[0,1]$ relative to $\partial E(\xi)$ so that $H_{0}=h$. Recall that the barycenter of $\Delta$ is denoted by $b$. Then, since $\partial \Delta \cap E(\xi)=\emptyset$, there is $\varepsilon>0$ such that for every $x \in E(\xi), P \in \mathcal{P}$ and $t \in[0,1]$, the point $b+(1+t \varepsilon)(x-b)$ belongs to $P$ if and only if $x$ belongs to $P$. Embed $E(\xi) \times[0,1]$ into $N$ by sending $(x, t)$ to $b+(1+t \varepsilon)(x-b)$ and define the PL-homeomorphism $g: N \rightarrow N$ such that $g$ coincides with $H$ on $E(\xi) \times[0,1]$ and $g$ does not move the points outside $E(\xi) \times[0,1]$. From the properties described above it is clear that $g\left(\partial\left(B_{1}^{q} \times a\right)\right)=\partial B_{1}^{q}$ and the block bundle $g(\tau)$ underlies $\mathcal{P}$ and $\mathcal{T}_{\Delta}$ restricted to $E(g(\tau))$. Define $B_{2}^{q}=g\left(B_{1}^{q} \times a\right)$ and $\theta=g(\tau)$.

Thus we get a block bundle $\theta$ over a cube $B_{2}^{q}$ such that $S^{q-1}=\partial B_{2}^{q}=$ $\partial N \cap B_{2}^{q}, \theta$ underlies $\mathcal{P}$ and $\mathcal{T}_{\Delta}$ restricted to $E(\theta)$, and $E\left(\theta \mid \partial B_{2}^{q}\right)=S^{m-1} \cap$ $E(\theta)$. Then, since $\theta$ is trivial, $E(\theta)$ is PL-homeomorphic to $B_{2}^{q} \times B_{3}^{m-q}$ such that $\partial N \cap\left(B_{2}^{q} \times B_{3}^{m-q}\right)=\partial B_{2}^{q} \times B_{3}^{m-q} \subset S^{m-1}$ and for each pair $\left(\sigma_{\theta}, \beta_{\theta}\right)$ of $\theta, \beta_{\theta}=\sigma_{\theta} \times B_{3}^{m-q}$. Take a cube $B_{4}^{m-q}$ such that $B_{4}^{m-q} \subset \operatorname{Int} B_{3}^{m-q}$ and define the block bundle $\varrho$ over $B_{2}^{q}$ by restricting the blocks of $\theta$ to $E(\varrho)=B_{2}^{q} \times B_{4}^{m-q}$. Using a reasoning similar to the one applied for proving the property $(* *)$ in 5.2 we can show that $\mathcal{P}$ restricted $N \backslash\left(B_{2}^{q} \times \operatorname{Int} B_{4}^{m-q}\right)$ 
and to $\partial\left(\left(\Delta * S_{\Delta}\right) \cup\left(B_{2}^{q} \times B_{4}^{m-q}\right)\right) \backslash \partial \Delta$ are partitions. It is clear that $E(\varrho) \subset \operatorname{Int} M$.

Take a pair $\left(\sigma_{\varrho}^{\Delta}, \beta_{\varrho}^{\Delta}\right)$ of $\varrho$ such that $\operatorname{dim} \beta_{\varrho}^{\Delta}=m$ and let $P_{\Delta} \in \mathcal{P}$ be such that $\beta_{\varrho}^{\Delta} \subset P_{\Delta}$. Since $\varrho$ is a trivial block bundle we can replace $\varrho$ by another block bundle subdividing the cells of $B_{2}^{q}$ into smaller cells and defining the corresponding blocks using the product structure. This way we may assume that the cells of $B_{2}^{q}$ are so small that the pair $\left(\sigma_{\varrho}^{\Delta}, \beta_{\varrho}^{\Delta}\right)$ can be chosen so that $\beta_{\varrho}^{\Delta} \subset \operatorname{Int}\left(P_{\Delta} \cap N\right)$.

Let the cube $B_{3}^{q} \subset \Delta * S_{\Delta}$ be the join $B_{3}^{q}=b * S_{\Delta}$. Define a block bundle $\psi$ over $B_{3}^{q}$ as follows: the pairs $\left(\sigma_{\varrho}, \beta_{\varrho}\right)$ of $\varrho$ with $\sigma_{\varrho} \subset S^{q-1}$ are the pairs of $\psi,(b, \Delta)$ is a pair of $\psi$ and the rest of the pairs $\left(\sigma_{\psi}, \beta_{\psi}\right)$ of $\psi$ are of the form $\sigma_{\psi}=\Delta^{\prime} \cap B_{3}^{q}, \beta_{\psi}=\Delta^{\prime}, \Delta^{\prime} \in \mathcal{T}_{\Delta}$. One can easily check that $\psi$ is indeed a block bundle and $E(\psi)=\Delta * S_{\Delta}$. Since $\psi$ and $\varrho$ coincide over $S^{q-1}$, they form the corresponding block bundle $\phi$ over the sphere $B_{3}^{q} \cup B_{2}^{q}$. Note that $B^{q}=\left(B_{2}^{q} \cup B_{3}^{q}\right) \backslash \operatorname{Int} \sigma_{\varrho}^{\Delta}$ is a $q$-dimensional cube. Define the block bundle $\eta$ as $\phi$ restricted to $B^{q}$. Then $(b, \Delta)$ is a pair of $\eta, b \in \operatorname{Int} B^{q}, \partial B^{q}=\partial \sigma_{\varrho}^{\Delta}$, the intersections of the blocks of $\eta$ with $U=M \backslash F$ underlie $\mathcal{P}$ restricted to $E(\eta) \cap U, E(\eta) \cap F=\Delta$ and hence $\eta$ underlies $F$ restricted to $E(\eta)$.

Thus assuming that the center $O$ of $B^{q}$ is located at $b$ we can represent $E(\eta)$ as the product $B^{m}=B^{q} \times B^{m-q}$ such that $\Delta=0 \times B^{m-q}=F \cap B^{m}$, $\partial B^{q} \times B^{m-q} \subset \operatorname{Int}\left(P_{\Delta} \cap U\right)$ and $P \cap U \cap B^{m}=\left(P \cap U \cap B^{q}\right) \times B^{m-q}$ for every $P \in \mathcal{P}$ that meets $B^{m} \cap U$. By a reasoning similar to the one applied in the proof of $(* * *)$ in 5.2 these properties imply that for every finite intersection $P$ of $\mathcal{P}, P \backslash\left(F \cup \operatorname{Int} B^{m}\right)$ is a deformation retract of $P \backslash\left(F \cup \operatorname{Int} B^{q}\right)$. Since $B^{m}=\left(\Delta * S_{\Delta}\right) \cup\left(\left(B_{2}^{q} \backslash \operatorname{Int} \sigma_{\varrho}^{\Delta}\right) \times B_{4}^{m-q}\right)$ and $\beta_{\varrho}^{\Delta}=\sigma_{\varrho}^{\Delta} \times B_{4}^{m-q}$ is contained in $\operatorname{Int}\left(P_{\Delta} \cap U\right)$, we derive from the properties of $\varrho$ that $\mathcal{P}$ restricted to $M \backslash\left(F \cup \operatorname{Int} B^{m}\right)$ and to $\partial B^{m} \backslash F$ are partitions and $B^{m} \subset \operatorname{Int} M$.

Note that if $S^{q-1}$ can be contracted to a point in an open subset $V_{\Delta}$ of $N$ then the construction can be carried out so that $B^{m}$ is contained in $\left(\Delta * S_{\Delta}\right) \cup V_{\Delta}$.

\section{References}

[1] S. Ageev, Axiomatic method of partitions in the theory of Nöbeling spaces. I. Improvement of partition connectivity, Mat. Sb. 198 (2007), no. 3, 3-50 (in Russian); English transl.: Sb. Math. 198 (2007), 299-342.

[2] - , Axiomatic method of partitions in the theory of Nöbeling spaces. II. Unknotting theorem, Mat. Sb. 198 (2007), no. 5, 3-32 (in Russian); English transl.: Sb. Math. 198 (2007), 597-625.

[3] - Axiomatic method of partitions in the theory of Nöbeling spaces. III, Consistency of the axiom system, Mat. Sb. 198 (2007), no. 7, 3-30 (in Russian); English transl.: Sb. Math. 198 (2007), 909-934. 
[4] M. Bestvina, Characterizing k-dimensional universal Menger compacta, Mem. Amer. Math. Soc. 71 (1988), no. 380.

[5] A. Chigogidze, Inverse Spectra, North-Holland Math. Library 53, North-Holland, Amsterdam, 1996.

[6] J. F. P. Hudson, Piecewise Linear Topology, Benjamin, New York, 1969.

[7] K. Kawamura, M. Levin and E. D. Tymchatyn, A characterization of 1-dimensional Nöbeling spaces, Topology Proc. 22 (1997), 155-174.

[8] M. Levin, Characterizing Nöbeling spaces, preprint, 2006, arXiv:math.GT/0602361.

[9] A. Nagórko, Characterization and topological rigidity of Nöbeling manifolds, PhD thesis, 2006, arXiv:math.GT/0602574.

[10] C. P. Rourke and B. J. Sanderson, Block bundles. I, Ann. of Math. (2) 87 (1968), $1-28$.

[11] - - - Introduction to Piecewise-Linear Topology, Ergeb. Math. Grenzgeb. 69, Springer, New York, 1972.

Department of Mathematics

Ben Gurion University of the Negev

P.O. Box 653

Be'er Sheva 84105, Israel

E-mail: mlevine@math.bgu.ac.il

Received 31 October 2005;

in revised form 4 December 2007 and 1 July 2008 